\title{
Progress Toward the Enantioselective Synthesis of Curcusones A-D via a Divinylcyclopropane Rearrangement Strategy
}

Austin C. Wright, Chung Whan Lee, and Brian M. Stoltz

The Warren and Katharine Schlinger Laboratory for Chemistry and Chemical Engineering, Division of Chemistry and Chemical Engineering, California Institute of Technology, MC 101-20, Pasadena, California 91125, United States

\section{Table of Contents}

\section{Experimental Section}

\section{References}

\section{NMR and IR spectra of Unknown Compounds}




\section{Experimental Section}

\section{Materials and Methods}

Unless stated otherwise, reactions were performed under an argon or nitrogen atmosphere using dry, deoxygenated solvents (distilled or passed over a column of activated alumina). ${ }^{1} \mathrm{Et}_{3} \mathrm{~N}$, $i$ - $\mathrm{Pr}_{2} \mathrm{NEt}, i$ - $\mathrm{Pr}_{2} \mathrm{NH}$, pyridine, and $i$-PrOH were distilled from calcium hydride immediately prior to use. Commercially obtained reagents were used as received unless otherwise stated. $p$-ABSA, ${ }^{2}$ $\mathrm{Cu}(\mathrm{TBSal})_{2},{ }^{3}$ and $\mathrm{MoCl}_{3}(\mathrm{THF})_{2}{ }^{4}$ were prepared by known methods. Reactions were heated in an oil bath, and the temperatures were controlled by an IKAmag temperature modulator. Thin-layer chromatography (TLC) was performed using E. Merck silica gel 60 F254 precoated plates (0.25 $\mathrm{mm}$ ) and visualized by UV fluorescence quenching, or potassium permanganate, iodine, or anisaldehyde staining. SiliaFlash P60 Academic Silica gel (particle size 0.040-0.063 mm) was used for flash chromatography. ${ }^{1} \mathrm{H}$ and ${ }^{13} \mathrm{C}$ NMR spectra were recorded on a Varian Inova 600 (600 MHz and $151 \mathrm{MHz}$ respectively), Varian Inova 500 (at $500 \mathrm{MHz}$ and $126 \mathrm{MHz}$ respectively), Bruker AV III HD spectrometer equipped with a Prodigy liquid nitrogen temperature cryoprobe (400 $\mathrm{MHz}$ and $101 \mathrm{MHz}$, respectively) and are reported relative to $\mathrm{CHCl}_{3}(\delta 7.26 \& 77.16$ respectively), $\mathrm{C}_{6} \mathrm{H}_{6}\left(\delta 7.16 \& 128.06\right.$ respectively), and $\mathrm{CH}_{2} \mathrm{Cl}_{2}(\delta 5.32 \& 53.84$ respectively). Data for ${ }^{1} \mathrm{H}$ NMR spectra are reported as follows: chemical shift ( $\left.\delta \mathrm{ppm}\right)$ (multiplicity, coupling constant $(\mathrm{Hz})$, integration). IR spectra were recorded on a Perkin Elmer Paragon 1000 Spectrometer and are reported in frequency of absorption $\left(\mathrm{cm}^{-1}\right)$. HRMS were acquired from the Caltech Mass Spectral Facility using a JEOL JMS-600H High Resolution Mass Spectrometer in fast atom bombardment $(\mathrm{FAB}+)$ or electron ionization $(\mathrm{EI}+)$ mode or using an Agilent 6200 Series TOF with an Agilent G1978A Multimode source in electrospray ionization (ESI), atmospheric pressure 
chemical ionization (APCI) or mixed (MM) ionization mode. Optical rotations were measured on a Jasco P-2000 polarimeter using a $100 \mathrm{~mm}$ path length cell at $589 \mathrm{~nm}$. 


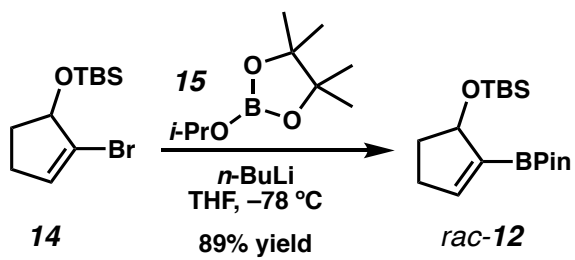

tert-Butyldimethyl((2-(4,4,5,5-tetramethyl-1,3,2-dioxaborolan-2-yl)cyclopent-2-en-1-

yl)oxy)silane (rac-12): To a flame-dried round-bottom flask with a magnetic stir bar were added bromide 14 (440 mg, $1.59 \mathrm{mmol})$ and THF (6 mL). The flask was cooled to $-78^{\circ} \mathrm{C}$ and stirred for 10 min. $n$-BuLi solution (2.1 $\mathrm{M}$ in hexanes, $0.95 \mathrm{~mL}, 2.00 \mathrm{mmol}$ ) was added dropwise. The reaction mixture was stirred at $-78{ }^{\circ} \mathrm{C}$ for $30 \mathrm{~min}$ then isopropyl pinacolyl borate $(\mathbf{1 5}, 0.40 \mathrm{~mL}$, $1.96 \mathrm{mmol}$ ) was added. The reaction mixture was stirred at $-78^{\circ} \mathrm{C}$ for $30 \mathrm{~min}$ then quenched with $\mathrm{HCl}$ solution $\left(2 \mathrm{~N}\right.$ in $\mathrm{Et}_{2} \mathrm{O}, 1.0 \mathrm{~mL}, 2.00 \mathrm{mmol}$ ). Following addition, the reaction mixture was diluted with $\mathrm{Et}_{2} \mathrm{O}(10 \mathrm{~mL})$ and warmed up to $23{ }^{\circ} \mathrm{C}$. The reaction mixture was filtered and concentrated under reduced pressure. The residue was purified by flash column chromatography (20:1 hexanes, EtOAc) to afford vinylboronate $r a c-12$ as a colorless oil (460 mg, $1.42 \mathrm{mmol}, 89 \%$ yield); $\mathrm{R}_{f}=0.60\left(20: 1\right.$ hexanes, EtOAc); ${ }^{1} \mathrm{H}$ NMR $\left(500 \mathrm{MHz}, \mathrm{CDCl}_{3}\right) \delta 6.62(\mathrm{td}, J=2.4,1.0 \mathrm{~Hz}$, 1H), 5.00 (dddt, $J=6.1,3.9,2.1,1.1 \mathrm{~Hz}, 1 \mathrm{H}), 2.56(\mathrm{dddt}, J=17.8,8.9,4.6,2.3 \mathrm{~Hz}, 1 \mathrm{H}), 2.34-2.20$ (m, 1H), 2.20-2.08 (m, 1H), 1.75-1.65 (m, 1H), $1.25(\mathrm{~d}, J=1.6 \mathrm{~Hz}, 12 \mathrm{H}), 0.89(\mathrm{~s}, 9 \mathrm{H}), 0.11(\mathrm{~s}$, $6 \mathrm{H}) ;{ }^{13} \mathrm{C} \mathrm{NMR}\left(126 \mathrm{MHz}, \mathrm{CDCl}_{3}\right) \delta 149.3,83.1,80.0,34.7,33.0,26.1,25.1,25.0,18.5,14.1,-4.6$; IR (Neat Film, NaCl) 3040, 2978, 2929, 2856, 2708, 1622, 1472, 1409, 1372, 1318, 1249, 1214, 1146, 1060, 1005, 964, 952, 936, 875, $855 \mathrm{~cm}^{-1}$; HRMS (FAB+) $\mathrm{m} / z$ calc'd for $\mathrm{C}_{17} \mathrm{H}_{32} \mathrm{SiO}_{3} \mathrm{~B}$ $\left[\mathrm{M}+\mathrm{H}-\mathrm{H}_{2}\right]^{+:}$: 323.2214, found 323.2222. 


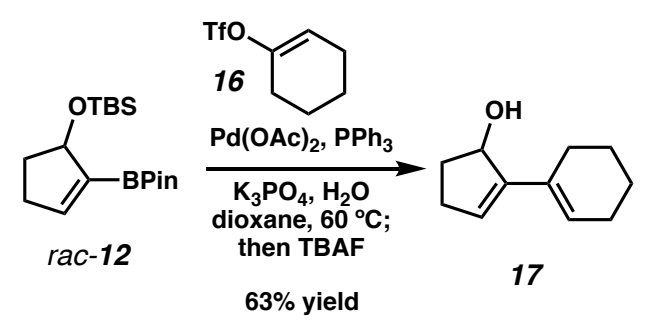

2-(Cyclohex-1-en-1-yl)cyclopent-2-en-1-ol (17): To a flame-dried round-bottom flask equipped with a magnetic stir bar were added boronate rac-12 (2.25 g, $6.94 \mathrm{mmol})$, triflate 16 (1.71 g, $7.43 \mathrm{mmol})$, palladium acetate $(70 \mathrm{mg}, 0.311 \mathrm{mmol})$, triphenylphosphine $(180 \mathrm{mg}, 0.686$ $\mathrm{mmol})$, and tribasic potassium phosphate $(4.43 \mathrm{~g}, 20.87 \mathrm{mmol})$. The mixture was evacuated and back filled with argon (x3). The mixture was dissolved in dioxane (35 mL) and water (3.5 mL). The reaction was immersed in a $60{ }^{\circ} \mathrm{C}$ oil bath. After $9 \mathrm{~h}$ of stirring, the reaction was cooled to ambient temperature, diluted with EtOAc $(10 \mathrm{~mL})$, and quenched with saturated $\mathrm{NH}_{4} \mathrm{Cl}$ solution $(10 \mathrm{~mL})$. The phases were separated and the aqueous phase was extracted with EtOAc $(3 \times 10$ $\mathrm{mL}$ ). The combined organic phases were dried over $\mathrm{MgSO}_{4}$, filtered, and concentrated under reduced pressure to afford a crude mixture of coupled product. The residue was used for the next reaction without further purification.

To a round-bottom flask with a magnetic stir bar were added the crude product from the previous step $(1.72 \mathrm{~g}, 6.18 \mathrm{mmol})$ and THF $(21 \mathrm{~mL})$. To this was added TBAF (1.0 M in THF, 5.0 $\mathrm{mL}, 5.0 \mathrm{mmol}$ ), and the resulting solution was stirred for $24 \mathrm{~h}$ at $23{ }^{\circ} \mathrm{C}$. The reaction mixture was quenched by saturated aqueous $\mathrm{NH}_{4} \mathrm{Cl}(20 \mathrm{~mL})$. The phases were separated, and the aqueous phase was extracted with EtOAc $(3 \times 20 \mathrm{~mL})$. The combined organic phases were dried over $\mathrm{MgSO}_{4}$, filtered, and concentrated under reduced pressure. The residue was purified by flash column chromatography (4:1 hexanes:EtOAc) to afford diene allylic alcohol 17 (714 mg, 4.35 mmol, 63\% yield over two steps) as a colorless oil; $\mathrm{R}_{f}=0.67$ (10:1, hexanes:EtOAc) ${ }^{1} \mathrm{H}$ NMR (500 MHz, 
$\left.\mathrm{CDCl}_{3}\right) \delta 6.05-5.95(\mathrm{~m}, 1 \mathrm{H}), 5.83-5.75(\mathrm{~m}, 1 \mathrm{H}), 5.01(\mathrm{dt}, J=7.2,1.9 \mathrm{~Hz}, 1 \mathrm{H}), 2.65-2.53(\mathrm{~m}, 1 \mathrm{H})$, 2.35-2.26 (m, 1H), 2.26-2.10 (m, 3H), 1.87 (ddt, $J=13.9,8.0,2.4 \mathrm{~Hz}, 1 \mathrm{H}), 1.73-1.53(\mathrm{~m}, 5 \mathrm{H})$; ${ }^{13} \mathrm{C}$ NMR $\left(126 \mathrm{MHz}, \mathrm{CDCl}_{3}\right) \delta 146.39,131.82,127.36,125.35,77.16,76.22,33.82,30.48,26.39$, 25.81, 22.81, 22.43; IR (Neat Film, NaCl) 3339, 3045, 2925, 2855, 1435, 1302, 1044, 986, 941, $823 \mathrm{~cm}^{-1}$; HRMS (EI+) $\mathrm{m} / z$ calc'd for $\mathrm{C}_{11} \mathrm{H}_{16} \mathrm{O}[\mathrm{M} \bullet]^{+}:$164.1201, found 164.1170.

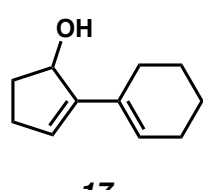

17

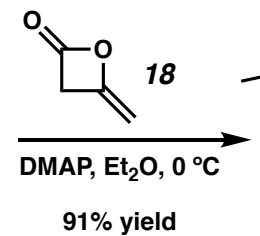

$91 \%$ yield

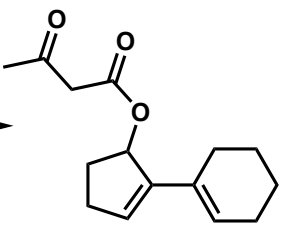

19

2-(Cyclohex-1-en-1-yl)cyclopent-2-en-1-yl 3-oxobutanoate (19): To a flame-dried roundbottom flask equipped with a magnetic stir bar were added allylic alcohol 17 (60 mg, $0.365 \mathrm{mmol})$, 4-dimethylaminopyridine $(0.2 \mathrm{mg}, 0.0016 \mathrm{mmol})$ and $\mathrm{Et}_{2} \mathrm{O}(1.5 \mathrm{~mL})$. The flask was cooled to $0{ }^{\circ} \mathrm{C}$ and stirred for $10 \mathrm{~min}$. Diketene $(\mathbf{1 8}, 0.03 \mathrm{~mL}, 0.389 \mathrm{mmol})$ was added dropwise. The reaction mixture was stirred for $15 \mathrm{~min}$ at $0{ }^{\circ} \mathrm{C}$ then quenched with ice-cold water $(1.5 \mathrm{~mL})$. The mixture was extracted with $\mathrm{Et}_{2} \mathrm{O}(3 \times 3 \mathrm{~mL})$. The combined organic layers were washed by brine $(3 \mathrm{~mL})$, dried over $\mathrm{MgSO}_{4}$, and concentrated under reduced pressure. The crude oil was purified by flash column chromatography (4:1 hexanes, EtOAc) to afford $\beta$-ketoester $19(82.7 \mathrm{mg}, 0.333 \mathrm{mmol}$, $91 \%$ yield) as a colorless oil; $\mathrm{R}_{f}=0.52\left(4: 1\right.$, hexanes:EtOAc); ${ }^{1} \mathrm{H}$ NMR $\left(500 \mathrm{MHz}, \mathrm{CDCl}_{3}\right) \delta 6.04$ $(\mathrm{dt}, J=7.2,1.8 \mathrm{~Hz}, 1 \mathrm{H}), 5.98-5.94(\mathrm{~m}, 1 \mathrm{H}), 5.76-5.72(\mathrm{~m}, 1 \mathrm{H}), 3.43(\mathrm{~s}, 2 \mathrm{H}), 2.61-2.53(\mathrm{~m}, 1 \mathrm{H})$, 2.40-2.24 (m, 2H), $2.22(\mathrm{~s}, 3 \mathrm{H}), 2.21-2.16(\mathrm{~m}, 2 \mathrm{H}), 2.16-2.07(\mathrm{~m}, 2 \mathrm{H}), 1.96-1.88(\mathrm{~m}, 2 \mathrm{H}), 1.71-$ $1.51(\mathrm{~m}, 4 \mathrm{H}) ;{ }^{13} \mathrm{C} \mathrm{NMR}\left(126 \mathrm{MHz}, \mathrm{CDCl}_{3}\right) \delta 200.7,167.3,142.2,131.1,130.7,125.9,79.9,50.7$, 31.6, 30.8, 30.2, 26.6, 25.8, 22.7, 22.3; IR (Neat Film, NaCl) 2926, 2853, 1718, 1643, 1412, 1358, 
1310, 1243, 1147, 1027, 977, 936, 896, $800 \mathrm{~cm}^{-1}$; HRMS (MM) $m / z$ calc'd for $\mathrm{C}_{15} \mathrm{H}_{19} \mathrm{O}_{3}[\mathrm{M}-\mathrm{H}]^{-}$: 247.1340 , found 247.1362 .

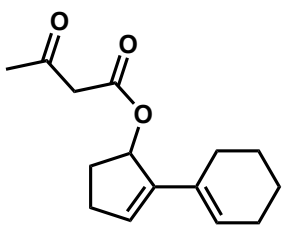

19

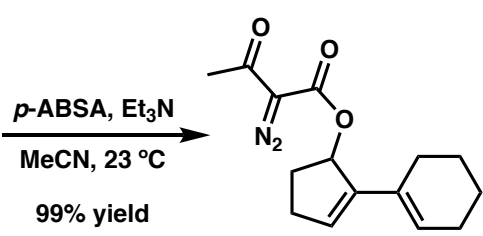

20

2-(Cyclohex-1-en-1-yl)cyclopent-2-en-1-yl 2-diazo-3-oxobutanoate (20): To a roundbottom flask equipped with a magnetic stir bar were added $\beta$-ketoester 19 (80 mg, $0.322 \mathrm{mmol})$, MeCN (3 mL), and p-ABSA (130 mg, $0.541 \mathrm{mmol}) . \mathrm{Et}_{3} \mathrm{~N}(0.2 \mathrm{~mL}, 1.43 \mathrm{mmol})$ was added dropwise. The reaction mixture was stirred for $2 \mathrm{~h}$ at $23{ }^{\circ} \mathrm{C}$. The reaction mixture was filtered through a silica gel plug $\left(2: 1\right.$ pentane: $\left.\mathrm{Et}_{2} \mathrm{O}\right)$ was then concentrated under reduced pressure to afford diazo ester 20 (88.2 mg, $0.322 \mathrm{mmol}$, 99\% yield) as a yellowish oil; $\mathbf{R}_{f}=0.44$ (6:1, hexanes:EtOAc); ${ }^{1} \mathrm{H}$ NMR $\left(500 \mathrm{MHz}, \mathrm{CDCl}_{3}\right) \delta 6.08(\mathrm{dt}, J=1.66 \mathrm{~Hz}, 1.66 \mathrm{~Hz}, 7.75 \mathrm{~Hz}, 1 \mathrm{H}), 5.95$ $(\mathrm{d}, J=2.62 \mathrm{~Hz}, 1 \mathrm{H}), 5.71(\mathrm{~s}, 1 \mathrm{H}), 2.58-2.55(\mathrm{~m}, 1 \mathrm{H}), 2.44(\mathrm{~s}, 3 \mathrm{H}), 2.31-2.24(\mathrm{~m}, 1 \mathrm{H}), 2.22(\mathrm{~s}$, 3H), 2.39-2.26 (m, 2H), 2.18-2.09 (m, 4H), 1.95-1.90 (m, 1H), 1.68-1.52 (m, 4H); ${ }^{13} \mathrm{C}$ NMR (126 $\left.\mathrm{MHz}, \mathrm{CDCl}_{3}\right) \delta 190.5,161.6,142.1,131.2,130.7,125.5,80.3,31.7,30.7,28.4,26.3,25.8,22.7$, 22.3; IR (Neat Film, NaCl) 3298, 3050, 2929, 2856, 2390, 2297, 2208, 2138, 1712, 1661, 1652, $1447,1435,1365,1312,1247,1149,1061,1024,965,926,854,836,816,800,746 \mathrm{~cm}^{-1}$; HRMS $(\mathrm{FAB}+) \mathrm{m} / z$ calc'd for $\mathrm{C}_{15} \mathrm{H}_{19} \mathrm{O}_{3} \mathrm{~N}_{2}[\mathrm{M}+\mathrm{H}]^{-}: 275.1396$, found 275.1389.

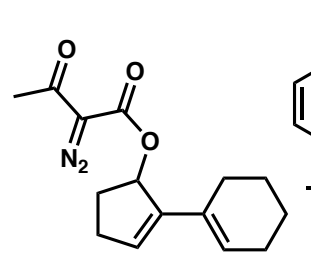

20

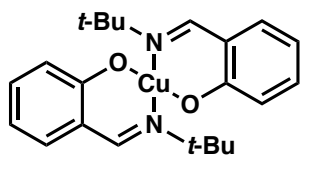

PhMe, $110^{\circ} \mathrm{C}$ $65 \%$ yield

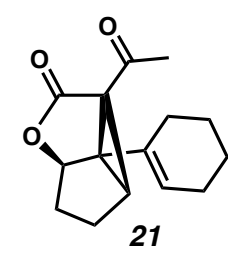

21

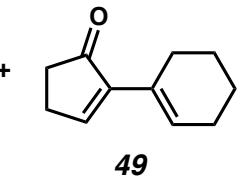

49 


\section{(2aS,2a $\left.{ }^{1} S, 4 a R\right)-2 b-A c e t y l-2 a^{1}-(c y c l o h e x-1-e n-1-y l) h e x a h y d r o-3 H-4-$}

oxacyclopropa $[\boldsymbol{c d}]$ pentalen-3-one (21): To a flame-dried two neck round-bottom flask equipped with a magnetic stir bar was added copper catalyst $(20 \mathrm{mg}, 0.0459 \mathrm{mmol})$ in a nitrogen-filled glove box. The flask was sealed with two rubber septa and removed from the glove box. One of the rubber septa was replaced with a reflux condenser connected to a nitrogen inlet. A solution of diazo ester $20(254.8 \mathrm{mg}, 0.929 \mathrm{mmol})$ in toluene $(46 \mathrm{~mL})$ was added. The reaction was heated to reflux in a $110{ }^{\circ} \mathrm{C}$ oil bath. After $2 \mathrm{~h}$ of stirring, the reaction mixture was cooled to $23{ }^{\circ} \mathrm{C}$ and stirred for $15 \mathrm{~min}$. The mixture was concentrated and purified by flash column chromatography (15:1 hexanes: EtOAc) to afford cyclopropane 21 (148 $\mathrm{mg}, 0.601 \mathrm{mmol}, 65 \%$ yield) as a yellowish oil; $\mathrm{R}_{f}=0.36\left(6: 1\right.$ hexanes:EtOAc); ${ }^{1} \mathrm{H}$ NMR $\left(500 \mathrm{MHz}, \mathrm{CDCl}_{3}\right) \delta 5.72-5.70(\mathrm{~m}, 1 \mathrm{H}), 4.81(\mathrm{~d}, J$ $=1.30 \mathrm{~Hz}, 1 \mathrm{H}), 3.10(\mathrm{~d}, J=6.40 \mathrm{~Hz}, 1 \mathrm{H}), 2.45(\mathrm{~s}, 3 \mathrm{H}), 2.31-2.24(\mathrm{~m}, 1 \mathrm{H}), 2.15-2.12(\mathrm{~m}, 1 \mathrm{H})$, 2.04-1.98 (m, 3H), 1.91-1.85 (m, 1H), 1.80-1.78 (m, 1H), 1.71-1.49 (m, 5H); ${ }^{13} \mathrm{C}$ NMR (126 $\left.\mathrm{MHz}, \mathrm{CDCl}_{3}\right) \delta 197.1,172.9,123.0,128.3,85.3,66.7,51.6,39.4,38.1,30.1,28.3,25.3,24.0,22.6$, 22.0; IR (Neat Film, NaCl) 2929, 1760, 1699, 1435, 1360, 1311, 1243, 1159, 1089, 1008, 979, 956, 925, 906, 855, 799, $756 \mathrm{~cm}^{-1}$; HRMS (MM+) m/z calc'd for $\mathrm{C}_{15} \mathrm{H}_{19} \mathrm{O}_{3}[\mathrm{M}+\mathrm{H}]^{+}:$: 247.1329, found 247.1327 , and dienone $49(22 \mathrm{mg}, 0.136 \mathrm{mmol}, 15 \%$ yield $)$ as a colorless oil; $\mathrm{R}_{f}=0.40(6: 1$ hexanes: EtOAc); ${ }^{1} \mathrm{H}$ NMR $\left(500 \mathrm{MHz} \mathrm{CDCl}_{3}\right) \delta$ 7.39-7.33 (m, 1H), 6.91-6.85 (m, 1H), 2.60$2.54(\mathrm{~m}, 2 \mathrm{H}), 2.51-2.43(\mathrm{~m}, 2 \mathrm{H}), 2.21-2.15(\mathrm{~m}, 4 \mathrm{H}), 1.74-1.67(\mathrm{~m}, 2 \mathrm{H}), 1.65-1.55(\mathrm{~m}, 2 \mathrm{H}) ;{ }^{13} \mathrm{C}$ NMR $\left(126 \mathrm{MHz}, \mathrm{CDCl}_{3}\right) \delta 208.8,155.2,128.8,128.6,36.3,26.8,25.7,25.7,22.8,22.1$; IR (Neat Film, NaCl) 3386, 3051, 2925, 2857, 2834, 2661, 1703, 1699, 1340, 1589, 1439, 1406, 1385, 1342, $1318,1294,1263,1208,1175,1136,1113,1079,1016,998,976,940,926,887,840,832,803$, 785, 762, $724 \mathrm{~cm}^{-1}$; HRMS (FAB+) $m / z$ calc'd for $\mathrm{C}_{11} \mathrm{H}_{15} \mathrm{O}[\mathrm{M}+\mathrm{H}]^{+}:$163.1123, found 163.1128. 


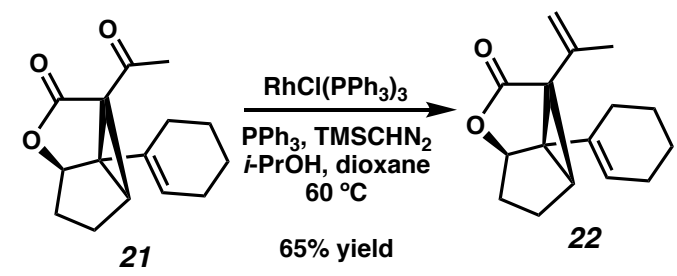

$\left(2 \mathrm{a} S, 2 \mathrm{a}^{1} S, 4 a R\right)-2 \mathrm{a}^{1}-(C y c l o h e x-1-e n-1-y l)-2 b-($ prop-1-en-2-yl)hexahydro-3H-4-

oxacyclopropa $[c d]$ pentalen-3-one (22): To a flame-dried round-bottom flask equipped with a magnetic stir bar were added Wilkinson's catalyst (4.3 mg, $0.00465 \mathrm{mmol})$ and $\mathrm{PPh}_{3}(54 \mathrm{mg}, 0.206$ mmol) in a nitrogen-filled glove box. The flask was sealed with a rubber septum, removed from the glove box and connected to a nitrogen inlet. Dioxane $(2 \mathrm{~mL})$ was added, and the reaction was immersed in a $60^{\circ} \mathrm{C}$ oil bath. $i$-PrOH $(0.21 \mathrm{~mL}, 2.75 \mathrm{mmol})$ was added, followed by a solution of cyclopropane 21 (46 mg, $0.187 \mathrm{mmol})$ in dioxane $(0.5 \mathrm{~mL})$ to give a reddish solution. A solution of trimethylsilyldiazomethane ( $2 \mathrm{M}$ in $\left.\mathrm{Et}_{2} \mathrm{O}, 0.22 \mathrm{~mL}, 0.44 \mathrm{mmol}\right)$ was added to the reaction mixture. The reaction was stirred for $5 \mathrm{~h}$ at $60{ }^{\circ} \mathrm{C}$. The reaction was allowed to cool to ambient temperature and concentrated under reduced pressure. The residue was purified by flash column chromatography (15:1, hexanes: EtOAc) to afford vinyl lactone 22 (30 mg, $0.123 \mathrm{mmol}, 65 \%$ yield) as a colorless oil; $\mathrm{R}_{f}=0.40\left(6: 1\right.$ hexanes: EtOAc); ${ }^{1} \mathrm{H}$ NMR $\left(500 \mathrm{MHz}, \mathrm{C}_{6} \mathrm{D}_{6}\right) \delta 5.30-5.23$ $(\mathrm{m}, 1 \mathrm{H}), 4.96(\mathrm{dd}, J=3.0,1.5 \mathrm{~Hz}, 1 \mathrm{H}), 4.85(\mathrm{dd}, J=1.5,0.8 \mathrm{~Hz}, 1 \mathrm{H}), 4.53(\mathrm{~d}, J=1.0 \mathrm{~Hz}, 1 \mathrm{H})$, $2.06(\mathrm{dd}, J=4.1,3.5 \mathrm{~Hz}, 1 \mathrm{H}), 1.83-1.77(\mathrm{~m}, 5 \mathrm{H}), 1.75-1.60(\mathrm{~m}, 4 \mathrm{H}), 1.58-1.45(\mathrm{~m}, 1 \mathrm{H})$, 1.46-1.25 (m, 5H); ${ }^{13} \mathrm{C}$ NMR $\left(126 \mathrm{MHz}, \mathrm{C}_{6} \mathrm{D}_{6}\right) \delta 173.5,138.4,138.4,125.5,116.5,83.9,58.9$, 50.2, 38.9, 33.3, 28.0, 25.5, 23.6, 23.0, 22.3, 22.0; IR (Neat Film, NaCl), 3498, 2918, 2850, 1960, $1645,1539,1436,1373,1335,1302,1289,1262,1212,1161,1137,1093,1077,1044,1012,997$, 
906, 841, 802, $751 \mathrm{~cm}^{-1}$; HRMS (MM+) m/z calc'd for $\mathrm{C}_{16} \mathrm{H}_{21} \mathrm{O}_{2}[\mathrm{M}+\mathrm{H}]^{+}:$245.1536, found 245.1555 .

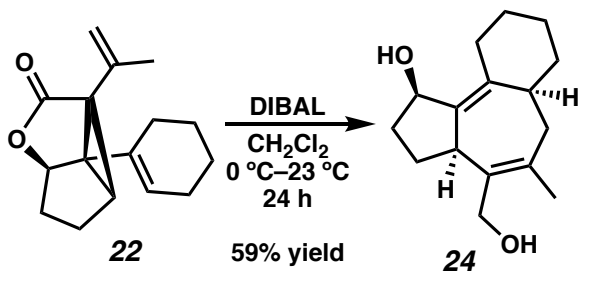

$(1 R, 3 \mathrm{a} R, 6 \mathrm{a} S)-4-(H y d r o x y m e t h y l)-5-m e t h y l-1,2,3,3 \mathrm{a}, 6,6 \mathrm{a}, 7,8,9,10-$

decahydrobenzo[ $e$ ]azulen-1-ol (24): To a flame-dried round-bottom flask equipped with a magnetic stir bar were added vinyl lactone $22(10 \mathrm{mg}, 0.0403 \mathrm{mmol})$ and DCM (1 mL). The flask was cooled to $0{ }^{\circ} \mathrm{C}$ and stirred for $10 \mathrm{~min}$. A solution of DIBAL (1 M in DCM, 0.4 mL, 0.4 mmol) was added dropwise. The reaction mixture was slowly warmed up to $23{ }^{\circ} \mathrm{C}$ and stirred for an additional $24 \mathrm{~h}$. The reaction was quenched with methanol $(0.4 \mathrm{~mL})$. Saturated aqueous potassium sodium tartrate solution $(1 \mathrm{~mL})$ was added to the mixture. The phases were separated, and the aqueous phases were extracted with DCM $(5 \times 2 \mathrm{~mL})$. The combined organic phases were dried over $\mathrm{MgSO}_{4}$, filtered, and concentrated under reduced pressure. The residue was purified by flash column chromatography (2:1, hexanes: EtOAc) to afford diol $\mathbf{2 4}$ as a white solid (6 $\mathbf{m g}, 0.024$ mmol, 59\% yield); $\mathrm{R}_{f}=0.08(2: 1$ hexanes:EtOAc $) ;{ }^{1} \mathrm{H}$ NMR $\left(500 \mathrm{MHz}, \mathrm{C}_{6} \mathrm{D}_{6}\right) \delta 4.61(\mathrm{~d}, J=4.2$ $\mathrm{Hz}, 1 \mathrm{H}), 4.20(\mathrm{~d}, J=11.3 \mathrm{~Hz}, 1 \mathrm{H}), 3.96(\mathrm{~d}, J=11.3 \mathrm{~Hz}, 1 \mathrm{H}), 3.58-3.49(\mathrm{~m}, 1 \mathrm{H}), 3.04(\mathrm{dd}, J=$ 13.6, 4.1 Hz, 1H), $2.75(\mathrm{dd}, J=12.8,3.5 \mathrm{~Hz}, 1 \mathrm{H}), 2.41(\mathrm{qd}, J=12.4,6.1 \mathrm{~Hz}, 1 \mathrm{H}), 1.95-1.83(\mathrm{~m}$, 2H), 1.76-1.67 (m, 5H), 1.64-1.57 (m, 1H), $1.52(\mathrm{dd}, J=13.6,3.6 \mathrm{~Hz}, 1 \mathrm{H}), 1.43-1.27(\mathrm{~m}, 6 \mathrm{H})$; ${ }^{13} \mathrm{C}$ NMR $\left(126 \mathrm{MHz}, \mathrm{C}_{6} \mathrm{D}_{6}\right) \delta 138.9,138.7,138.3,134.2,73.2,60.1,41.6,40.5,38.5,34.8,34.6$, 34.2, 30.2, 29.4, 27.6, 26.5, 21.9; IR (Neat Film, NaCl) 3338, 2927, 2853, 1740, 1447, 1373, 1242, 
1177, 1043, 965, $913 \mathrm{~cm}^{-1}$; HRMS (FAB+) m/z calc'd for $\mathrm{C}_{16} \mathrm{H}_{23} \mathrm{O}_{2}\left[\mathrm{M}+\mathrm{H}-\mathrm{H}_{2}\right]^{+}:$247.1698, found 247.1692.

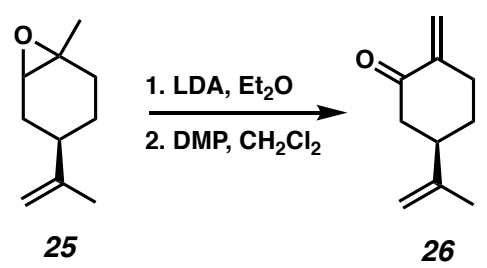

(R)-2-Methylene-5-(prop-1-en-2-yl)cyclohexan-1-one (26): To a flame-dried round-bottom flask with a magnetic stir bar were added diisopropyl amine $(1.75 \mathrm{~mL}, 13.3 \mathrm{mmol})$ and $\mathrm{Et}_{2} \mathrm{O}(35$ $\mathrm{mL})$. A solution of $n$-BuLi (2.12 M in hexane, $6.84 \mathrm{~mL}, 14.5 \mathrm{mmol})$ was added dropwise over a period of $30 \mathrm{~min}$. A solution of epoxide $25(2 \mathrm{~mL}, 12.1 \mathrm{mmol})$ in $\mathrm{Et}_{2} \mathrm{O}(7 \mathrm{~mL})$ was added dropwise over a period of $30 \mathrm{~min}$. The resulting mixture was allowed to warm up to $23{ }^{\circ} \mathrm{C}$ and then stirred for $7 \mathrm{~h}$. The reaction mixture was cooled in ice bath and water was added. The organic phase was separated and washed with $2 \mathrm{M}$ aqueous $\mathrm{HCl}(10 \mathrm{~mL})$, water $(10 \mathrm{~mL})$, saturated aqueous $\mathrm{NaHCO}_{3}$ $(10 \mathrm{~mL})$ and brine $(10 \mathrm{~mL})$. The $\mathrm{Et}_{2} \mathrm{O}$ extracts are combined, dried over $\mathrm{MgSO}_{4}$, and evaporated to afford crude mixture. The residue was used for the next reaction without further purification. To a round-bottom flask equipped with a magnetic stir bar were added semi-crude allylic alcohol (124 mg, $0.815 \mathrm{mmol})$ and DCM (10 mL). Dess-Martin periodinane (440 mg, $1.06 \mathrm{mmol})$ was added to the mixture. The reaction was stirred for $3 \mathrm{~h}$ at $23{ }^{\circ} \mathrm{C}$. The reaction mixture was diluted with $\mathrm{Et}_{2} \mathrm{O}(10 \mathrm{~mL})$ and then a 1:1:1 mixture of saturated aqueous $\mathrm{Na}_{2} \mathrm{~S}_{2} \mathrm{O}_{3}(10 \mathrm{~mL})$, saturated aqueous $\mathrm{NaHCO}_{3}(10 \mathrm{~mL})$, and water $(10 \mathrm{~mL})$ was added slowly. The resulting mixture was stirred for 20 min resulting in two clear layers. The organic layer was gathered, and the aqueous layer was extracted with $\mathrm{Et}_{2} \mathrm{O}(30 \mathrm{~mL} \times 3)$. The organic layers were combined and dried over $\mathrm{Na}_{2} \mathrm{SO}_{4}$, and evaporated to afford crude mixture (Caution, the solvent was only partially removed, as enone $\mathbf{2 6}$ dimerizes easily.) The mixture was filtered through silica gel (8:1 pentane: $\left.\mathrm{Et}_{2} \mathrm{O}\right)$ and used in the 
next reaction without further purification. The characterization data matched those reported in the literature. 5

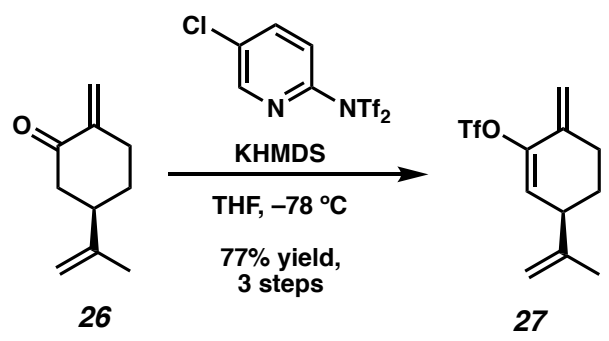

(R)-6-Methylene-3-(prop-1-en-2-yl)cyclohex-1-en-1-yl trifluoromethanesulfonate (27):

To a flame-dried round-bottom flask equipped with a magnetic stir bar was added potassium bis(trimethylsilyl)amide $(310 \mathrm{mg}, 1.55 \mathrm{mmol})$ in a nitrogen filled glove box. The flask was sealed with rubber septum and removed from the glove box, connected to a nitrogen inlet, and cooled to $-78^{\circ} \mathrm{C}$. A solution of semi-crude enone $\mathbf{2 6}(150 \mathrm{mg}, 1 \mathrm{mmol})$ in THF (10 mL) was added dropwise by syringe pump over $2 \mathrm{~h}$. After addition of enone 26 was completed, Comins reagent (652 mg, $1.66 \mathrm{mmol})$ in THF $(10 \mathrm{~mL})$ was added dropwise. After stirring for $4 \mathrm{~h}$ at $-78{ }^{\circ} \mathrm{C}$, the reaction mixture was poured into saturated aqueous $\mathrm{NaHCO}_{3}(50 \mathrm{~mL})$ and allowed to warm to $23{ }^{\circ} \mathrm{C}$. The mixture was extracted with $\mathrm{Et}_{2} \mathrm{O}(30 \times 3 \mathrm{~mL})$. The combined organic layers were washed with brine $(100 \mathrm{~mL})$, dried over $\mathrm{MgSO}_{4}$, and concentrated under reduced pressure. The residue was purified by flash column chromatography (25:1 hexanes: EtOAc) to afford triflate 27 (218 $\mathrm{mg}$, $0.77 \mathrm{mmol}, 77 \%$ yield over 3 steps $) ; \mathrm{R}_{f}=0.52$ (4:1, hexanes: EtOAc); ${ }^{1} \mathrm{H}$ NMR $\left(500 \mathrm{MHz}, \mathrm{CDCl}_{3}\right)$ $\delta 5.82(\mathrm{dd}, J=4.0,1.7 \mathrm{~Hz}, 1 \mathrm{H}), 5.28(\mathrm{~s}, 1 \mathrm{H}), 5.06-4.99(\mathrm{~m}, 1 \mathrm{H}), 4.88(\mathrm{t}, J=1.5 \mathrm{~Hz}, 1 \mathrm{H}), 4.77(\mathrm{dt}$, $J=1.7,0.9 \mathrm{~Hz}, 1 \mathrm{H}), 3.14-3.06(\mathrm{~m}, 1 \mathrm{H}), 2.63-2.49(\mathrm{~m}, 1 \mathrm{H}), 2.48-2.37(\mathrm{~m}, 1 \mathrm{H}), 1.95-1.83(\mathrm{~m}$, 1H), 1.77 (s, 3H), 1.72-1.60 (m, 1H); $\left.{ }^{13} \mathrm{C} \mathrm{NMR} \mathrm{(126} \mathrm{MHz,} \mathrm{CDCl}_{3}\right) \delta 149.5,147.1,145.8,144.0$, $139.5,136.5,136.0,126.3,123.9,120.7,119.9,117.4,112.8,112.0,111.1,1102,43.4,29.6,27.0$, 
21.3; IR (Neat Film, NaCl) 3084, 2947, 2869, 1648, 1608, 1447, 1436, 1422, 1428, 1373, 1245 , 1214, 1143, 1129, 1066, 1045, 1017, 998, 978, 948, 755, $737 \mathrm{~cm}^{-1}$; HRMS (FAB+) m/z calc'd for $\mathrm{C}_{11} \mathrm{H}_{12} \mathrm{~F}_{3} \mathrm{O}_{3} \mathrm{~S}[\mathrm{M}+\mathrm{H}-\mathrm{H}]^{+}:$281.0459, found $281.0473 ;[\alpha]_{\mathrm{D}}^{25.0} 61.1^{\circ}\left(c 0.25, \mathrm{CHCl}_{3}\right)$.

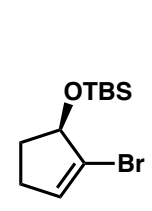

$(+)-14$

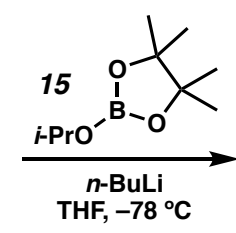

$89 \%$ yield

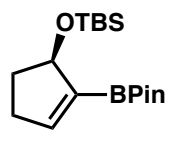

$(+)-12$

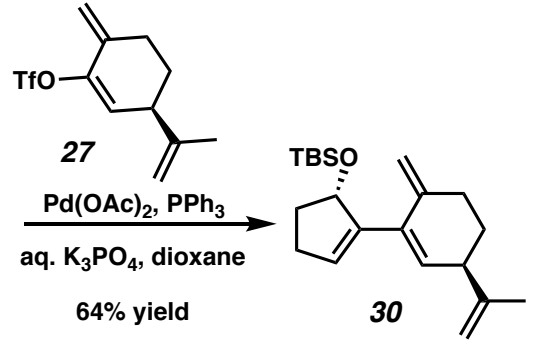

tert-Butyldimethyl $(((S)-2-((R)-6-m e t h y l e n e-3-(p r o p-1-e n-2-y l) c y c l o h e x-1-e n-1-$

yl)cyclopent-2-en-1-yl)oxy)silane (30): To a flame-dried round-bottom flask with a magnetic stir bar were added bromide (-)-14 (6.0 g, $21.6 \mathrm{mmol})$ and THF (70 mL). The flask was cooled to -78 ${ }^{\circ} \mathrm{C}$ and stirred for $10 \mathrm{~min}$, after which $n$-BuLi (2.5 M in hexanes, $\left.13 \mathrm{~mL}, 32.5 \mathrm{mmol}\right)$ was added dropwise. The reaction mixture was stirred at $-78{ }^{\circ} \mathrm{C}$ for $30 \mathrm{~min}$ and isopropyl pinacolyl borate $(\mathbf{1 5}, 6.9 \mathrm{~mL}, 33.8 \mathrm{mmol})$ was added. The reaction mixture was stirred at $-78{ }^{\circ} \mathrm{C}$ for $30 \mathrm{~min}$ and quenched with $\mathrm{HCl}$ solution ( $2 \mathrm{~N}_{\text {in }} \mathrm{Et}_{2} \mathrm{O}, 16.3 \mathrm{~mL}, 32.5 \mathrm{mmol}$ ). Following addition, the reaction mixture was diluted with $\mathrm{Et}_{2} \mathrm{O}(70 \mathrm{~mL})$ and warmed up to $23^{\circ} \mathrm{C}$. The reaction mixture was filtered and was concentrated under reduced pressure, and the residue was used in the next reaction without further purification.

To a flame-dried round-bottom flask equipped with a magnetic stir bar were added semi-crude boronate (-)-12 (2.65 g, $7.74 \mathrm{mmol})$, triflate $27(1.987 \mathrm{~g}, 7.04 \mathrm{mmol})$, palladium acetate $(82 \mathrm{mg}$, $0.35 \mathrm{mmol})$, triphenylphosphine $(199 \mathrm{mg}, 0.70 \mathrm{mmol})$, tribasic potassium phosphate $(4.5 \mathrm{~g}, 21$ mmol). The mixture was evacuated and back filled with argon (x3). The mixture was dissolved in dioxane $(25 \mathrm{~mL})$ then added water $(2.5 \mathrm{~mL})$. The reaction mixture was stirred at $23{ }^{\circ} \mathrm{C}$ for $40 \mathrm{~h}$. The resulting mixture was then diluted with EtOAc $(25 \mathrm{~mL})$, washed by saturated aqueous $\mathrm{NH}_{4} \mathrm{Cl}$ 
(25 mL), and then dried over $\mathrm{MgSO}_{4}$. The mixture was filtered and concentrated under reduced pressure to afford crude mixture of $\mathbf{3 0}$ as a colorless oil. The residue was purified by flash column chromatography (25:1 hexanes:EtOAc) to afford diene 30 (1.5 g, 4.54 mmol, 64\% yield over triflate 27) $\mathrm{R}_{f}=0.95\left(10: 1\right.$, hexanes:EtOAc); ${ }^{1} \mathrm{H}$ NMR $\left(400 \mathrm{MHz}, \mathrm{C}_{6} \mathrm{D}_{6}\right) \delta 5.88-5.84(\mathrm{~m}, 1 \mathrm{H})$, 5.70-5.68 (m, 1H), 5.02-4.93 (m, 2H), 4.93-4.88 (m, 2H), 4.85-4.81 (m, 1H), 2.97-2.91 (m, 1H), 2.51-2.30 (m, 4H), 2.16-2.02 (m, 2H), $1.80(\mathrm{tt}, J=8.3,4.0 \mathrm{~Hz}, 2 \mathrm{H}), 1.72-1.56(\mathrm{~m}, 2 \mathrm{H}), 1.00(\mathrm{~s}$, 9H), $0.09(\mathrm{~s}, 6 \mathrm{H}) ;{ }^{13} \mathrm{C} \mathrm{NMR}\left(101 \mathrm{MHz}, \mathrm{C}_{6} \mathrm{D}_{6}\right) \delta 148.5,146.7,143.4,135.9,132.7,130.9,111.0$, 110.7, 78.7, 45.1, 34.8, 32.1, 29.3, 26.2, 26.0, 20.9, 18.4, -4.3, -4.5; IR (Neat Film, NaCl) 3435, $3080,2956,2929,2856,2360,1725,1645,1472,1463,1362,1258,1095,1020,947,865,836$, 801, $776 \mathrm{~cm}^{-1}$; HRMS (FAB+) m/z calc'd for $\mathrm{C}_{21} \mathrm{H}_{33} \mathrm{OSi}\left[\mathrm{M}+\mathrm{H}-\mathrm{H}_{2}\right]^{+}:$329.2301, found 329.2297; $[\alpha]_{\mathrm{D}}^{25.0}-38.3^{\circ}\left(c 0.150, \mathrm{CHCl}_{3}\right)$.

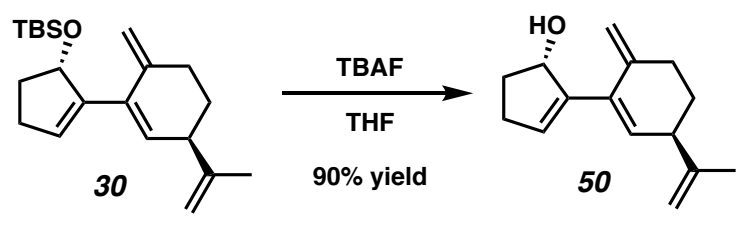

(S)-2-((R)-6-Methylene-3-(prop-1-en-2-yl)cyclohex-1-en-1-yl)cyclopent-2-en-1-ol (50): To a round-bottom flask with a magnetic stir bar were added silyl ether $\mathbf{3 0}$ (1.5 g, $4.54 \mathrm{mmol})$ and THF (23 mL). To the mixture was added TBAF (1.0 M in THF, $7.7 \mathrm{~mL}, 7.7 \mathrm{mmol})$ and stirred for $24 \mathrm{~h}$ at $23{ }^{\circ} \mathrm{C}$. The reaction mixture was quenched with sat. aq. $\mathrm{NH}_{4} \mathrm{Cl}(20 \mathrm{~mL})$ and extracted with $\mathrm{Et}_{2} \mathrm{O}(3 \times 10 \mathrm{~mL})$. The organic layers were combined, dried over $\mathrm{MgSO}_{4}$, and concentrated under reduced pressure. The residue was purified by flash column chromatography (3:1 hexanes: EtOAc) to afford allylic alcohol $\mathbf{5 0}(1.23 \mathrm{~g}, 5.69 \mathrm{mmol}, 90 \%$ yield $)$ as a colorless oil; $\mathrm{R}_{f}=0.10(10: 1$, hexanes: EtOAc); ${ }^{1} \mathrm{H}$ NMR (400 MHz, $\left.\mathrm{C}_{6} \mathrm{D}_{6}\right)$ $85.84-5.79(\mathrm{~m}, 1 \mathrm{H}), 5.76-5.71(\mathrm{~m}, 1 \mathrm{H}), 5.11-5.05$ $(\mathrm{m}, 1 \mathrm{H}), 4.95-4.86(\mathrm{~m}, 3 \mathrm{H}), 4.85-4.80(\mathrm{~m}, 1 \mathrm{H}), 2.92-2.81(\mathrm{~m}, 1 \mathrm{H}), 2.43-2.21(\mathrm{~m}, 3 \mathrm{H}), 2.19-1.98$ 
$(\mathrm{m}, 2 \mathrm{H}), 1.85-1.68(\mathrm{~m}, 2 \mathrm{H}), 1.66-1.45(\mathrm{~m}, 4 \mathrm{H}), 1.21(\mathrm{~d}, J=5.8 \mathrm{~Hz}, 1 \mathrm{H}) ;{ }^{13} \mathrm{C} \mathrm{NMR}(101 \mathrm{MHz}$, $\left.\mathrm{C}_{6} \mathrm{D}_{6}\right) \delta 148.6,146.0,143.4,135.1,132.2,131.2,128.4,128.3,128.2,128.1,127.9,127.8,111.2$, 111.1, 78.0, 45.0, 33.9, 32. 5, 30.3, 29.5, 20.7; IR (Neat Film, NaCl) 3774, 3659, 3078, 3042, 2935, 2852, 2112, 1644, 1442, 1373, 1311, 1166, 1047, 930, 889, $843 \mathrm{~cm}^{-1}$; HRMS (FAB+) m/z calc'd for $\mathrm{C}_{15} \mathrm{H}_{19} \mathrm{O}_{3}\left[\mathrm{M}+\mathrm{H}-\mathrm{H}_{2}\right]^{+}:$215.1436, found 215.1441; $[\alpha]_{\mathrm{D}}^{25.0}-16.2^{\circ}\left(c 0.150, \mathrm{CHCl}_{3}\right)$.

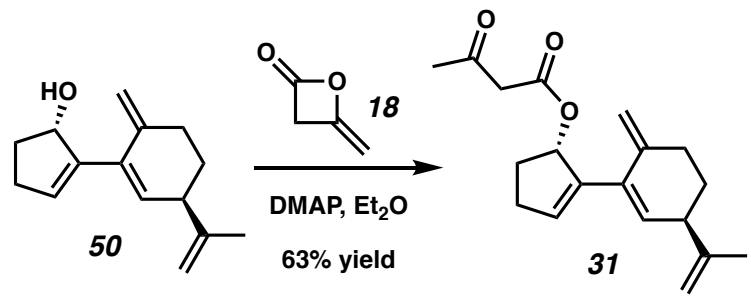

(S)-2-((R)-6-Methylene-3-(prop-1-en-2-yl)cyclohex-1-en-1-yl)cyclopent-2-en-1-yl

oxobutanoate (31): To a flame-dried round-bottom flask with a magnetic stir bar were added allylic alcohol $\mathbf{5 0}$ (1.23 g, $5.69 \mathrm{mmol})$, 4-dimethylaminopyridine (35 mg, $0.29 \mathrm{mmol})$ and $\mathrm{Et}_{2} \mathrm{O}$ (20 mL). The flask was cooled to $0{ }^{\circ} \mathrm{C}$ and stirred for $10 \mathrm{~min}$. Diketene $(\mathbf{1 8}, 0.5 \mathrm{~mL}, 6.48 \mathrm{mmol})$ was added dropwise. The reaction mixture was stirred $15 \mathrm{~min}$ at $0{ }^{\circ} \mathrm{C}$ was then quenched by icecold water $(10 \mathrm{~mL})$. The mixture was extracted with $\mathrm{Et}_{2} \mathrm{O}(3 \times 15 \mathrm{~mL})$. The combined organic layers were washed by brine $(15 \mathrm{~mL})$, dried over $\mathrm{MgSO}_{4}$, and concentrated under reduced pressure. The crude oil was purified by flash column chromatography (10:1 hexanes: EtOAc) to afford $\beta$ ketoester 31 (1.07 g, $3.56 \mathrm{mmol}, 63 \%$ yield) as a colorless oil; $\mathrm{R}_{f}=0.40$ (3:1, hexanes:Et $\left.{ }_{2} \mathrm{O}\right) ;{ }^{1} \mathrm{H}$ NMR $\left(400 \mathrm{MHz}, \mathrm{C}_{6} \mathrm{D}_{6}\right) \delta 6.23-6.15(\mathrm{~m}, 1 \mathrm{H}), 5.82-5.80(\mathrm{~m}, 1 \mathrm{H}), 5.80-5.77(\mathrm{~m}, 1 \mathrm{H}), 5.05(\mathrm{~d}, J=$ $2.1 \mathrm{~Hz}, 1 \mathrm{H}), 4.97-4.81(\mathrm{~m}, 3 \mathrm{H}), 2.94(\mathrm{~s}, 2 \mathrm{H}), 2.92-2.83(\mathrm{~m}, 1 \mathrm{H}), 2.43-2.23(\mathrm{~m}, 3 \mathrm{H}), 2.23-2.11$ (m, 1H), 2.08-1.92 (m, 1H), 1.92-1.83 (m, 1H), 1.82-1.73 (m, 1H), $1.68(\mathrm{~s}, 3 \mathrm{H}), 1.65(\mathrm{~s}, 3 \mathrm{H})$, $1.62-1.50(\mathrm{~m}, 1 \mathrm{H}) ;{ }^{13} \mathrm{C} \mathrm{NMR}\left(101 \mathrm{MHz}, \mathrm{C}_{6} \mathrm{D}_{6}\right) \delta 199.0,169.0,166.9,148.5,143.2,141.6,134.9$, 132.1, 111.2, 111.1, 81.3, 50.1, 45.0, 32.4, 31.1, 30.8, 29.54, 29.47, 20.8; IR (Neat Film, NaCl) 
$3629,3078,2935,2855,1727,1644,1440,1360,1315,1238,1149,1029,934,895,847,802,739$

$\mathrm{cm}^{-1}$; HRMS (FAB+) m/z calc'd for $\mathrm{C}_{19} \mathrm{H}_{25} \mathrm{O}_{3}[\mathrm{M}+\mathrm{H}]^{+*}: 301.1804$, found 301.1814; $[\alpha]_{\mathrm{D}}{ }^{25.0}-41.8^{\circ}$ $\left(c 0.150, \mathrm{CHCl}_{3}\right)$.
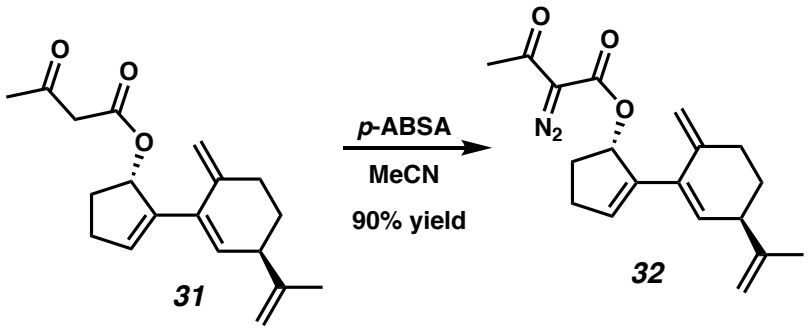

(S)-2-((R)-6-Methylene-3-(prop-1-en-2-yl)cyclohex-1-en-1-yl)cyclopent-2-en-1-yl

2-

diazo-3-oxobutanoate (32): To a round-bottom flask equipped with a magnetic stir bar were added $\beta$-ketoester 31 (1.07 g, $3.56 \mathrm{mmol})$, $\mathrm{MeCN}$ (36 mL), and $p$-ABSA (1.3 g, $5.41 \mathrm{mmol})$. $\mathrm{Et}_{3} \mathrm{~N}$ (1.5 mL, $10.75 \mathrm{mmol})$ was added dropwise. The reaction mixture was stirred for $2 \mathrm{~h}$ at $23^{\circ} \mathrm{C}$. The reaction mixture was filtered through a silica gel plug (pentanes: $\operatorname{Et}_{2} \mathrm{O} 2: 1$ ) and concentrated under reduced pressure to afford diazo ester $32(1.04 \mathrm{~g}, 3.19 \mathrm{mmol}, 90 \%$ yield $)$ as a yellowish oil; $\mathrm{R}_{f}=$ 0.44 (4:1, hexanes: EtOAc); ${ }^{1} \mathrm{H}$ NMR $\left(500 \mathrm{MHz}, \mathrm{CDCl}_{3}\right) \delta$ 6.06-5.98 (m, 2H), 5.61 (dd, $J=2.9$, $1.5 \mathrm{~Hz}, 1 \mathrm{H}), 4.91-4.87(\mathrm{~m}, 2 \mathrm{H}), 4.76(\mathrm{dd}, J=2.0,1.4 \mathrm{~Hz}, 1 \mathrm{H}), 4.74-4.69(\mathrm{~m}, 1 \mathrm{H}), 2.93(\mathrm{ddd}, J=$ 9.1, 5.4, 3.2 Hz, 1H), 2.65-2.54 (m, 1H), 2.51-2.40 (m, 6H), 2.36-2.27 (m, 1H), 2.00-1.88 (m, 2H), $1.71(\mathrm{dd}, J=1.4,0.8 \mathrm{~Hz}, 3 \mathrm{H}), 1.60-1.52(\mathrm{~m}, 1 \mathrm{H}) ;{ }^{13} \mathrm{C} \mathrm{NMR}\left(126 \mathrm{MHz}, \mathrm{CDCl}_{3}\right) \delta 190.5$, $161.4,148.3,142.9,140.8,135.2,134.1,132.1,132.1,110.9,110.9,82.2,44.6,31.9,31.0,30.7$, 29.1, 28.4, 20.8; IR (Neat Film, NaCl) 3794, 3417, 3301, 3078, 2932, 2855, 2617, 2486, 2391, $2301,2210,2135,1953,1713,1659,1441,1361,1307,1247,1151,1063,1025,965,895,847$ $\mathrm{cm}^{-1}$; HRMS (FAB+) $m / z$ calc'd for $\mathrm{C}_{19} \mathrm{H}_{23} \mathrm{O}_{3} \mathrm{~N}_{2}[\mathrm{M}+\mathrm{H}]^{+}: 327.1709$, found $327.1725 ;[\alpha]_{\mathrm{D}}^{25.0}-6.7^{\circ}$ (c $\left.0.250, \mathrm{CHCl}_{3}\right)$. 


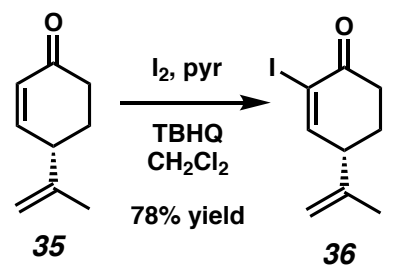

(S)-2-Iodo-4-(prop-1-en-2-yl)cyclohex-2-en-1-one (36): To a round-bottom flask equipped with a magnetic stir bar were added ketone $\mathbf{3 5}^{6}$ (200 mg, $\left.1.47 \mathrm{mmol}\right)$, DCM (35 mL), and tertbutylhydroquinone $(5 \mathrm{mg}, 0.03 \mathrm{mmol})$. A solution of iodine $(700 \mathrm{mg}, 2.76 \mathrm{mmol})$ in pyridine $(1.5$ $\mathrm{mL}, 10.75 \mathrm{mmol}$ ) was added. The reaction mixture was stirred for $2 \mathrm{~h}$ at $23{ }^{\circ} \mathrm{C}$. The reaction was diluted with $\mathrm{Et}_{2} \mathrm{O}(20 \mathrm{~mL})$ and water $(20 \mathrm{~mL})$ and quenched by saturated aqueous $\mathrm{Na}_{2} \mathrm{~S}_{2} \mathrm{O}_{3}(20$ $\mathrm{mL})$. The phases were separated and the aqueous phases were extracted with DCM (3 x $20 \mathrm{~mL})$. The combined organic phases were dried over $\mathrm{MgSO}_{4}$, filtered and concentrated under reduced pressure. The crude product was purified by flash column chromatography (15:1, hexanes: EtOAc) to afford iodide 36 (300 mg, $1.14 \mathrm{mmol}, 78 \%$ yield) as a yellowish oil; $\mathrm{R}_{f}=0.40$ (6:1, hexanes:EtOAc); ${ }^{1} \mathrm{H}$ NMR $\left.\left(400 \mathrm{MHz}, \mathrm{C}_{6} \mathrm{D}_{6}\right)\right) \delta 7.17(\mathrm{~d}, J=1.1 \mathrm{~Hz}, 1 \mathrm{H}), 4.62-4.55(\mathrm{~m}, 1 \mathrm{H}), 4.47-$ $4.43(\mathrm{~m}, 1 \mathrm{H}), 2.36-2.22(\mathrm{~m}, 2 \mathrm{H}), 1.92(\mathrm{ddd}, J=16.2,11.2,4.8 \mathrm{~Hz}, 1 \mathrm{H}), 1.40-1.31(\mathrm{~m}, 1 \mathrm{H}), 1.31-$ $1.20(\mathrm{~m}, 4 \mathrm{H}) ;{ }^{13} \mathrm{C}$ NMR $\left(101 \mathrm{MHz}, \mathrm{C}_{6} \mathrm{D}_{6}\right) \delta 190.5,160.2,144.5,128.4,128.3,128.1,127.9,127.8$, 112.8, 105.1, 47.5, 35.4, 27.7, 20.9; IR (Neat Film, NaCl) 3357, 3077, 2951, 2867, 1683, 1645, $1585,1450,1414,1376,1325,1278,1217,1170,1151,1128,1081,1036,971,952,89,805,713$, $644 \mathrm{~cm}^{-1}$; HRMS $(\mathrm{FAB}+) \mathrm{m} / z$ calc'd for $\mathrm{C}_{9} \mathrm{H}_{12} \mathrm{OI}[\mathrm{M}+\mathrm{H}]^{+}: 262.9933$, found $262.9936 ;[\alpha]_{\mathrm{D}}^{25.0}{ }_{-}$ $40.1^{\circ}\left(c 0.44, \mathrm{CHCl}_{3}\right)$.

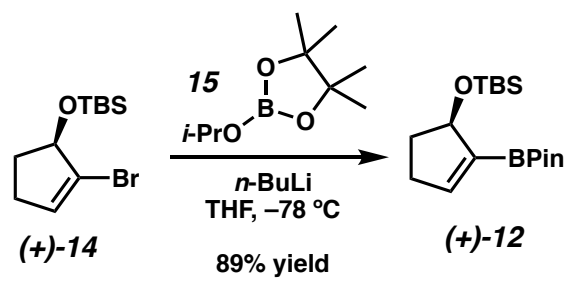


(R)-tert-butyldimethyl((2-(4,4,5,5-tetramethyl-1,3,2-dioxaborolan-2-yl)cyclopent-2-en-1-

yl)oxy)silane ((+)-12): To a round-bottom flask equipped with a magnetic stir bar were added bromide (+)-14 (1.04 g, $3.82 \mathrm{mmol})$ and THF (15 mL). The flask was cooled to $-78{ }^{\circ} \mathrm{C}$ and stirred for $10 \mathrm{~min} . n$-BuLi solution (2.5 $\mathrm{M}$ in hexanes, $2.3 \mathrm{~mL}, 5.75 \mathrm{mmol}$ ) was added dropwise. The reaction mixture was stirred at $-78^{\circ} \mathrm{C}$ for $30 \mathrm{~min}$ and isopropyl pinacolyl borate $(1.2 \mathrm{~mL}, 5.88$ mmol) was added. The reaction mixture was stirred at $-78{ }^{\circ} \mathrm{C}$ for 30 min then quenched with $\mathrm{HCl}$ solution ( $2 \mathrm{~N}$ in $\mathrm{Et}_{2} \mathrm{O}, 2.9 \mathrm{~mL}, 5.8 \mathrm{mmol}$ ). Following addition, the reaction mixture was diluted with diethyl ether $(15 \mathrm{~mL})$ and warmed up to $23{ }^{\circ} \mathrm{C}$. The reaction mixture was filtered and was concentrated under reduced pressure to afford boronate (+)-12 (1.1 g, 3.39 mmol, 89\% yield) as a colorless oil. The characterization data matched those of rac-12. $[\alpha]_{\mathrm{D}}^{25.0} 9.8^{\circ}\left(c 1.35, \mathrm{CHCl}_{3}\right)$.

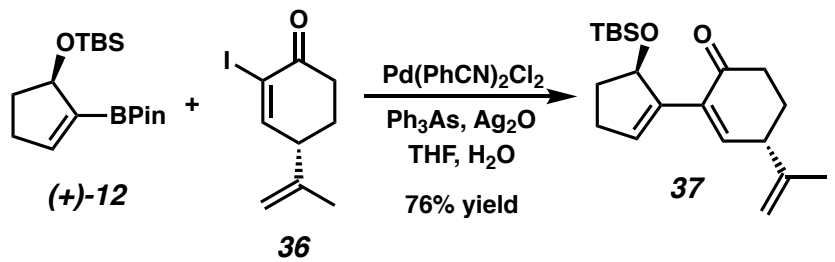

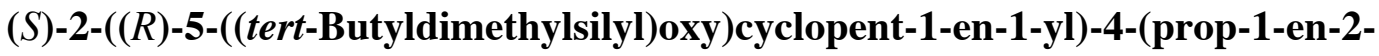

yl)cyclohex-2-en-1-one (37): To a flame-dried round-bottom flask equipped with a magnetic stir bar were added boronate (+)-12 (92 mg, $0.28 \mathrm{mmol})$, iodide 36 (50 mg, $0.19 \mathrm{mmol})$, silver oxide (70 mg, $0.30 \mathrm{mmol})$, triphenylarsine $(6 \mathrm{mg}, 0.02 \mathrm{mmol})$. The mixture was evacuated and backfilled with argon $(\mathrm{x} 3)$. The mixture was dissolved in dioxane $(25 \mathrm{~mL})$ and water $(2.5 \mathrm{~mL})$. To the mixture was added bis(benzonitrile)palladium chloride $(4 \mathrm{mg}, 0.01 \mathrm{mmol})$. The reaction was stirred at $23{ }^{\circ} \mathrm{C}$ for $6 \mathrm{~h}$. The resulting mixture was filtered through celite with EtOAc and the solvent was removed under reduced pressure. The crude product was purified by flash column chromatography (20:1, hexanes: EtOAc) to afford bicycle 37 (48 mg, $0.144 \mathrm{mmol}, 76 \%$ yield over 
36) as a white solid; $\mathrm{R}_{f}=0.54$ (6:1, hexanes: EtOAc); ${ }^{1} \mathrm{H}$ NMR $\left(400 \mathrm{MHz}, \mathrm{C}_{6} \mathrm{D}_{6}\right) \delta 6.72(\mathrm{dd}, J=$ 3.4, 1.3 Hz, 1H), 6.26-6.17 (m, 1H), 5.33-5.25 (m, 1H), 4.76-4.74 (m, 1H), 4.72-4.70 (m, 1H), $2.72(\mathrm{dt}, J=8.5,4.1 \mathrm{~Hz}, 1 \mathrm{H}), 2.51-2.29(\mathrm{~m}, 2 \mathrm{H}), 2.26-1.99(\mathrm{~m}, 3 \mathrm{H}), 1.79-1.62(\mathrm{~m}, 2 \mathrm{H}), 1.62-$ $1.45(\mathrm{~m}, 4 \mathrm{H}), 0.96(\mathrm{~s}, 9 \mathrm{H}), 0.11(\mathrm{~s}, 3 \mathrm{H}), 0.09(\mathrm{~s}, 3 \mathrm{H}) ;{ }^{13} \mathrm{C} \mathrm{NMR}\left(101 \mathrm{MHz}, \mathrm{C}_{6} \mathrm{D}_{6}\right) \delta$ 197.0, 147.9, $146.2,143.0,135.7,132.4,128.3,128.2,128.1,127.9,127.8,112.3,78.5,44.4,38.1,34.6,30.6$, 27.9, 26.2, 21.2, 18.3, -3.9, -4.4; IR (Neat Film, NaCl) 3348, 3078, 3042, 2929, 2893, 2855, 2737 , $2708,1687,1683,1649,1472,1463,1451,1388,1375,1360,1314,1287,1251,1218,1189,1157$, 1141, 1064, 1006, 980, 941, 868, 836, 775, 735, $677 \mathrm{~cm}^{-1}$; HRMS (FAB+) m/z calc'd for $\mathrm{C}_{15} \mathrm{H}_{19} \mathrm{O}_{3} \mathrm{~N}_{2}\left[\mathrm{M}+\mathrm{H}-\mathrm{H}_{2}\right]^{+}:$331.2093, found 331.2096; $[\alpha]_{\mathrm{D}}^{25.0}-60.8^{\circ}\left(c 0.44, \mathrm{CHCl}_{3}\right)$.

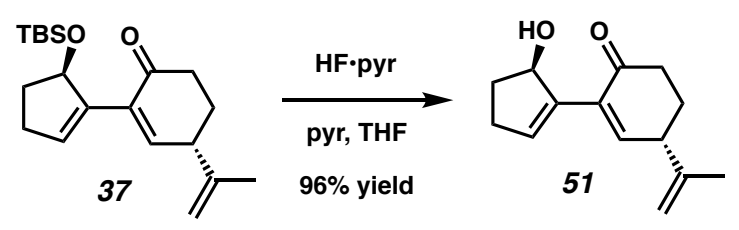

(S)-2-((R)-5-Hydroxycyclopent-1-en-1-yl)-4-(prop-1-en-2-yl)cyclohex-2-en-1-one (51): To a round-bottom plastic coated flask equipped with a magnetic stir bar were added diene 37 (30 mg, $0.090 \mathrm{mmol})$, THF (4 mL), and pyridine $(0.05 \mathrm{~mL}, 0.62 \mathrm{mmol})$. A solution of HF•pyr (pyridine $30 \%$, hydrogen fluoride $70 \%, 0.1 \mathrm{~mL}$ ) was added dropwise. The reaction mixture was stirred for $18 \mathrm{~h}$ at $23{ }^{\circ} \mathrm{C}$. The reaction was diluted with $\mathrm{Et}_{2} \mathrm{O}(4 \mathrm{~mL})$ and neutralized with sat. aq. $\mathrm{NaHCO}_{3}$ $(10 \mathrm{~mL})$. The phases were separated and the aqueous phase was extracted with EtOAc $(3 \times 10$ $\mathrm{mL}$ ). The combined organic phases were dried over $\mathrm{MgSO}_{4}$, filtered and concentrated under reduced pressure. The crude product was purified by flash column chromatography $(5: 1$, hexanes: EtOAc) to afford allylic alcohol $51(19 \mathrm{mg}, 0.087 \mathrm{mmol}, 96 \%$ yield $)$ as a colorless oil; $\mathrm{R}_{f}=0.25$ (2:1, hexanes: EtOAc); ${ }^{1} \mathrm{H}$ NMR (400 MHz, $\left.\mathrm{C}_{6} \mathrm{D}_{6}\right) \delta$ 6.86-6.76 (m, 1H), 6.44-6.35 (m, 1H), 4.99- 
$4.90(\mathrm{~m}, 1 \mathrm{H}), 4.82-4.74(\mathrm{~m}, 1 \mathrm{H}), 4.74-4.69(\mathrm{~m}, 1 \mathrm{H}), 2.96(\mathrm{~s}, 1 \mathrm{H}), 2.58(\mathrm{dt}, J=8.7,4.2 \mathrm{~Hz}, 1 \mathrm{H})$, 2.54-2.43 (m, 1H), $2.36(\mathrm{ddd}, J=16.3,6.2,4.3 \mathrm{~Hz}, 1 \mathrm{H}), 2.14-1.96(\mathrm{~m}, 3 \mathrm{H}), 1.93-1.78(\mathrm{~m}, 1 \mathrm{H})$, 1.63-1.42 (m, 5H); ${ }^{13} \mathrm{C}$ NMR (101 MHz, $\left.\mathrm{C}_{6} \mathrm{D}_{6}\right) \delta 198.9,149.3,146.2,142.2,135.2,134.0,112.4$, 77.5, 44.3, 37.9, 34.0, 30.9, 27.8, 21.1; IR (Neat Film, NaCl) 3418, 3077, 3040, 2938, 2848, 1674, $1586,1451,1377,1309,1086,1047,990,935,895 \mathrm{~cm}^{-1}$; HRMS (FAB + ) $m / z$ calc'd for $\mathrm{C}_{14} \mathrm{H}_{17} \mathrm{O}_{2}$ $\left[\mathrm{M}+\mathrm{H}-\mathrm{H}_{2}\right]^{+}:$217.1229, found $217.1235 ;[\alpha]_{\mathrm{D}}^{25.0}-120.4^{\circ}\left(c 0.33, \mathrm{CHCl}_{3}\right)$.
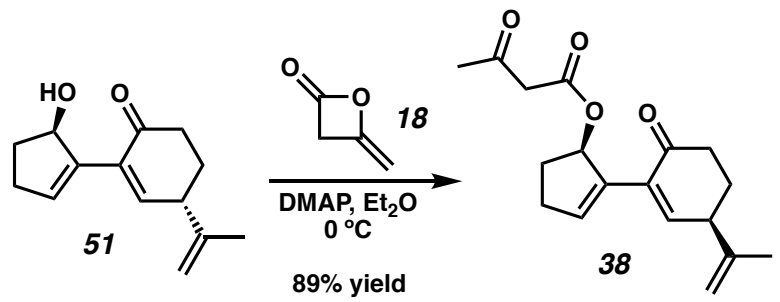

$(R)-2-((R)-6-0 x 0-3-(p r o p-1-e n-2-y l) c y c l o h e x-1-e n-1-y l) c y c l o p e n t-2-e n-1-y l$

3-

oxobutanoate (38): To a flame-dried round-bottom flask equipped with a magnetic stir bar were added allylic alcohol 51 (870 mg, $3.99 \mathrm{mmol})$, 4-dimethylaminopyridine (50 mg, $0.41 \mathrm{mmol})$ and $\mathrm{Et}_{2} \mathrm{O}(20 \mathrm{~mL})$. The flask was cooled to $0{ }^{\circ} \mathrm{C}$ and stirred for $10 \mathrm{~min}$. Diketene $(\mathbf{1 8}, 0.36 \mathrm{~mL}, 4.67$ mmol) was added dropwise. The reaction mixture stirred for $15 \mathrm{~min}$ at $0{ }^{\circ} \mathrm{C}$ was then quenched with ice-cold water ( $20 \mathrm{~mL})$. The mixture was extracted with $\mathrm{Et}_{2} \mathrm{O}(3 \times 20 \mathrm{~mL})$. The combined organic layers were washed by brine $(15 \mathrm{~mL})$, dried over $\mathrm{MgSO}_{4}$, and concentrated under reduced pressure. The crude oil was purified by flash column chromatography (4:1 hexanes: EtOAc) to afford $\beta$-ketoester 38 (1.07 $\mathrm{g}, 3.54 \mathrm{mmol}, 89 \%$ yield $)$ as a colorless oil; $\mathbf{R}_{f}=0.40(2: 1$ hexanes:Et $\left.{ }_{2} \mathrm{O}\right) ;{ }^{1} \mathrm{H}$ NMR $\left(400 \mathrm{MHz}, \mathrm{CD}_{2} \mathrm{Cl}_{2}\right) \delta$ 6.74-6.72 (m, 1H), 6.70-6.68 (m, 1H), $6.05(\mathrm{dt}, J$ $=7.5,2.4 \mathrm{~Hz}, 1 \mathrm{H}), 4.89(\mathrm{t}, J=1.5 \mathrm{~Hz}, 1 \mathrm{H}), 4.76-4.73(\mathrm{~m}, 1 \mathrm{H}), 3.40-3.33(\mathrm{~m}, 2 \mathrm{H}), 3.15(\mathrm{dt}, J=$ 8.7, 4.4 Hz, 1H), 2.65-2.27 (m, 5H), $2.18(\mathrm{~s}, 3 \mathrm{H}), 2.17-2.09(\mathrm{~m}, 1 \mathrm{H}), 1.98-1.81(\mathrm{~m}, 2 \mathrm{H}), 1.79(\mathrm{t}$, 
$J=1.2 \mathrm{~Hz}, 3 \mathrm{H}) ;{ }^{13} \mathrm{C} \mathrm{NMR}\left(101 \mathrm{MHz}, \mathrm{CD}_{2} \mathrm{Cl}_{2}\right) \delta 200.7,198.5,167.3,148.8,146.5,138.1,136.2$, 133.1, 112.3, 81.4, 50.6, 44.4, 38.1, 31.7, 30.8, 30.3, 28.0, 21.4; IR (Neat Film, NaCl) 3655, 3643, $3080,2943,2850,1726,1640,1554,1450,1356,1315,1256,1146,1088,1029,995,900,854$, 778, 706, 634, $617 \mathrm{~cm}^{-1}$; HRMS (FAB+) m/z calc'd for $\mathrm{C}_{18} \mathrm{H}_{23} \mathrm{O}_{4}[\mathrm{M}+\mathrm{H}]^{+}:$303.1596, found 303.1594; $[\alpha]_{\mathrm{D}}^{25.0}-30.6^{\circ}\left(c 0.13, \mathrm{CHCl}_{3}\right)$. (Note: the enol ether tautomer of $\beta$-ketoester 38 was predominant in $\mathrm{CD}_{2} \mathrm{Cl}_{2}$.
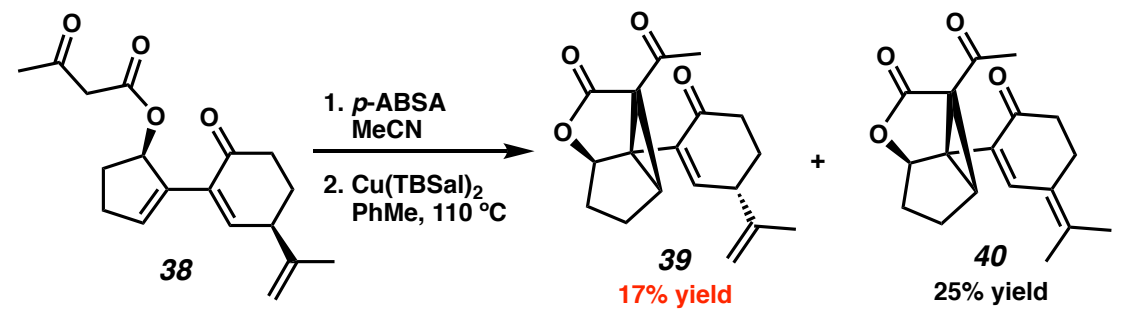

\section{$\left(2 \mathrm{a} S, 2 \mathrm{a}^{1} S, 4 \mathrm{a} R\right)-2 \mathrm{~b}-\mathrm{Acetyl}-2 \mathrm{a}^{1}-((S)-6-0 x 0-3-($ prop-1-en-2-yl)cyclohex-1-en-1-}

yl)hexahydro-3H-4-oxacyclopropa[cd]pentalen-3-one (39): To a round-bottom flask equipped with a magnetic stir bar were added $\beta$-ketoester 38 (95 mg, $0.314 \mathrm{mmol})$, MeCN (3 mL), and $p$ ABSA (113 mg, $0.47 \mathrm{mmol}) . \mathrm{Et}_{3} \mathrm{~N}(0.1 \mathrm{~mL}, 0.717 \mathrm{mmol})$ was added dropwise. The reaction mixture was remained to stir $2 \mathrm{~h}$ at $23^{\circ} \mathrm{C}$. The reaction mixture was filtered through a Florisil (2:1, pentanes: $\mathrm{Et}_{2} \mathrm{O}$ ) was then concentrated under reduced pressure. The residue was used in the next reaction without further purification.

To a flame-dried two neck round-bottom flask equipped with a magnetic stir bar was added $\mathrm{Cu}(\mathrm{TBSal})_{2}(8 \mathrm{mg}, 0.019 \mathrm{mmol})$ in a nitrogen-filled glove box. The flask was sealed with rubber septa and removed from the glove box. One of the rubber septa was replaced with a reflux condenser connected to a nitrogen inlet. A solution of semi-crude diazo ester (60 mg, $0.198 \mathrm{mmol})$ in toluene $(40 \mathrm{~mL})$ was added. The reaction was heated to reflux in a $110^{\circ} \mathrm{C}$ oil bath. After $3 \mathrm{~h}$ of 
stirring, the reaction mixture was cooled to $23{ }^{\circ} \mathrm{C}$ and stirred for $15 \mathrm{~min}$. The mixture was concentrated and purified by flash column chromatography (10:1 hexanes: EtOAc) to afford cyclopropane 39 (10 mg, $0.033 \mathrm{mmol}, 17 \%$ yield $)$ as a colorless oil; $\mathrm{R}_{f}=0.40(2: 1$ hexanes:EtOAc); ${ }^{1} \mathrm{H}$ NMR $\left(400 \mathrm{MHz}, \mathrm{CDCl}_{3}\right) \delta 6.79(\mathrm{dd}, J=3.2,1.1 \mathrm{~Hz}, 1 \mathrm{H}), 4.96-4.89(\mathrm{~m}, 1 \mathrm{H})$, 4.75-4.73 (m, 1H), 4.73-4.71 (m, 1H), $3.13(\mathrm{dt}, J=8.3,4.2 \mathrm{~Hz}, 1 \mathrm{H}), 2.96(\mathrm{dd}, J=6.5,1.0 \mathrm{~Hz}$, 1H), $2.56(\mathrm{ddd}, J=16.8,6.5,4.4 \mathrm{~Hz}, 1 \mathrm{H}), 2.44(\mathrm{~s}, 3 \mathrm{H}), 2.40-2.26(\mathrm{~m}, 2 \mathrm{H}), 2.21-2.00(\mathrm{~m}, 2 \mathrm{H})$, 2.00-1.78 (m, 6H); $\left.{ }^{13} \mathrm{C} \mathrm{NMR} \mathrm{(101} \mathrm{MHz}, \mathrm{CDCl}_{3}\right) \delta 198.5,198.2,172.3,153.8,145.2,131.7,112.9$, 85.7, 77.2, 59.2, 50.7, 43.7, 38.9, 38.6, 36.5, 29.9, 27.7, 23.9, 21.7; IR (Neat Film, NaCl) 3371, $3077,2939,1760,182,1651,1488,1439,1362,1339,1309,1242,1223,1190,1160,1136,1085$, $1067,1006,957,912,850,817,727,703,622,612 \mathrm{~cm}^{-1}$; HRMS (MM+) $m / z$ calc'd for $\mathrm{C}_{15} \mathrm{H}_{19} \mathrm{O}_{3}$ $[\mathrm{M}+\mathrm{H}]^{+}: 301.1440$, found $301.1450 ;[\alpha]_{\mathrm{D}}^{25.0}-56.8^{\circ}\left(c 0.30, \mathrm{CHCl}_{3}\right)$, and side product $40(15 \mathrm{mg}$, $0.050 \mathrm{mmol}, 25 \%$ yield) as a colorless oil; $\mathrm{R}_{f}=0.05$ (2:1 hexanes: EtOAc); ${ }^{1} \mathrm{H}$ NMR $(400 \mathrm{MHz}$, $\left.\mathrm{CDCl}_{3}\right) \delta 7.32(\mathrm{~s}, 1 \mathrm{H}), 4.75(\mathrm{dd}, J=2.0,1.1 \mathrm{~Hz}, 1 \mathrm{H}), 3.03(\mathrm{dt}, J=6.5,1.1 \mathrm{~Hz}, 1 \mathrm{H}), 2.75-2.60(\mathrm{~m}$, 2H), 2.54-2.35 (m, 6H), 2.10-2.01 (m, 1H), 2.01-1.96 (m, 3H), 1.96-1.84 (m, 5H); ${ }^{13} \mathrm{C}$ NMR (101 $\left.\mathrm{MHz}, \mathrm{CDCl}_{3}\right) \delta 198.6,198.2,172.5,144.5,142.3,126.3,126.1,85.8,77.2,60.1,51.5,38.5,38.4$, 37.1, 29.8, 25.6, 23.9, 22.2, 21.3; IR (Neat Film, NaCl) 3484, 3369, 3051, 2928, 2853, 2435, 2305, $2143,1755,1679,1615,1434,1361,1348,1311,1297,1257,1242,1216,1199,1164,1131,1090$, $1064,1037,1004,966,918,888,851,822,798,753,719,667,655,633,614 \mathrm{~cm}^{-1}$; HRMS(FAB+) $m / z$ calc'd for $\mathrm{C}_{18} \mathrm{H}_{21} \mathrm{O}_{4}[\mathrm{M}+\mathrm{H}]^{+}:$301.1440, found 301.1434; $[\alpha]_{\mathrm{D}}^{25.0} 53.1^{\circ}\left(c 0.10, \mathrm{CHCl}_{3}\right)$ 


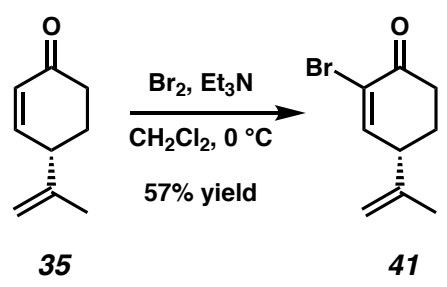

(S)-2-Bromo-4-(prop-1-en-2-yl)cyclohex-2-en-1-one (41): To a flame-dried round-bottom flask equipped with a magnetic stir bar were added ketone 35 (553 mg, $4.06 \mathrm{mmol}$ ) and DCM (35 $\mathrm{mL})$. The flask was cooled to $0{ }^{\circ} \mathrm{C}$ and stirred for $10 \mathrm{~min}$. A solution of bromine $(0.24 \mathrm{~mL}, 4.66$ $\mathrm{mmol})$ in DCM (5 mL) was added dropwise with vigorous stirring at $0{ }^{\circ} \mathrm{C}$. After reaction became a reddish-brown color, $\mathrm{Et}_{3} \mathrm{~N}(0.6 \mathrm{~mL}, 4.30 \mathrm{mmol})$ was added at $0{ }^{\circ} \mathrm{C}$. The cooling bath was removed, and the flask was allowed to warm to $23{ }^{\circ} \mathrm{C}$. After $30 \mathrm{~min}$ of stirring, the reaction was washed with water $(40 \mathrm{~mL})$. The aqueous phase was extracted with DCM (3 x $40 \mathrm{~mL})$. The combined organic phases were dried over $\mathrm{MgSO}_{4}$, filtered, and concentrated under reduced pressure. The crude product was purified by flash column chromatography (20:1 hexanes: EtOAc) to afford bromide 41 as a yellow oil (500 mg, $2.32 \mathrm{mmol}, 57 \%$ yield); $\mathrm{R}_{f}=0.45$ (6:1 hexanes: EtOAc); ${ }^{1} \mathrm{H}$ NMR $\left(500 \mathrm{MHz}, \mathrm{CDCl}_{3}\right) \delta 7.29(\mathrm{dd}, J=3.6,0.9 \mathrm{~Hz}, 1 \mathrm{H}), 4.96-4.88(\mathrm{~m}, 1 \mathrm{H}), 4.87-$ $4.72(\mathrm{~m}, 1 \mathrm{H}), 3.19-3.08(\mathrm{~m}, 1 \mathrm{H}), 2.70(\mathrm{ddd}, J=16.6,7.0,4.3 \mathrm{~Hz}, 1 \mathrm{H}), 2.51(\mathrm{ddd}, J=16.6,10.7$, $4.5 \mathrm{~Hz}, 1 \mathrm{H}), 2.19(\mathrm{ddtd}, J=12.8,7.0,4.7,1.0 \mathrm{~Hz}, 1 \mathrm{H}), 1.99(\mathrm{dddd}, J=13.5,10.7,8.2,4.4 \mathrm{~Hz}$, 1H), $1.79(\mathrm{dd}, J=1.1 \mathrm{~Hz}, 3 \mathrm{H}) ;{ }^{13} \mathrm{C} \mathrm{NMR}\left(126 \mathrm{MHz}, \mathrm{CDCl}_{3}\right) \delta 191.2,153.1,144.2,124.0,113.4$, 46.1, 36.5, 27.6, 21.4.; IR (Neat Film, NaCl) 3853, 3650, 3371, 3035, 2953, 2869, 2360, 1694, $1646,1595,1451,1417,1377,1327,1278,1218,1172,1153,1132,1085,1037,984,958,899$, 816, 798, 786, 749, 716, 668, 650, $611 \mathrm{~cm}^{1}$; HRMS $(\mathrm{FAB}+) \mathrm{m} / z$ calc'd for $\mathrm{C}_{9} \mathrm{H}_{12} \mathrm{OBr}[\mathrm{M}+\mathrm{H}]^{+}$: 215.0072 , found $215.0071 ;[\alpha]_{\mathrm{D}}^{25.0} 52.9^{\circ}\left(c 0.30, \mathrm{CHCl}_{3}\right)$. 


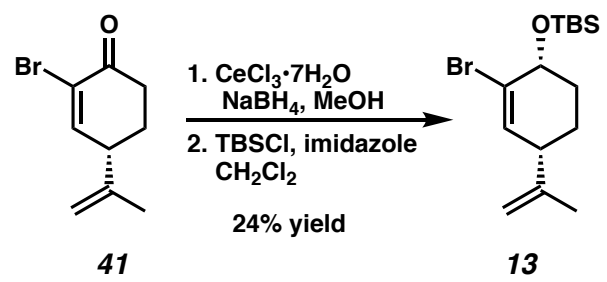

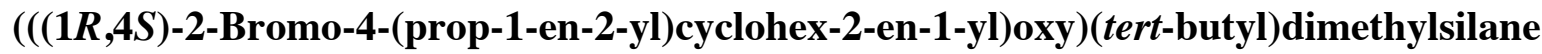

(13): To a round-bottom flask equipped with a magnetic stir bar were added bromoenone 41 (7.68 $\mathrm{g}, 35.7 \mathrm{mmol})$ and $\mathrm{MeOH}(108 \mathrm{~mL})$. The flask was cooled to $0{ }^{\circ} \mathrm{C}$, after which $\mathrm{CeCl}_{3} \bullet 7 \mathrm{H}_{2} \mathrm{O}(13.3$ g 35.7 mmol, 1.0 equiv) and $\mathrm{NaBH}_{4}(1.35 \mathrm{~g}, 35.7 \mathrm{mmol}, 1.0$ equiv) were sequentially added over $5 \mathrm{~min}$. The reaction was stirred at $0{ }^{\circ} \mathrm{C}$ for $20 \mathrm{~min}$, and the mixture was poured into sat. aq. $\mathrm{NH}_{4} \mathrm{Cl}$ $(300 \mathrm{~mL})$. The aqueous phase was extracted with $\mathrm{Et}_{2} \mathrm{O}(3 \times 200 \mathrm{~mL})$. The combined organic phases were dried over $\mathrm{MgSO}_{4}$, filtered, and concentrated under reduced pressure. The crude product was passed through a plug of silica (20\% EtOAc in hexanes) to afford crude alcohol as a colorless oil $(7.01 \mathrm{~g})$.

The semi-crude residue was dissolved in $\mathrm{CH}_{2} \mathrm{Cl}_{2}(81 \mathrm{~mL})$, and imidazole $(5.1 \mathrm{~g}, 74.3 \mathrm{mmol}$, 2.3 equiv $)$ and $\mathrm{TBSCl}(8.3 \mathrm{~g}, 54.9 \mathrm{mmol}, 1.7$ equiv $)$ were sequentially added. The resulting mixture was stirred at $23{ }^{\circ} \mathrm{C}$ for $12 \mathrm{~h}$, after which it was poured into brine $(200 \mathrm{~mL})$, extracted with $\mathrm{CH}_{2} \mathrm{Cl}_{2}$ (3 x $200 \mathrm{~mL}$ ) dried over $\mathrm{MgSO}_{4}$. The crude solution was concentrated in vacuo and purified by flash column chromatography (1\% to 5\% EtOAc in hexanes) to afford bromide $\mathbf{1 3}$ as a colorless oil (2.85 g, mmol, 24\% yield); $\mathrm{R}_{f}=0.90$ (6:1 hexanes: EtOAc); ${ }^{1} \mathrm{H}$ NMR $\left(500 \mathrm{MHz}, \mathrm{CDCl}_{3}\right) \delta 6.03$ $(\mathrm{dd}, J=2.9,0.8 \mathrm{~Hz}, 1 \mathrm{H}), 4.81-4.75(\mathrm{~m}, 2 \mathrm{H}), 4.18(\mathrm{td}, J=3.7,1.2 \mathrm{~Hz}, 1 \mathrm{H}), 2.79-2.70(\mathrm{~m}, 1 \mathrm{H})$, $1.88-1.83(\mathrm{~m}, 1 \mathrm{H}), 1.79-1.73(\mathrm{~m}, 1 \mathrm{H}), 1.73-1.71(\mathrm{~m}, 4 \mathrm{H}), 1.68-1.62(\mathrm{~m}, 1 \mathrm{H}), 0.91(\mathrm{~s}, 9 \mathrm{H}), 0.16$ (s, 3H), $0.10(\mathrm{~s}, 3 \mathrm{H}) ;{ }^{13} \mathrm{C}$ NMR $\left(126 \mathrm{MHz}, \mathrm{CDCl}_{3}\right) \delta 147.1,134.5,126.3,111.5,70.6,46.7,32.7$, 26.0, 22.2, 20.6, 18.3, -4.3, -4.5; IR (Neat Film, NaCl) 3077, 2950, 2929, 2885, 2856, 2738, 2709, $2360,1918,1793,2738,2709,2360,1918,1793,1684,1648,1472,1462,1448,1436,1407,1388$, 
$1375,1361,1300,1280,1251,1219,1194,1171,1126,1084,1064,1025,1006,987,960,939$, 914, 894, 880, 834, 814, 775, 729, 669, $639 \mathrm{~cm}^{-1}$; HRMS (MM+) $m / z$ calc'd for $\mathrm{C}_{15} \mathrm{H}_{19} \mathrm{O}_{3}[\mathrm{M}+\mathrm{H}-$ $\left.\mathrm{H}_{2}\right]^{+}:$331.0916, found 331.0902; $[\alpha]_{\mathrm{D}}^{25.0}-22.6^{\circ}\left(c 0.30, \mathrm{CHCl}_{3}\right)$.

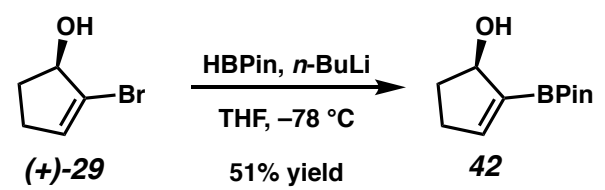

(R)-2-(4,4,5,5-Tetramethyl-1,3,2-dioxaborolan-2-yl)cyclopent-2-en-1-ol (42): To a roundbottom flask equipped with a magnetic stir bar was added (+)-29 (326.0 mg, $2.00 \mathrm{mmol})$ and THF $(40 \mathrm{~mL})$. The resulting solution was cooled to $-78{ }^{\circ} \mathrm{C}$, and $n$-BuLi $(2.3 \mathrm{M}$ in hexanes, $4.60 \mathrm{mmol}$, $2.1 \mathrm{~mL}, 2.3$ equiv) was added dropwise over several min. The resulting suspension was stirred vigorously for $15 \mathrm{~min}$, and neat pinacolborane $(0.80 \mathrm{~mL}, 5.00 \mathrm{mmol}, 2.5$ equiv $)$ was added in one portion. The mixture was stirred vigorously for an additional $20 \mathrm{~min}$, after which it was poured into sat. aq. $\mathrm{NH}_{4} \mathrm{Cl}$, extracted with $\mathrm{Et}_{2} \mathrm{O}(3 \times 50 \mathrm{~mL})$, dried over $\mathrm{Na}_{2} \mathrm{SO}_{4}$, and concentrated in vасио. The crude product was purified by flash column chromatography (4:1, hexanes: EtOAc) to afford boronate 42 (213.7 $\mathrm{mg}, 1.01 \mathrm{mmol}, 51 \%$ yield $)$ as a white solid; $\mathrm{R}_{f}=0.10$ (6:1, hexanes: EtOAc); ${ }^{1} \mathrm{H}$ NMR $\left(400 \mathrm{MHz}, \mathrm{CDCl}_{3}\right) \delta$ 6.70-6.63 (m, 1H), 5.05-4.95 (m, 1H), 2.64-2.51 (m, 1H), 2.41-2.18 (m, 2H), 1.71 (dddd, $J=13.7,9.1,5.5,4.5 \mathrm{~Hz}, 1 \mathrm{H}), 1.28(\mathrm{~s}, 12 \mathrm{H}) ;{ }^{13} \mathrm{C} \mathrm{NMR}(126 \mathrm{MHz}$, $\left.\mathrm{CDCl}_{3}\right) \delta 150.1,83.6,79.8,33.2,33.0,26.0,25.0$; IR (Neat Film, NaCl) 3478, 3038, 2978, 2931, $2731,2219,1995,1887,1622,1615,1372,1214,1144,1111,1046,1020,964,925,854,832$, 759, $710 \mathrm{~cm}^{-1}$; HRMS (FAB+) $m / z$ calc'd for $\mathrm{C}_{15} \mathrm{H}_{19} \mathrm{O}_{3} \mathrm{~N}_{2}\left[\mathrm{M}+\mathrm{H}-\mathrm{H}_{2}\right]^{+}:$209.1349, found 209.1344; $[\alpha]_{\mathrm{D}}^{25.0}-59.6^{\circ}\left(c 0.80, \mathrm{CHCl}_{3}\right)$. 


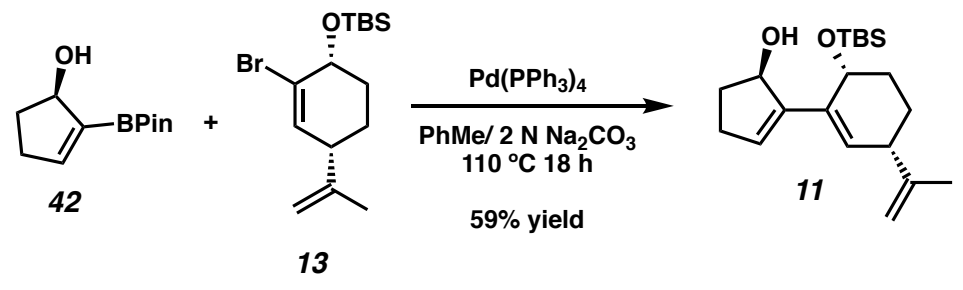

\section{(R)-2-((3S,6R)-6-((tert-Butyldimethylsilyl)oxy)-3-(prop-1-en-2-yl)cyclohex-1-en-1-}

yl)cyclopent-2-en-1-ol (11): To a two neck round-bottom flask equipped with reflux condenser and a magnetic stir bar were added boronate $42(200 \mathrm{mg}, 0.952 \mathrm{mmol})$ and bromide 13 (200 mg, $0.605 \mathrm{mmol})$. The mixture was evacuated and back-filled with argon (x3). Toluene $(6 \mathrm{~mL})$, tetrakis(triphenylphosphine)palladium(0) (21 mg, $0.018 \mathrm{mmol})$, and $2 \mathrm{M}$ aqueous $\mathrm{Na}_{2} \mathrm{CO}_{3}(6 \mathrm{~mL})$ were added. The reaction was heated to reflux in a $110{ }^{\circ} \mathrm{C}$ oil bath. After $18 \mathrm{~h}$ of stirring, the reaction mixture was cooled to $23{ }^{\circ} \mathrm{C}$ and stirred for $15 \mathrm{~min}$. The phases were separated and the aqueous phases were extracted with EtOAc $(3 \times 10 \mathrm{~mL})$. The combined organic phases were washed with brine $(10 \mathrm{~mL})$, dried over $\mathrm{MgSO}_{4}$, filtered and concentrated under reduced pressure. The crude product was purified by flash column chromatography (20:1 hexanes: EtOAc) to afford diene 11 (120 mg, $0.359 \mathrm{mmol}, 59 \%$ yield) as a colorless oil; $\mathrm{R}_{f}=0.40$ (6:1, hexanes: EtOAc); ${ }^{1} \mathrm{H}$ $\operatorname{NMR}\left(500 \mathrm{MHz}, \mathrm{CDCl}_{3}\right) \delta 5.85-5.81(\mathrm{~m}, 2 \mathrm{H}), 4.95(\mathrm{dt}, J=7.2,2.5 \mathrm{~Hz}, 1 \mathrm{H}), 4.80-4.78(\mathrm{~m}, 1 \mathrm{H})$, $4.77(\mathrm{dd}, J=2.0,1.4 \mathrm{~Hz}, 1 \mathrm{H}), 4.43(\mathrm{ddd}, J=3.6,2.8,1.3 \mathrm{~Hz}, 1 \mathrm{H}), 2.85-2.78(\mathrm{~m}, 1 \mathrm{H}), 2.62-2.50$ (m, 1H), 2.38-2.28 (m, 1H), 2.26-2.16 (m, 1H), 1.93-1.80 (m, 2H), 1.80-1.74 (m, 1H), $1.72(\mathrm{dd}$ $J=1.5,0.8 \mathrm{~Hz}, 3 \mathrm{H}), 1.68-1.58(\mathrm{~m}, 2 \mathrm{H}), 0.85(\mathrm{~s}, 9 \mathrm{H}), 0.09(\mathrm{~s}, 3 \mathrm{H}), 0.05(\mathrm{~s}, 3 \mathrm{H}) ;{ }^{13} \mathrm{C}$ NMR $(126$ $\left.\mathrm{MHz}, \mathrm{CDCl}_{3}\right) \delta 149.0,145.1,135.0,130.7,128.7,110.9,76.8,65.1,44.9,33.7,31.8,30.7,26.0$, 22.4, 20.5, 18.3,-3.9, -4.2; IR (Neat Film, NaCl) 3601, 3412, 3072, 2929, 2855, 2737, 2708, 1924 , $1647,1472,1463,1436,1407,1389,1375,1360,1334,1305,1252,1218,1024,959,934,889$, 
835, 773, 723, $676 \mathrm{~cm}^{-1}$; HRMS (MM+) m/z calc'd for $\mathrm{C}_{20} \mathrm{H}_{34} \mathrm{O}_{2} \mathrm{NSiNa}[\mathrm{M}+\mathrm{Na}]^{+}:$356.2220, found $357.2237 ;[\alpha]_{\mathrm{D}}^{25.0}-21.1^{\circ}\left(c 0.10, \mathrm{CHCl}_{3}\right)$.
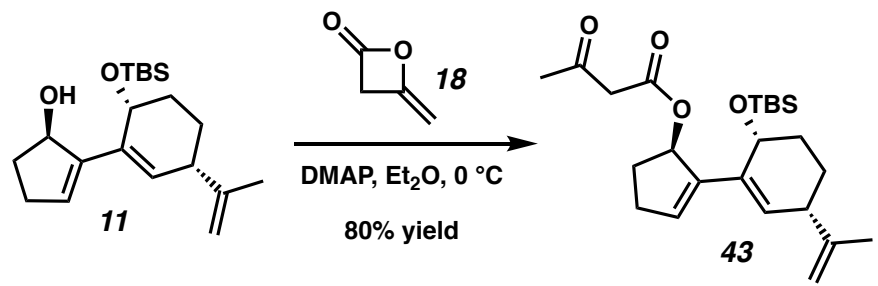

\section{(R)-2-((3S,6R)-6-((tert-Butyldimethylsilyl)oxy)-3-(prop-1-en-2-yl)cyclohex-1-en-1-}

yl)cyclopent-2-en-1-yl 3-oxobutanoate (43): To a two neck round-bottom flask with a magnetic stir bar and were added bicyclic alcohol 11 (20 mg, $0.060 \mathrm{mmol})$, 4-dimethylaminopyridine (1.0 $\mathrm{mg}, 0.0082 \mathrm{mmol})$ and $\mathrm{Et}_{2} \mathrm{O}(1.5 \mathrm{~mL})$. The flask was cooled to $0{ }^{\circ} \mathrm{C}$ and stirred for $10 \mathrm{~min}$. A solution of diketene $(\mathbf{1 8}, 0.07 \mathrm{~mL}, 0.907 \mathrm{mmol})$ in $\mathrm{Et}_{2} \mathrm{O}(2 \mathrm{~mL})$ was added dropwise over several $\min$. The reaction mixture was stirred for $15 \mathrm{~min}$ at $0{ }^{\circ} \mathrm{C}$ was then quenched by ice cold water $(2$ $\mathrm{mL})$. The mixture was extracted with $\mathrm{Et}_{2} \mathrm{O}(3 \times 3 \mathrm{~mL})$. The combined organic layers were washed with brine $(3 \mathrm{~mL})$, dried over $\mathrm{MgSO}_{4}$, and concentrated under reduced pressure. The crude oil was purified by flash column chromatography (4:1 hexanes: EtOAc) to afford $\beta$-ketoester $\mathbf{4 3}$ (20 mg, $0.048 \mathrm{mmol}, 80 \%$ yield) as a colorless oil; $\mathrm{R}_{f}=0.45$ (6:1, hexanes: $\left.\mathrm{Et}_{2} \mathrm{O}\right) ;{ }^{1} \mathrm{H} \mathrm{NMR}(500 \mathrm{MHz}$, $\left.\mathrm{CDCl}_{3}\right) \delta 6.18-5.98(\mathrm{~m}, 2 \mathrm{H}), 5.62(\mathrm{~d}, J=2.8 \mathrm{~Hz}, 1 \mathrm{H}), 4.85-4.67(\mathrm{~m}, 2 \mathrm{H}), 4.44(\mathrm{t}, J=3.2 \mathrm{~Hz}, 1 \mathrm{H})$, $3.36(\mathrm{~s}, 2 \mathrm{H}), 2.77(\mathrm{t}, J=8.6 \mathrm{~Hz}, 1 \mathrm{H}), 2.62-2.53(\mathrm{~m}, 1 \mathrm{H}), 2.44-2.27(\mathrm{~m}, 2 \mathrm{H}), 2.22(\mathrm{~s}, 3 \mathrm{H}), 1.96-$ $1.83(\mathrm{~m}, 2 \mathrm{H}), 1.79-1.72(\mathrm{~m}, 1 \mathrm{H}), 1.73-1.54(\mathrm{~m}, 5 \mathrm{H}), 0.84(\mathrm{~s}, 9 \mathrm{H}), 0.08(\mathrm{~s}, 3 \mathrm{H}), 0.05(\mathrm{~s}, 3 \mathrm{H}) ;{ }^{13} \mathrm{C}$ NMR (126 MHz, $\left.\mathrm{CDCl}_{3}\right) \delta 200.8,167.3,148.8,140.9,134.4,131.8,130.4,110.6,79.8,64.7,50.4$, 44.7, 31.7, 31.1, 30.8, 30.3, 25.9, 22.3, 20.4, 18.2, -3.8, -4.4; IR (Neat Film, NaCl) 2976, 2926, $2854,1876,1659,1612,1584,1512,1464,1410,1388,1379,1370,1315,1246,1175,1166,1145$, 
1113, 1039, 967, 862, 819, 750, 688, $671 \mathrm{~cm}^{-1}$; HRMS (MM+) m/z calc'd for $\mathrm{C}_{24} \mathrm{H}_{38} \mathrm{O}_{4} \mathrm{SiNa}$ $[\mathrm{M}+\mathrm{Na}]^{+}:$441.2432, found 441.2441; $[\alpha]_{\mathrm{D}}^{25.0} 4.4^{\circ}\left(c 0.34, \mathrm{CHCl}_{3}\right)$.

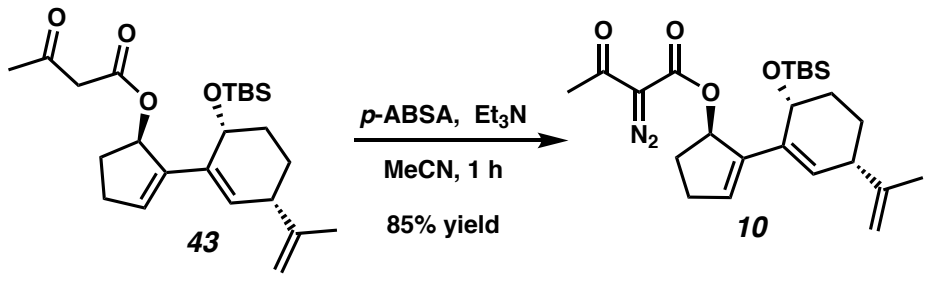

\section{(R)-2-((3S,6R)-6-((tert-Butyldimethylsilyl)oxy)-3-(prop-1-en-2-yl)cyclohex-1-en-1-}

yl)cyclopent-2-en-1-yl 2-diazo-3-oxobutanoate (10): To a round-bottom flask equipped with a magnetic stir bar were added $\beta$-ketoester 43 (20 mg, $0.048 \mathrm{mmol})$, MeCN (2.5 mL), and $p$-ABSA (40.0 mg, $0.167 \mathrm{mmol}) . \mathrm{Et}_{3} \mathrm{~N}(0.03 \mathrm{~mL}, 0.215 \mathrm{mmol})$ was added dropwise. The reaction mixture was stirred for $1 \mathrm{~h} \mathrm{~min}$ at $23{ }^{\circ} \mathrm{C}$ and concentrated in vacuo. The resulting residue was passed through a silica gel plug $\left(4: 1\right.$ pentane: $\left.\mathrm{Et}_{2} \mathrm{O}\right)$ and concentrated under reduced pressure to afford diazo ester 10 (18 mg, $0.041 \mathrm{mmol}, 85 \%$ yield) as a yellowish oil; $\mathrm{R}_{f}=0.44$ (4:1 hexanes: EtOAc); ${ }^{1} \mathrm{H}$ NMR $\left(500 \mathrm{MHz}, \mathrm{CDCl}_{3}\right) \delta 6.08\left(\mathrm{dt}, J=1.66 \mathrm{~Hz}, 1.66 \mathrm{~Hz}, 7.75 \mathrm{~Hz}, 1 \mathrm{H} ;{ }^{13} \mathrm{C} \mathrm{NMR}(126 \mathrm{MHz}\right.$, $\left.\mathrm{CDCl}_{3}\right) \delta$ 190.47; IR (Neat Film, NaCl) 3408, 3073, 2929, 2855, 2362, 2139, 1713, 1661, 1652, $1472,1464,1366,1312,1250,1195,1150,1086,1064,1025,1006,963,938,921,895,850,834$, 808, 773, 742, 676, $635 \mathrm{~cm}^{-1}$; HRMS (MM+) m/z calc'd for $\mathrm{C}_{24} \mathrm{H}_{36} \mathrm{O}_{4} \mathrm{~N}_{2} \mathrm{SiNa}[\mathrm{M}+\mathrm{Na}]^{+}: 467.2337$, found $467.2354 ;[\alpha]_{\mathrm{D}}^{25.0}-11.4^{\circ}\left(c 0.31, \mathrm{CHCl}_{3}\right)$.

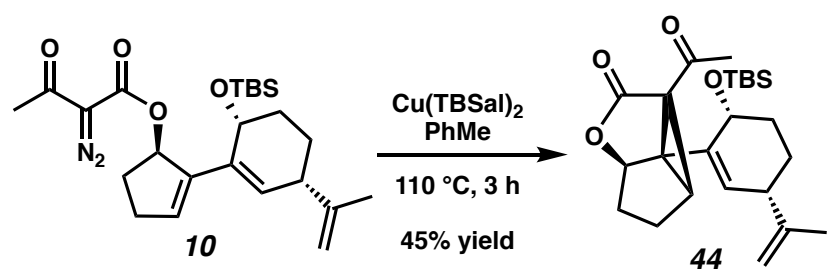


$\left(2 \mathrm{a} S, 2 \mathrm{a}^{1} S, 4 \mathrm{a} R\right)-2 \mathrm{~b}-A c e t y l-2 \mathrm{a}^{1}-((3 S, 6 R)-6$-((tert-butyldimethylsilyl)oxy)-3-(prop-1-en-2yl)cyclohex-1-en-1-yl)hexahydro-3H-4-oxacyclopropa[cd]pentalen-3-one (44): To a flamedried two neck round-bottom flask equipped with a magnetic stir bar was added $\mathrm{Cu}(\mathrm{TBSal})_{2}(3.0$ $\mathrm{mg}, 0.0072 \mathrm{mmol}$ ) in a nitrogen-filled glove box. The flask was sealed with rubber septa and removed from the glove box. One of the rubber septa was replaced with a reflux condenser connected to a nitrogen inlet. A solution of diazo ester $\mathbf{1 0}(20 \mathrm{mg}, 0.045 \mathrm{mmol})$ in toluene $(15 \mathrm{~mL})$ was added. The reaction was heated to reflux in a $110^{\circ} \mathrm{C}$ oil bath. After $3 \mathrm{~h}$ of stirring, the reaction mixture was cooled to $23{ }^{\circ} \mathrm{C}$ and stirred for $15 \mathrm{~min}$. The mixture was concentrated and purified by flash column chromatography (10:1 hexanes, EtOAc) to afford cyclopropane 44 (8.4 mg, 0.020 mmol, $45 \%$ yield) as a white solid; $\mathrm{R}_{f}=0.40$ (6:1 hexanes: EtOAc); ${ }^{1} \mathrm{H} \mathrm{NMR}\left(500 \mathrm{MHz}, \mathrm{CDCl}_{3}\right)$ $\delta 5.69(\mathrm{~d}, J=3.0 \mathrm{~Hz}, 1 \mathrm{H}), 5.09-5.00(\mathrm{~m}, 1 \mathrm{H}), 4.81(\mathrm{t}, J=1.7 \mathrm{~Hz}, 1 \mathrm{H}), 4.75-4.67(\mathrm{~m}, 1 \mathrm{H}), 3.84$ $3.74(\mathrm{~m}, 1 \mathrm{H}), 2.96(\mathrm{dt}, J=6.3,1.1 \mathrm{~Hz}, 1 \mathrm{H}), 2.76(\mathrm{~d}, J=7.6 \mathrm{~Hz}, 1 \mathrm{H}), 2.55(\mathrm{~s}, 3 \mathrm{H}), 2.36-2.26(\mathrm{~m}$, $1 \mathrm{H}), 2.02(\mathrm{dd}, J=13.0,5.8 \mathrm{~Hz}, 1 \mathrm{H}), 1.96-1.85(\mathrm{~m}, 1 \mathrm{H}), 1.82-1.70(\mathrm{~m}, 5 \mathrm{H}), 1.69-1.52(\mathrm{~m}, 3 \mathrm{H})$, 0.90 (s, 9H), 0.09 (s, 6H); ${ }^{13} \mathrm{C}$ NMR (126 MHz, $\left.\mathrm{CDCl}_{3}\right) \delta 198.4,172.7,147.7,136.2,132.9,111.5$, 86.4, 68.9, 65.1 , 50.6, 43.7, 42.7, 38.3, 31.0, 30.4, 26.1 , 23.9, 22.8, 21.0, 18.1, -3.8, -4.3; IR (Neat Film, NaCl) 2930, 2857, 1760, 1964, 1436, 1360, 1346, 1312, 1259, 1157, 1084, 1055, 1027, 1005, 983, 935, 896, 863, 832, 802, $774 \mathrm{~cm}^{-1}$; HRMS (EI+) $m / z$ calc'd for $\mathrm{C}_{24} \mathrm{H}_{36} \mathrm{O}_{4} \mathrm{Si}[\mathrm{M} \bullet]^{+}:$416.2383, found, 416.2379; $[\alpha]_{\mathrm{D}}^{25.0}-68.1^{\circ}\left(c 0.10, \mathrm{CHCl}_{3}\right)$. 


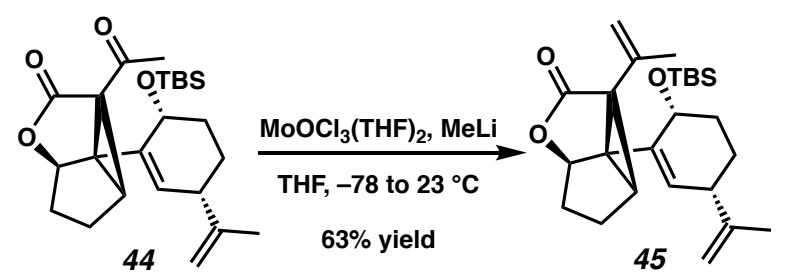

$\left(2 \mathrm{a} S, 2 \mathrm{a}^{1} S, 4 \mathrm{a} R\right)-2 \mathrm{a}^{1}-((3 S, 6 R)-6$-((tert-Butyldimethylsilyl)oxy)-3-(prop-1-en-2-yl)cyclohex1-en-1-yl)-2b-(prop-1-en-2-yl)hexahydro-3H-4-oxacyclopropa[cd]pentalen-3-one (45): Tо a flame-dried round-bottom flask equipped with a magnetic stir bar was added trichlorobis(THF) molybdenum(III) $(750 \mathrm{mg}, 2.08 \mathrm{mmol})$ in a nitrogen-filled glove box. The flask was sealed with a rubber septum, removed from the glove box and connected to a nitrogen inlet. THF ( $3 \mathrm{~mL}$ ) was added to the flask to generate a bright green solution. The flask was cooled to $-78{ }^{\circ} \mathrm{C}$ and stirred for $10 \mathrm{~min}$. A solution of $\mathrm{MeLi}\left(1.6 \mathrm{M}\right.$ in $\left.\mathrm{Et}_{2} \mathrm{O}, 1.2 \mathrm{~mL}, 1.92 \mathrm{mmol}\right)$ was added dropwise to the reaction, resulting in a dark red solution. After $1 \mathrm{~h}$ of stirring at $-78^{\circ} \mathrm{C}$, a solution of cyclopropane 44 (48 mg, $0.115 \mathrm{mmol})$ in THF (1 mL) was added dropwise. The reaction was allowed to warm to ambient temperature and stirred for an additional $6 \mathrm{~h}$. The reaction was quenched by addition of water $(4 \mathrm{~mL})$. The phases were separated, and the aqueous phase was extracted with $\mathrm{Et}_{2} \mathrm{O}(3 \mathrm{x}$ $4 \mathrm{~mL}$ ). The combined organic phases were dried over $\mathrm{MgSO}_{4}$, filtered, and concentrated under reduced pressure. The residue was purified by flash column chromatography (15:1 hexanes: EtOAc) to afford vinyl lactone $45(30 \mathrm{mg}, 0.0723 \mathrm{mmol}, 63 \%$ yield $)$ as a colorless oil; $\mathrm{R}_{f}=0.50$ (6:1 hexanes: EtOAc); ${ }^{H} \mathrm{H} \mathrm{NMR}\left(500 \mathrm{MHz}, \mathrm{CDCl}_{3}\right) \delta 5.51(\mathrm{dd}, J=2.8,0.9 \mathrm{~Hz}, 1 \mathrm{H}), 5.18-5.15(\mathrm{~m}$, 1H), 5.12-5.07 (m, 1H), 5.00-4.96 (m, 1H), $4.79(\mathrm{dd}, J=2.0,1.4 \mathrm{~Hz}, 1 \mathrm{H}), 4.73(\mathrm{dt}, J=2.0,0.9$ $\mathrm{Hz}, 1 \mathrm{H}), 4.23-4.20(\mathrm{~m}, 1 \mathrm{H}), 2.70(\mathrm{ddd}, J=9.1,5.9,2.7 \mathrm{~Hz}, 1 \mathrm{H}), 2.44(\mathrm{dt}, J=6.7,1.3 \mathrm{~Hz}, 1 \mathrm{H})$, 2.27-2.16 (m, 1H), 2.08-1.97 (m, 1H), 1.93-1.81 (m, 2H), 1.78-1.66 (m, 8H), 1.64-1.58 (m, 1H), $1.55-1.48(\mathrm{~m}, 1 \mathrm{H}), 0.90(\mathrm{~s}, 9 \mathrm{H}), 0.12(\mathrm{~s}, 3 \mathrm{H}), 0.11(\mathrm{~s}, 3 \mathrm{H}) ;{ }^{13} \mathrm{C} \mathrm{NMR}\left(126 \mathrm{MHz}, \mathrm{CDCl}_{3}\right) \delta 174.9$, $148.2,136.5,133.6,133.5,117.0,111.2,85.8,67.5,58.6,49.1,44.3,38.9,34.7,31.5,26.1,23.5$, 
22.4, 22.3, 20.5, 18.1,-3.6,-4.4; IR (Neat Film, NaCl) 2953, 2857, 1766, 1645, 1463, 1343, 1254, 1197, 1159, 1079, 1057, 1024, 891, 864, 833, 775, $673 \mathrm{~cm}^{-1}$; HRMS (MM+) m/z calc'd for $\mathrm{C}_{25} \mathrm{H}_{39} \mathrm{O}_{3} \mathrm{Si}[\mathrm{M}+\mathrm{H}]^{+}:$415.2663, found, 415.2697; $[\alpha]_{\mathrm{D}}^{25.0}-35.4^{\circ}\left(c 0.10, \mathrm{CHCl}_{3}\right)$.

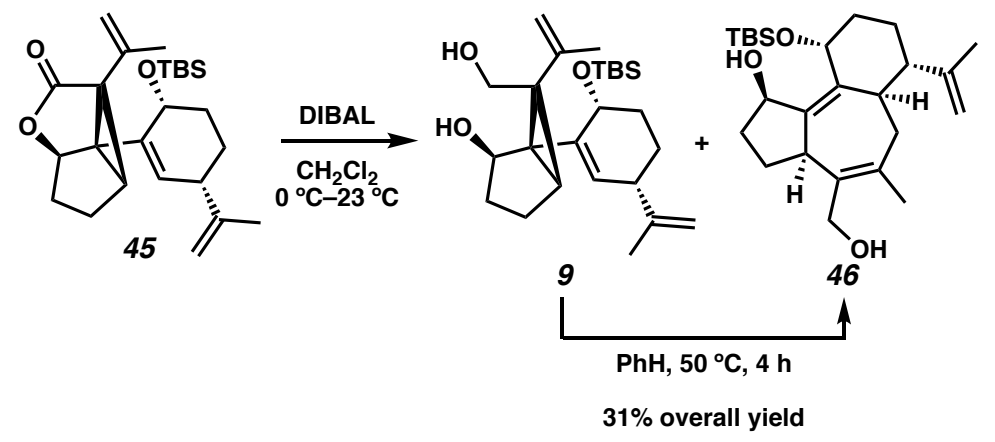

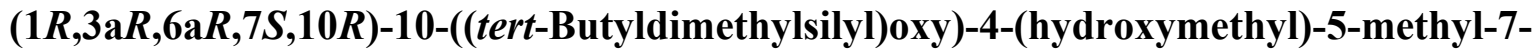

(prop-1-en-2-yl)-1,2,3,3a,6,6a,7,8,9,10-decahydrobenzo[e]azulen-1-ol (46): To a flame-dried round-bottom flask equipped with a magnetic stir bar were added vinyl lactone 45 (29 mg, 0.0699 mmol) and DCM (14 mL). The flask was cooled to $0{ }^{\circ} \mathrm{C}$ and stirred for $10 \mathrm{~min}$. A solution of DIBAL (1 M in DCM, $0.35 \mathrm{~mL}, 0.35 \mathrm{mmol}$ ) was added dropwise. The reaction mixture was slowly warmed up to $23{ }^{\circ} \mathrm{C}$ and remained to stir for $24 \mathrm{~h}$. The reaction was quenched by methanol $(0.35$ $\mathrm{mL})$. Saturated aqueous potassium sodium tartrate solution $(3 \mathrm{~mL})$ was added to the mixture. The phases were separated and the aqueous phases were extracted with DCM $(5 \times 10 \mathrm{~mL})$. The combined organic phases were dried over $\mathrm{MgSO}_{4}$, filtered, and transferred to round-bottom flask. The mixture was concentrated under reduced pressure and dissolved in benzene. The flask was immersed in a $50^{\circ} \mathrm{C}$ oil bath. After $4 \mathrm{~h}$ of stirring, the reaction was cooled to ambient temperature and concentrated under reduced pressure. The residue was purified by flash column chromatography (1:1 hexanes: EtOAc) to afford diol 46 as a white solid (9.0 mg, $0.215 \mathrm{mmol}$, $31 \%$ yield); $\mathrm{R}_{f}=0.08(3: 1$ hexanes: EtOAc $) ;{ }^{1} \mathrm{H}$ NMR $\left(600 \mathrm{MHz}, \mathrm{C}_{6} \mathrm{D}_{6}\right) 5.00(\mathrm{dd}, J=4.1,1.9 \mathrm{~Hz}$, 1H), $4.92-4.89(\mathrm{~m}, 1 \mathrm{H}), 4.87(\mathrm{~d}, J=2.2 \mathrm{~Hz}, 1 \mathrm{H}), 4.83(\mathrm{~d}, J=4.2 \mathrm{~Hz}, 1 \mathrm{H}), 4.16(\mathrm{~d}, J=11.3 \mathrm{~Hz}$, 
$1 \mathrm{H}), 3.91(\mathrm{~d}, J=11.3 \mathrm{~Hz}, 1 \mathrm{H}), 3.56-3.49(\mathrm{~m}, 1 \mathrm{H}), 3.06-3.00(\mathrm{~m}, 1 \mathrm{H}), 2.85(\mathrm{dd}, J=13.8,4.5 \mathrm{~Hz}$, $1 \mathrm{H}), 2.38(\mathrm{dtd}, J=13.7,11.8,6.1 \mathrm{~Hz}, 1 \mathrm{H}), 2.28-2.13(\mathrm{~m}, 2 \mathrm{H}), 2.04(\mathrm{dd}, J=14.7,11.4 \mathrm{~Hz}, 1 \mathrm{H})$, 1.92-1.84 (m, 2H), $1.81(\mathrm{~d}, J=1.7 \mathrm{~Hz}, 3 \mathrm{H}), 1.77(\mathrm{~d}, J=1.2 \mathrm{~Hz}, 3 \mathrm{H}), 1.76-1.70(\mathrm{~m}, 1 \mathrm{H}), 1.54$ $(\mathrm{tdd}, J=13.0,4.3,2.0 \mathrm{~Hz}, 1 \mathrm{H}), 1.51-1.37(\mathrm{~m}, 2 \mathrm{H}), 1.01(\mathrm{~s}, 9 \mathrm{H}), 0.08(\mathrm{~s}, 3 \mathrm{H}), 0.08(\mathrm{~s}, 3 \mathrm{H}) ; \delta^{13} \mathrm{C}$ NMR (101 MHz, DMSO-d6) 148.5, 140.1, 138.8, 137.8, 132.4, 111.9, 71. 3, 68.8, 57.9, 49.1, 42.1, 34.4, 34.0, 33.8, 29.3, 26.7, 26.6, 25.8, 25.7, 21.5, 17.7, -4.5, -4.7; IR (Neat Film, NaCl) 3342, 2929, 2856, 1645, 1451, 1254, 1163, 1079, 1033, 890, 836, 773, 739, $702 \mathrm{~cm}^{-1}$; HRMS (FAB+) $m / z$ calc'd for $\mathrm{C}_{25} \mathrm{H}_{41} \mathrm{O}_{3} \mathrm{Si}\left[\mathrm{M}+\mathrm{H}-\mathrm{H}_{2}\right]^{+}: 417.2825$, found $417.2833 ;[\alpha]_{\mathrm{D}}{ }^{25.0}-27.6^{\circ}$ (c 0.10 , $\left.\mathrm{CH}_{3} \mathrm{OH}\right)$. 


\section{References}

(1) Pangborn, A. B.; Giardello, M. A.; Grubbs, R. H.; Rosen, R. K.; Timmers, F. J., Safe and Convenient Procedure for Solvent Purification. Organometallics 1996, 15, 15181520.

(2) Davies, H. M. L.; Cantrell, R. W.; Jr.; Romines, R. K.; and Baum, S. J., Synthesis of Furans via Rhodium(II) Acetate-Catalyzed Reaction of Acetylenes with $\alpha$ Diazocarbonyls: Ethyl 2-Methyl-5-Phenyl-3-Furancarboxylate. Org. Synth. 1992, 70, 93100; Coll. Vol. IX 1998, 422-426.

(3) Charles, R. G., Copper (II) and Nickel (II) N-(n-alkyl)salycyladmine Chelates. $J$. Org. Chem. 1957, 22, 677-679.

(4) McUliffe, C. A.; Hosseiny, A.; McCullough, F. P., The chemistry of molybdenum and tungsten. Part XIV. Oxomolybdenum(V) complexes of quinolines. Inorg. Chim. Acta 1979, 33, 5-10.

(5) Wang, Q.; Fan, S. Y.; Wong, H. N. C.; Li, Z.; Fung, B. M.; Twieg, R. J.; Nguyen, H. T., Enantioselective Synthesis of Chiral Liquid Crystalline Compounds from Monoterpenes. Tetrahedron 1993, 49, 619-638.

(6) Seigel, C.; Gordon, P. M.; Razdan, R. K., An Optically Active Terpenic Synthon for $\Delta$ 9-Cannabinoids: Synthesis of (-)-11-Hydroxy- $\Delta$ 9-tetrahydrocannabinol (THC) and its 1',1'-Dimethylheptyl Analog. J. Org. Chem. 1989, 54, 5428-5430. 


\section{NMR and IR Spectra of Unknown Compounds}

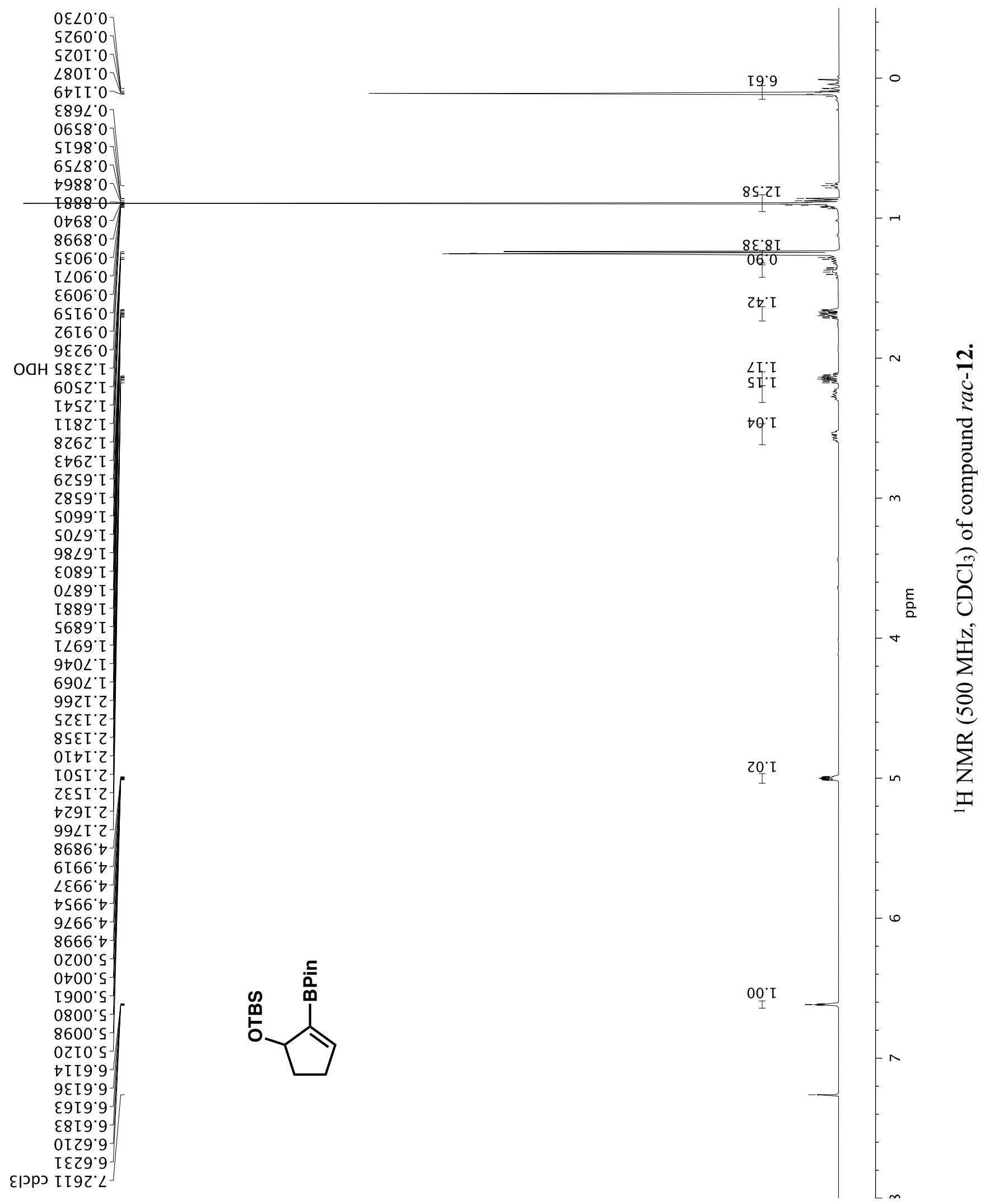




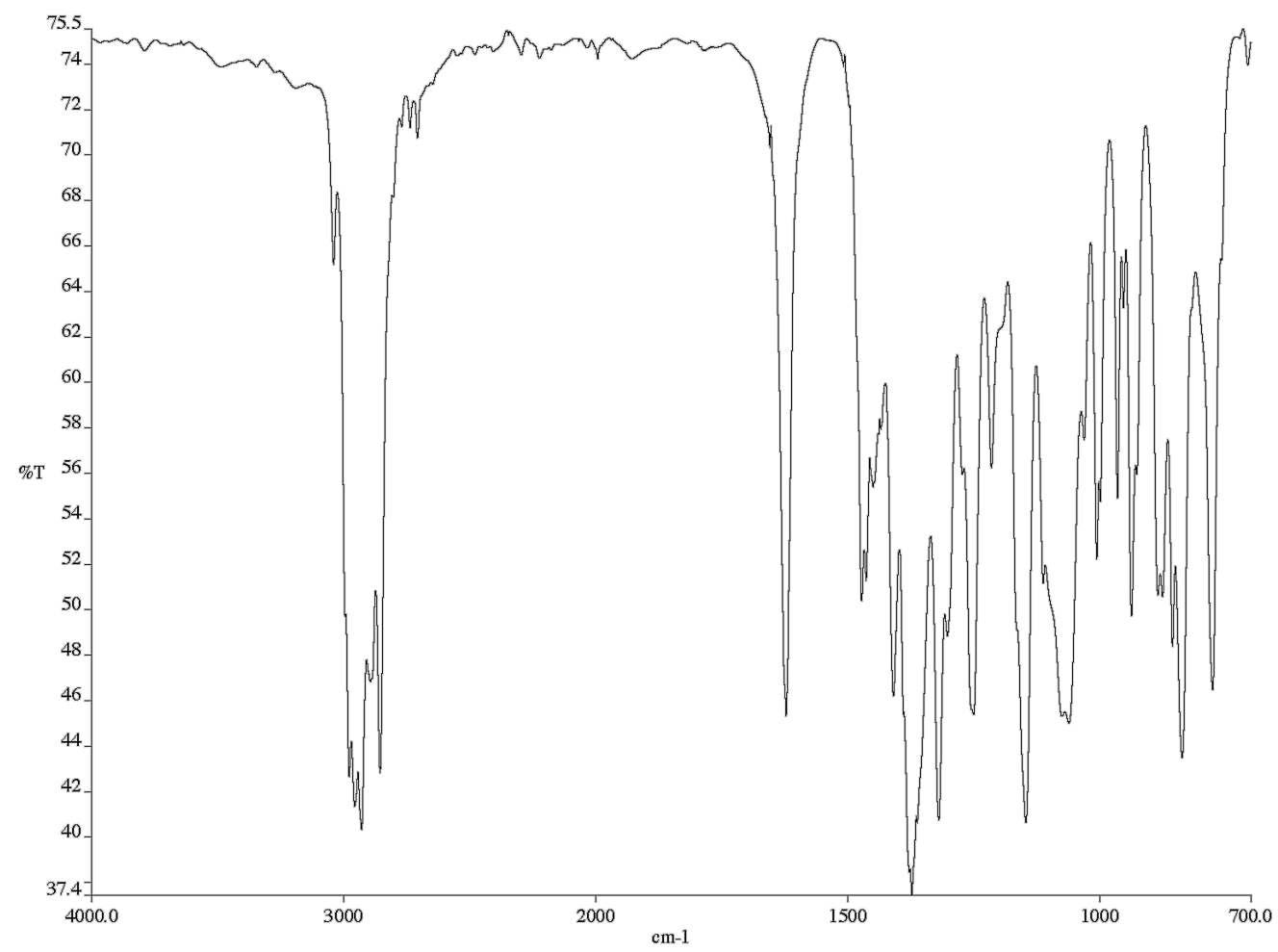

Infrared spectrum (Thin Film, NaCl) of compound rac-12.

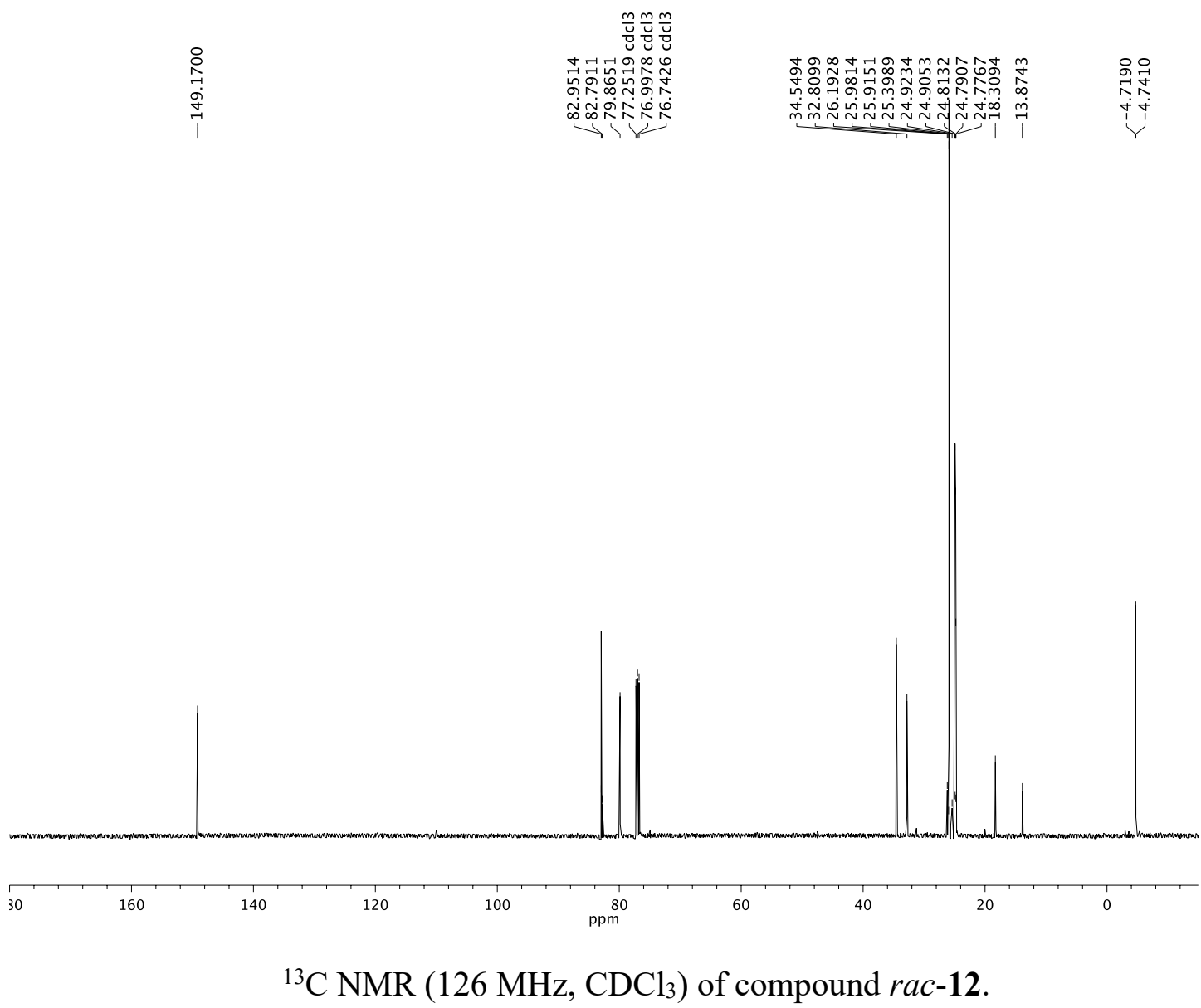




$$
\begin{aligned}
& \text { ZSSS I - } \\
& \text { 28S5. I } \\
& \angle \angle 9 S^{\circ} \mathrm{T} \\
& 6 \angle \angle S^{\circ} \mathrm{I} \\
& 608 \mathrm{~S}^{\circ} \mathrm{I} \\
& \text { \& } 6 S^{\circ} \text {. } \\
& \downarrow t 65^{\circ} \text {. } \\
& \text { 6209. I } \\
& 2909^{\circ} \mathrm{I} \\
& \text { SOL9. I } \\
& \text { S9I 9. I } \\
& \angle 8 \text { I }^{\circ} \mathrm{L} \\
& \text { ¿629. [ } \\
& \text { 6ع99. I - }
\end{aligned}
$$

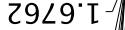

$$
\begin{aligned}
& \text { 2989. I - } \\
& 8889^{\circ} \mathrm{L} \\
& \text { 6869. I } \\
& \text { ZI0L' I } \\
& \text { ZEOL' I } \\
& 0 \angle \forall 8^{\circ} \mathrm{I} \\
& 0858^{\circ} \mathrm{I}- \\
& \angle 298^{\circ} \mathrm{I} \\
& \text { S } \angle 98^{\circ} \mathrm{I} \\
& 8698^{\circ} \mathrm{T} \\
& 8 \rightarrow \angle 8^{\circ} \mathrm{I}- \\
& \varepsilon 6 \angle 8^{\circ} \mathrm{I}- \\
& 6588^{\circ} \mathrm{L} \text { - } \\
& 9068^{\circ} \mathrm{I}- \\
& \text { ZS68 } \mathrm{L}- \\
& \text { L6E I' } 2 \\
& 9 \forall \succ\left[{ }^{\circ} 乙-\right. \\
& \varepsilon \angle \nabla I^{\circ} Z- \\
& \angle O S I^{\circ} Z \\
& \text { 乙†9 ' } 2 \text { - } \\
& \angle 89 \text { I }^{\circ} \mathrm{C} \\
& 8 \varepsilon \angle L^{\circ} Z- \\
& \text { T } 8 \angle L^{\circ} Z \\
& \text { દZ8I' } 2 \\
& 898 \text { I }^{\prime} \mathrm{C} \\
& 8 \mathrm{I}^{\circ} \mathrm{I}^{\prime} \mathrm{Z} \\
& \text { 096 I' } 2 \\
& \text { 266 ' } 2 \\
& \angle 00 Z^{\prime} Z \\
& \text { SSOZ'Z } \\
& 8602^{\prime} 2 \\
& \text { StI } Z^{\circ} Z \\
& 6 \angle I Z \cdot Z \\
& 8022^{\circ} 2 \\
& \text { ऽह乙2 Z }
\end{aligned}
$$

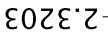

$$
\begin{aligned}
& \text { 9२乙ع 乙- } \\
& \text { ऽ200's } \\
& \downarrow 900 \text { 's } \\
& \text { E0I0 S } \\
& \text { 69I0's } \\
& \text { 9020 } \mathrm{S} \\
& \text { ह†२०० } \mathrm{s} \\
& \text { \& I6L's } \\
& \text { 0โ00.9 } \\
& 6900^{\circ} 9 \\
& \text { LI } 10.9 \text { - } \\
& \text { S } \angle\left[00^{\circ}\right. \text { ] } \\
& \text { عрро } 66 \mathrm{SZ}^{\circ} \mathrm{L}
\end{aligned}
$$

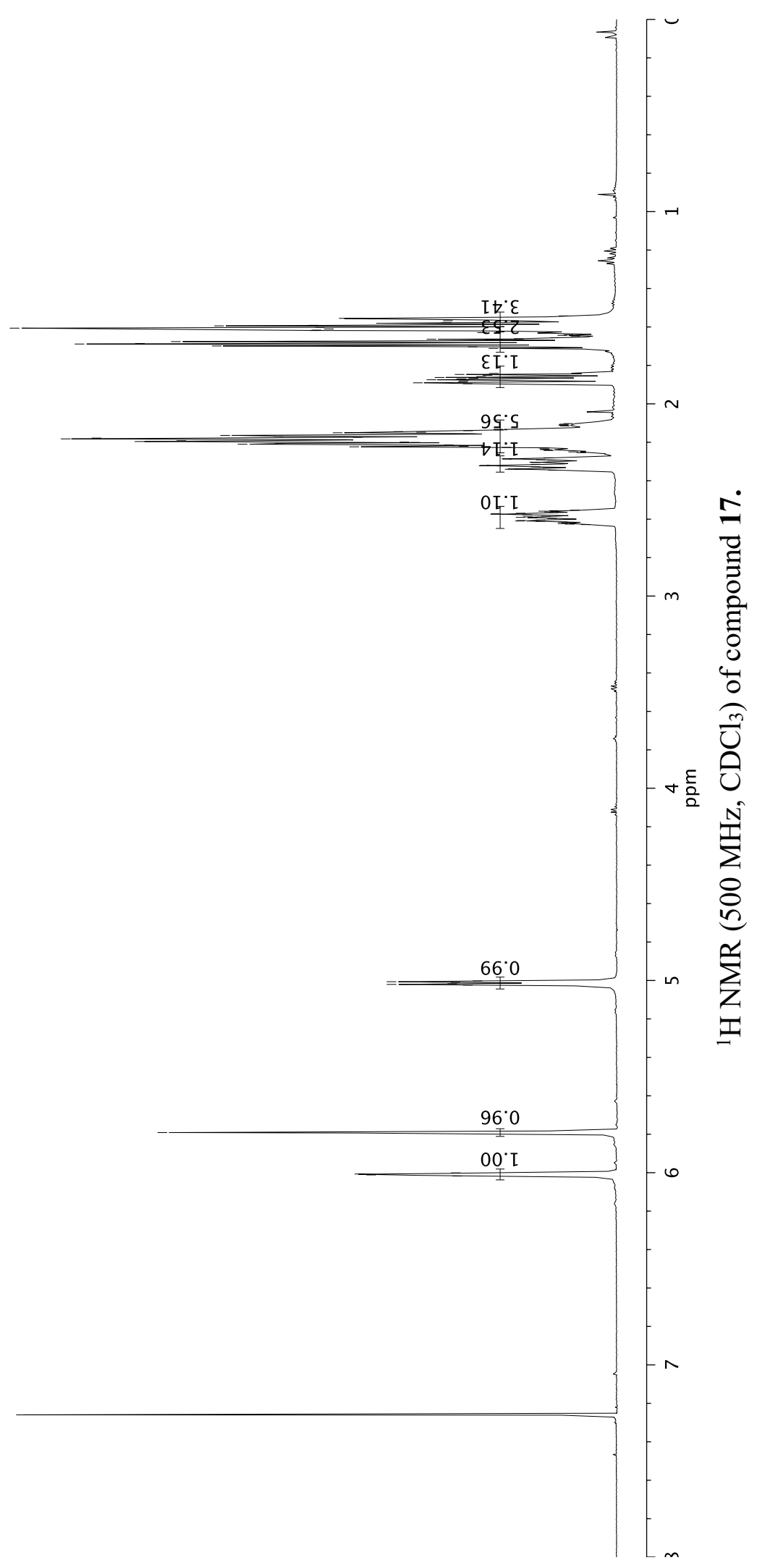




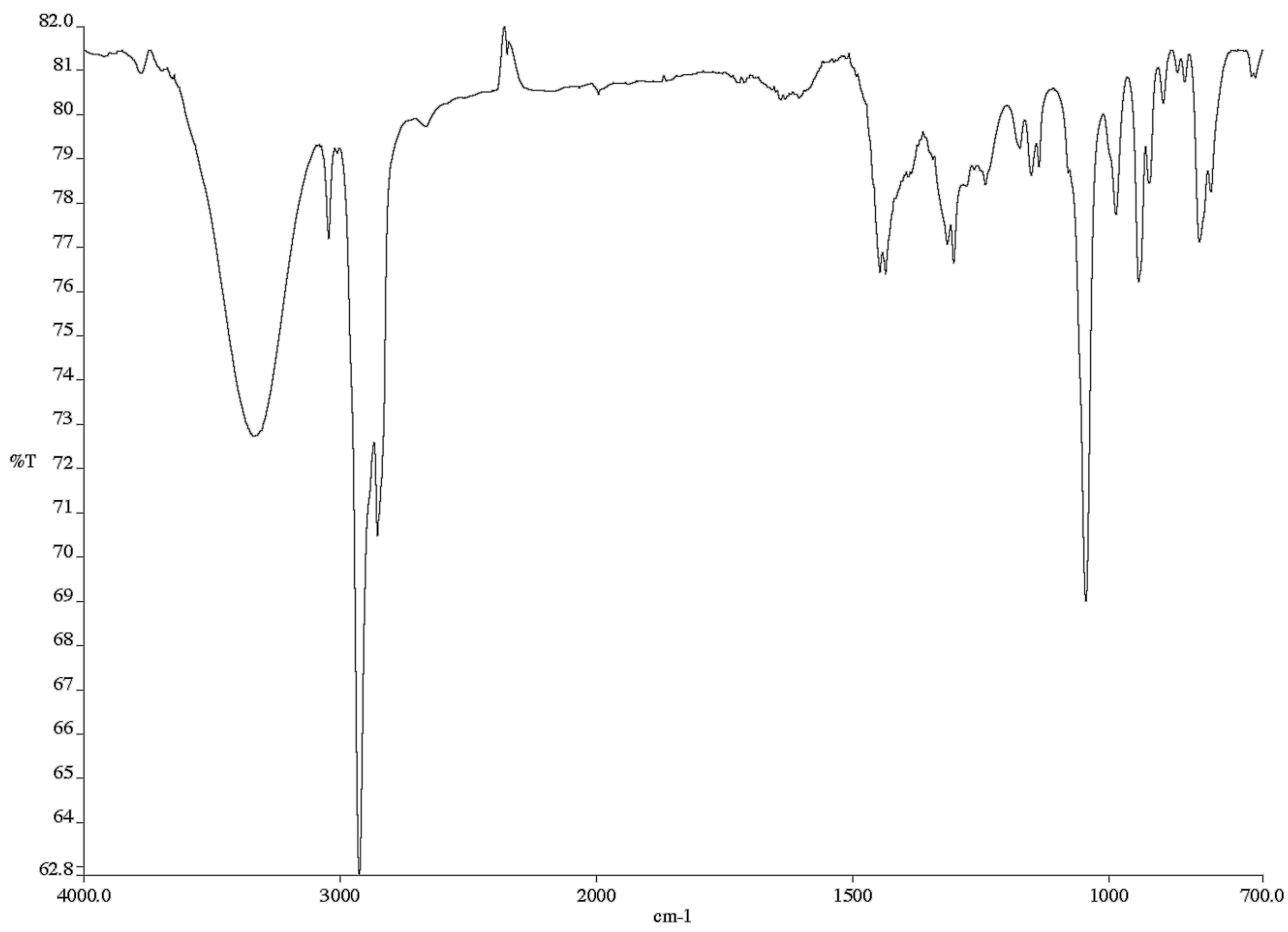

Infrared spectrum (Thin Film, $\mathrm{NaCl}$ ) of compound 17.
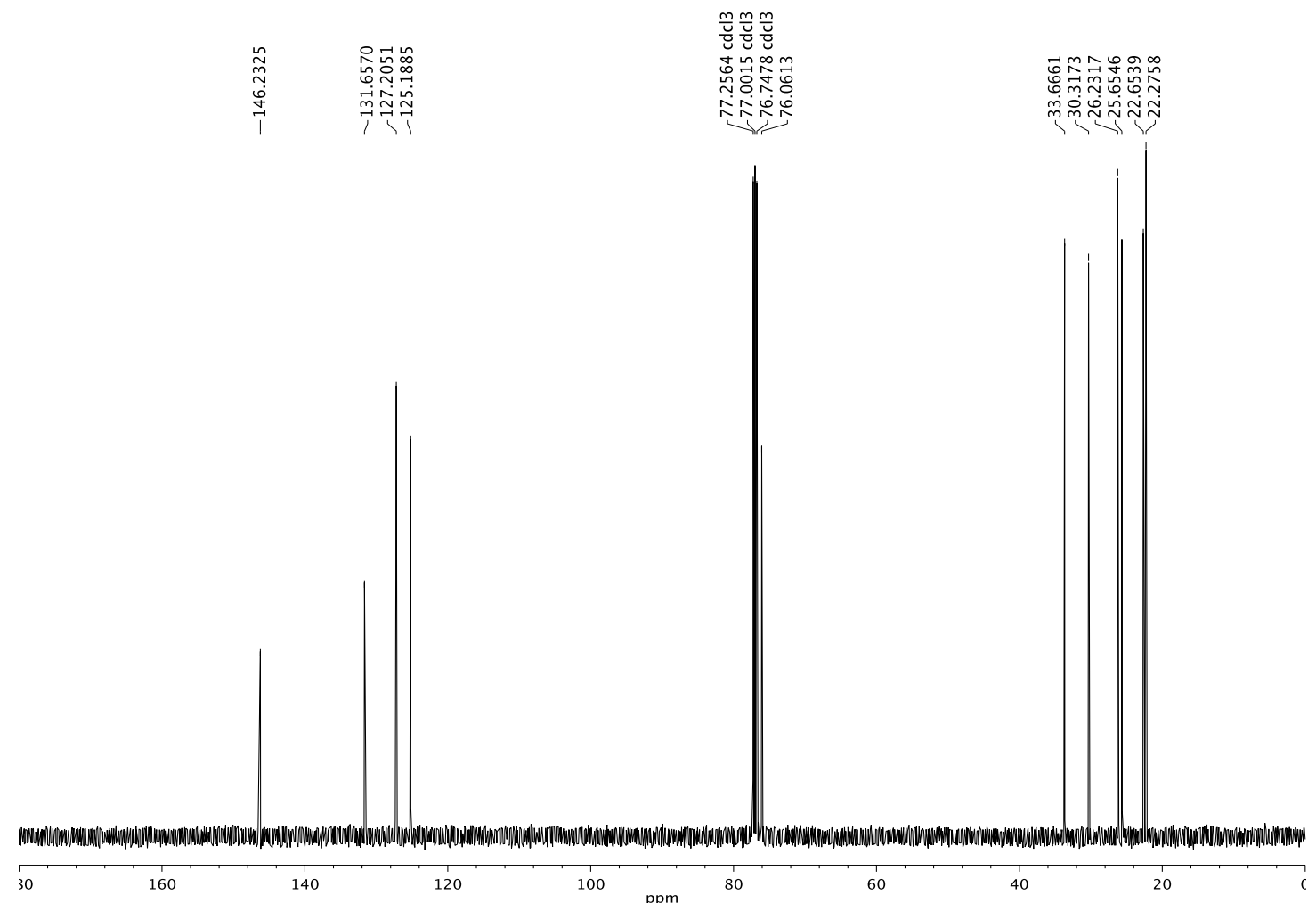

${ }^{13} \mathrm{C}$ NMR (126 MHz, $\mathrm{CDCl}_{3}$ ) of compound 17. 


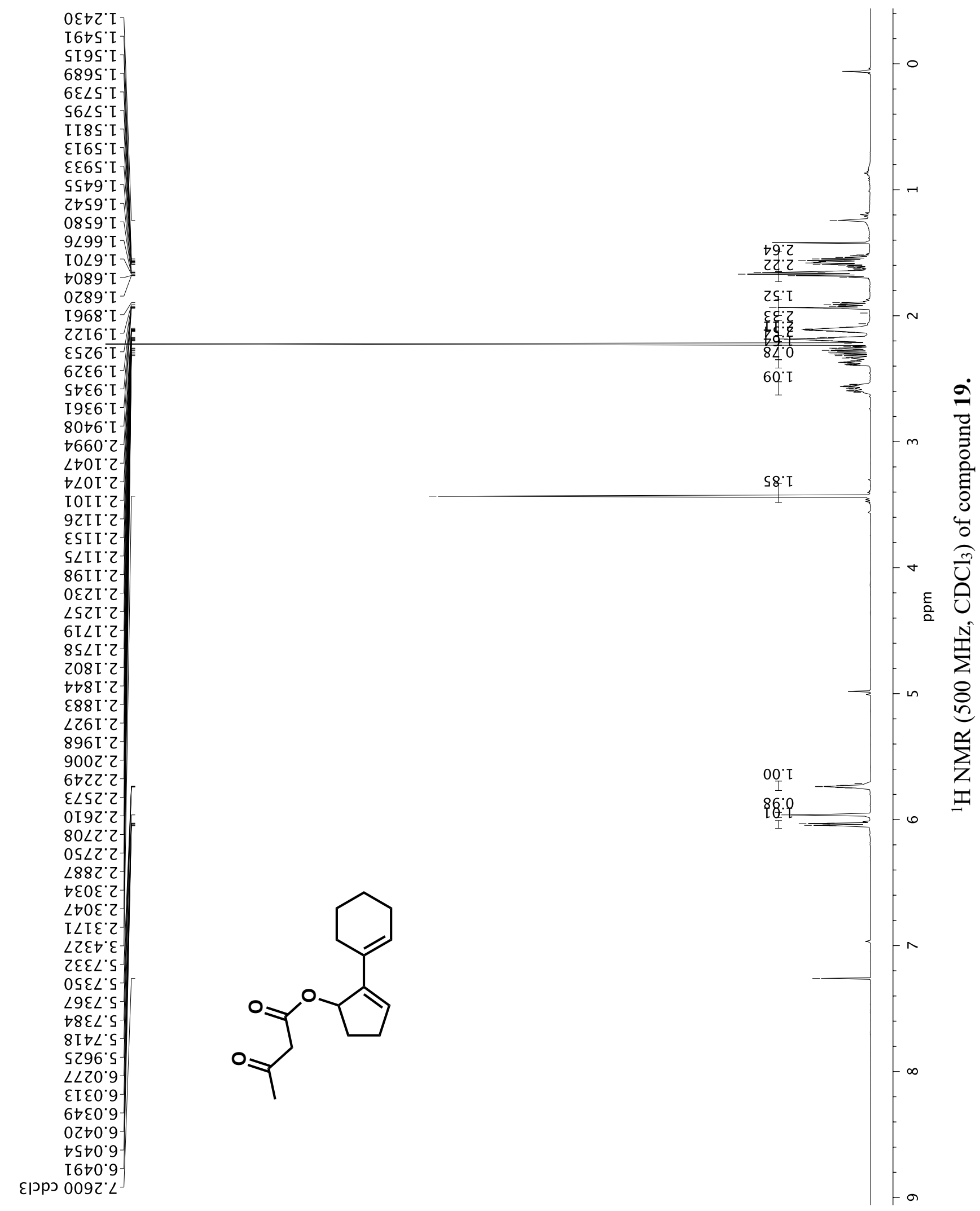




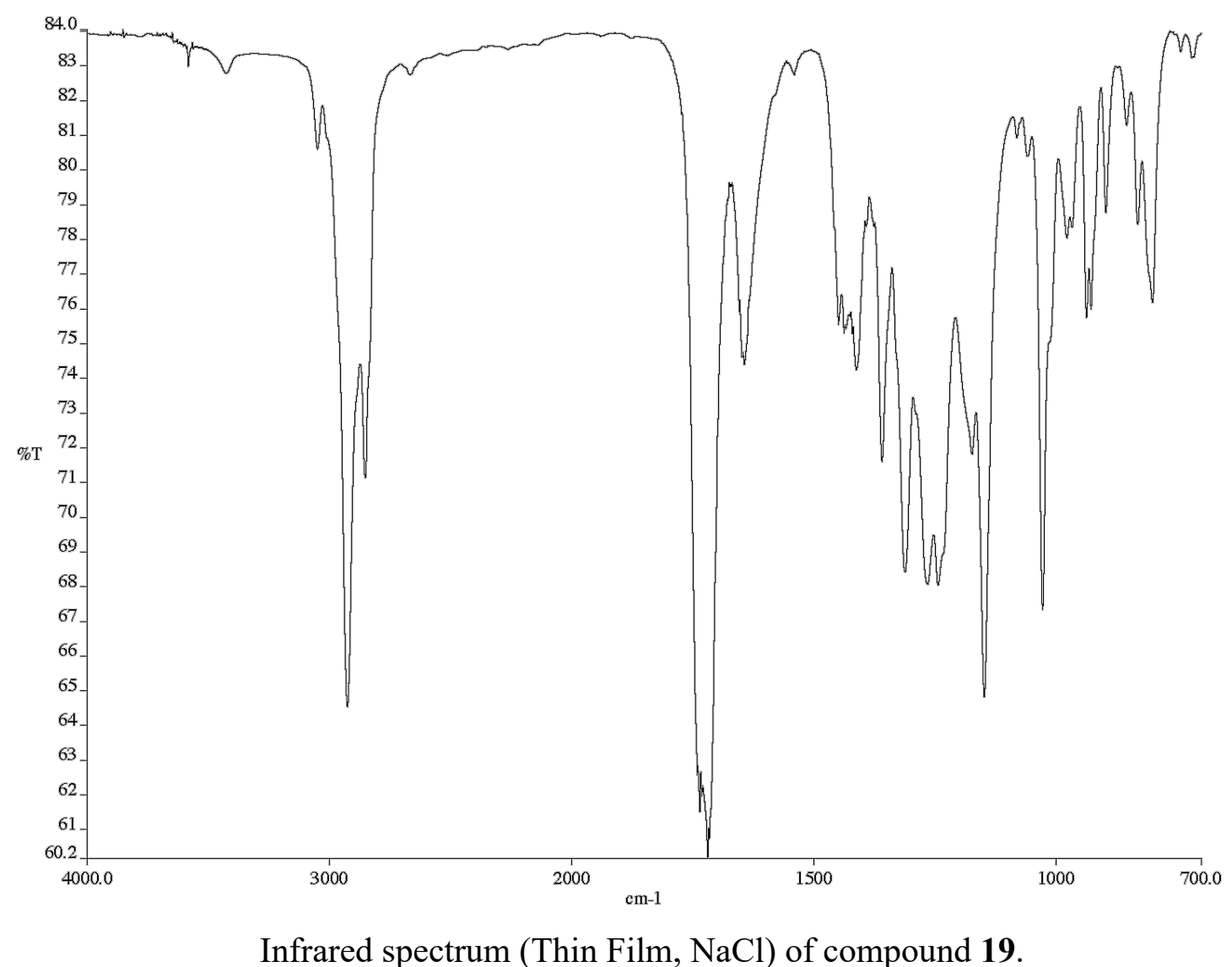

Infrared spectrum (Thin Film, NaCl) of compound 19.
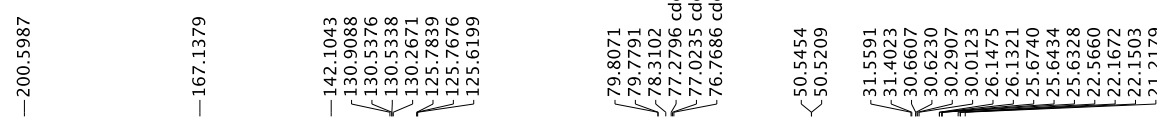

ํํำ

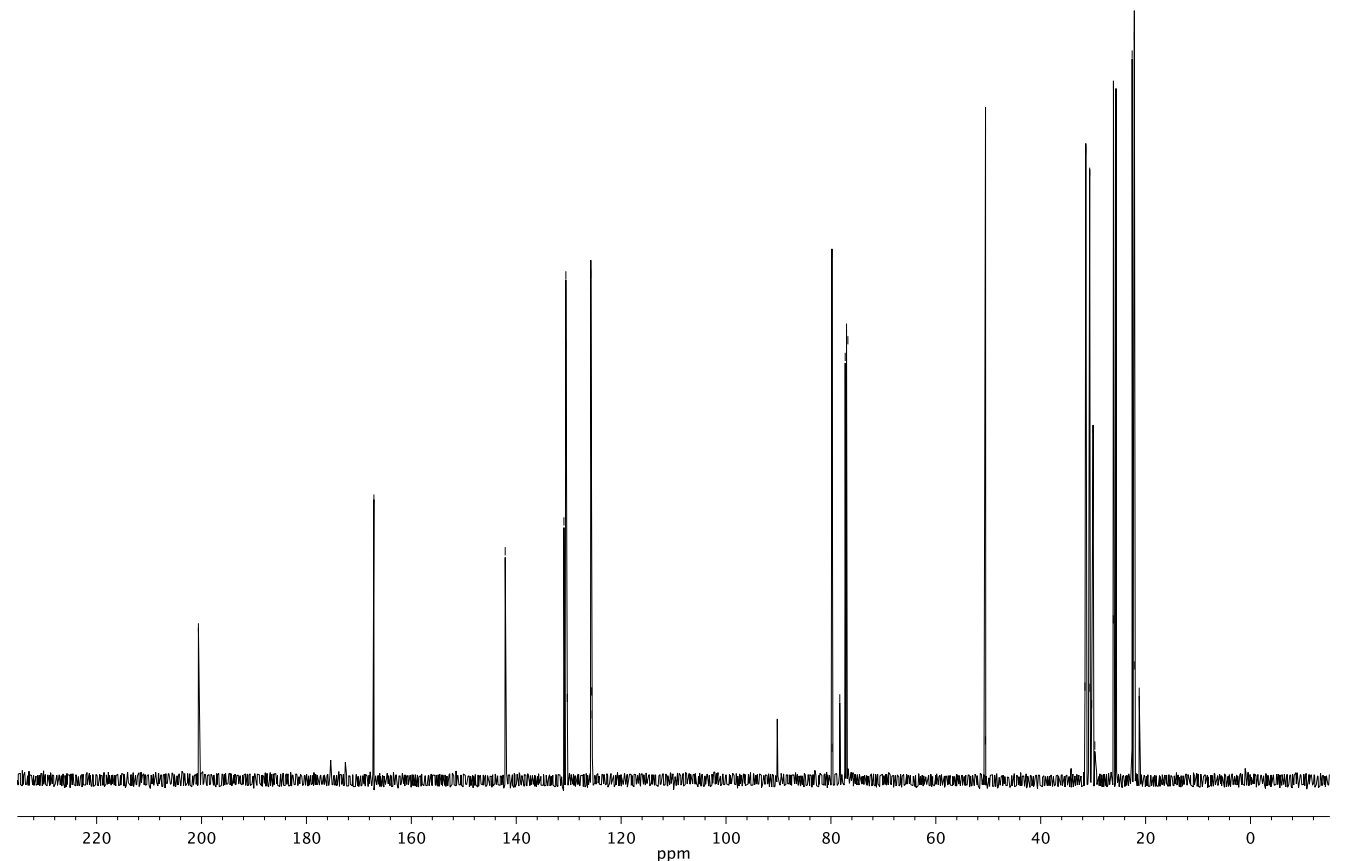

${ }^{13} \mathrm{C}$ NMR $\left(126 \mathrm{MHz}, \mathrm{CDCl}_{3}\right)$ of compound 19. 


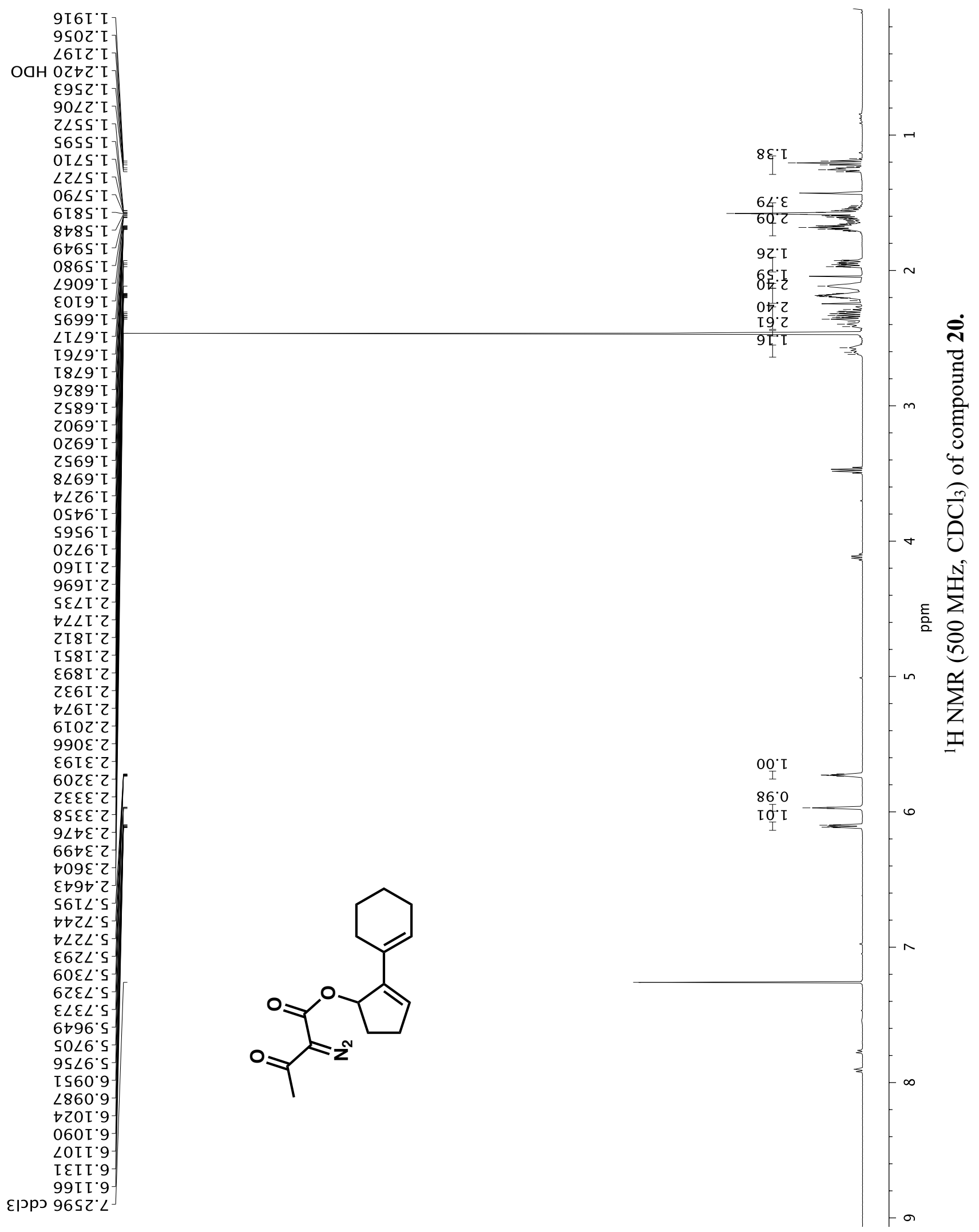



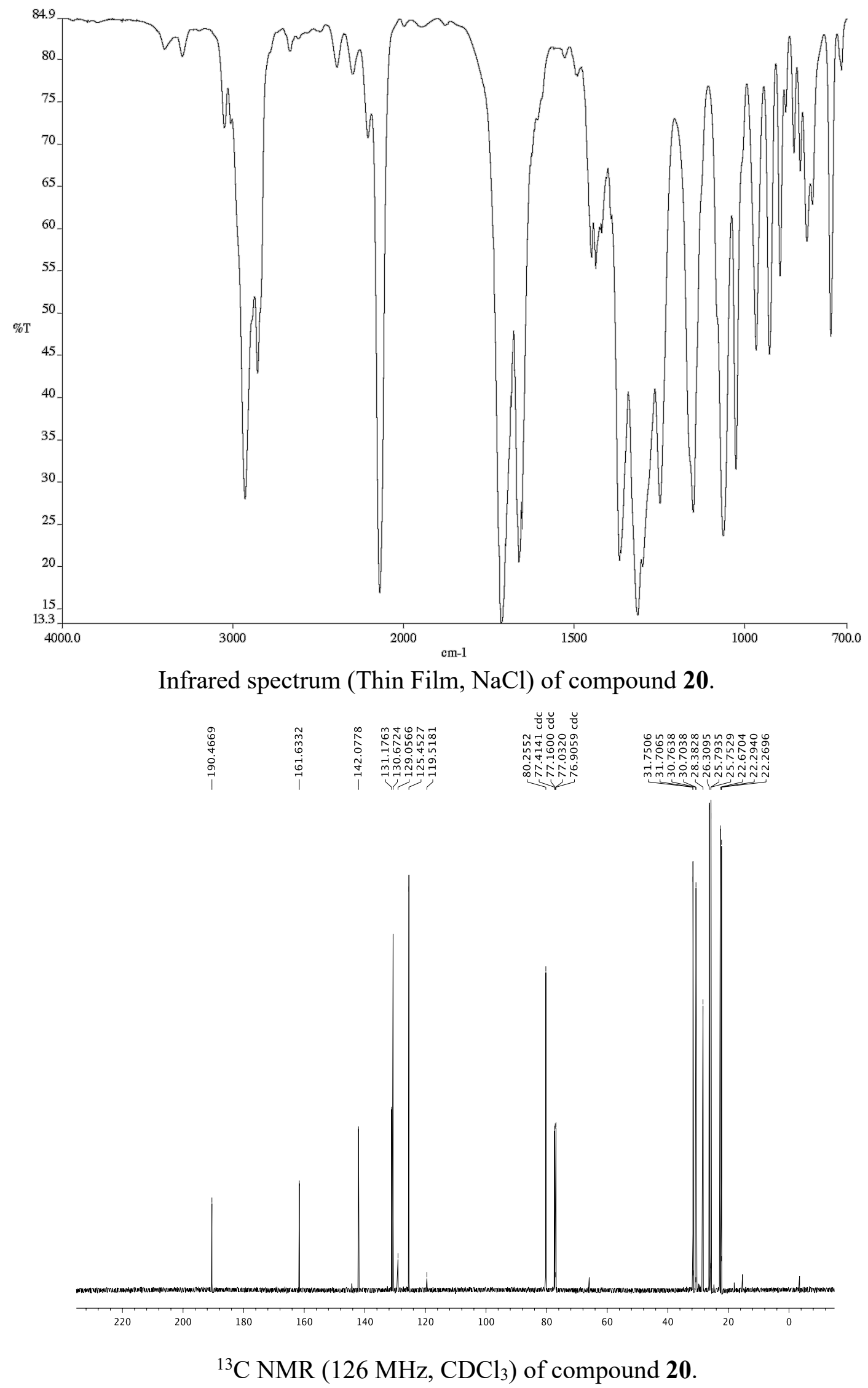
$\downarrow 9 \angle 8^{\circ} 0$

$6 S^{\circ} S^{\circ}[$

$\checkmark \angle Z S^{\circ} \mathrm{L}$

$88 \& S^{\circ} \mathrm{L}-$

$08+S^{\circ} \cdot$

† SS '

$86 \mathrm{SS}^{\circ} \mathrm{T}$

$08 \angle S^{\circ} T_{-}$

[88 $8 \mathrm{~S}^{\circ} \mathrm{L}$

S06S' I -

¿S6 $S^{\circ} \mathrm{L}$

$\downarrow 96 S^{\circ} \mathrm{L}$

966 '. I

6909. I

9809. I

82 โ9. I

$\angle 919^{\circ} \mathrm{T}$

SIZ9. I

$\angle \varepsilon Z 9^{\circ} \mathrm{I}$

$8 \angle 29^{\circ} \mathrm{I}$

¿629. I-

$\mathrm{S} 68 \mathrm{~L}^{\prime} \mathrm{I}-$

$\mathrm{Z6 \angle 8^{ \circ } \mathrm { I } -}$

2I $88^{\circ} \mathrm{T}$

S988. I

$0006^{\circ} \mathrm{I}$

$\angle \mathrm{LO6} \mathrm{6}^{\circ} \mathrm{I}$

$\neg S 06^{\circ} \mathrm{I}$

乙E66. I

$\nabla t 66^{\circ} \mathrm{I}$

$6 \mathrm{~S} 66^{\circ} \mathrm{T}$

$8 \triangleright 00^{\circ} \mathrm{Z}$

$7900^{\circ} z$

$0800^{\circ} \mathrm{Z}$

$\angle 8 \mathrm{LO}^{\circ} \mathrm{Z}$

$7020^{\circ} \mathrm{Z}$

2220.2

8520.2

90દ0 $Z$

† $\varepsilon 0^{\circ} 乙$

$\varepsilon \downarrow \varepsilon 0^{\circ} 乙$

$\varepsilon \angle \varepsilon 0^{\circ} Z$

86ध0.

$8 \forall t 0^{\circ} \mathrm{Z}$

$68+0.2$

2050.

† $250^{\circ} Z$

ऽะऽ० Z

L608' 2

$\varepsilon+9 t^{\circ} 2$

060 ' $\varepsilon$

$\downarrow$ I I I ' $\varepsilon$

SEI I ${ }^{\circ} \varepsilon$

$6 \mathrm{LZT} \varepsilon$

$\mathrm{I} \succ \mathrm{LI} \cdot \mathrm{C}$

$\downarrow 92 L^{\circ} \varepsilon$

S8 $28^{\circ} \circ$

I028 $\circ$

9I $28^{\circ} \circ$

$9 \varepsilon 28^{\circ} t$

$\angle S 28^{\circ} \circ$

$2 \angle 28^{\circ} \circ$

$\varepsilon \angle I \angle ' S$

SIZL'S

OSZL'S

$\angle 8 Z \angle ' S$

$\angle Z E L ' S$

عрगр $\triangleright 092^{\circ} L$

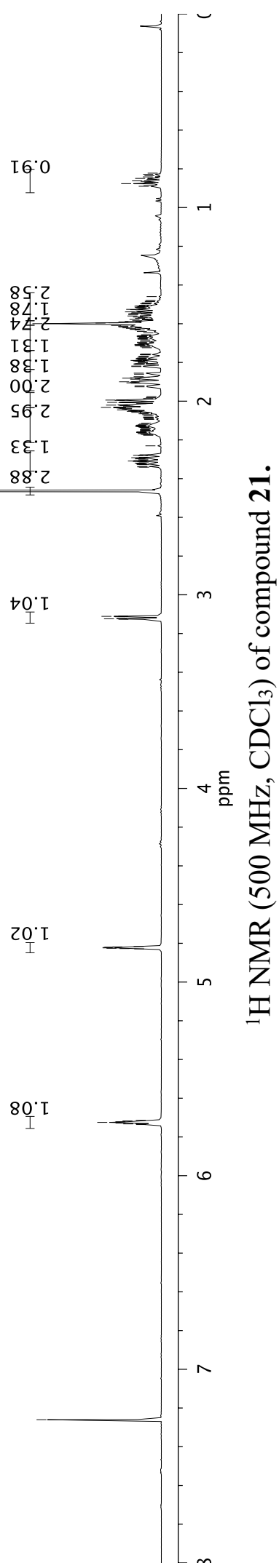



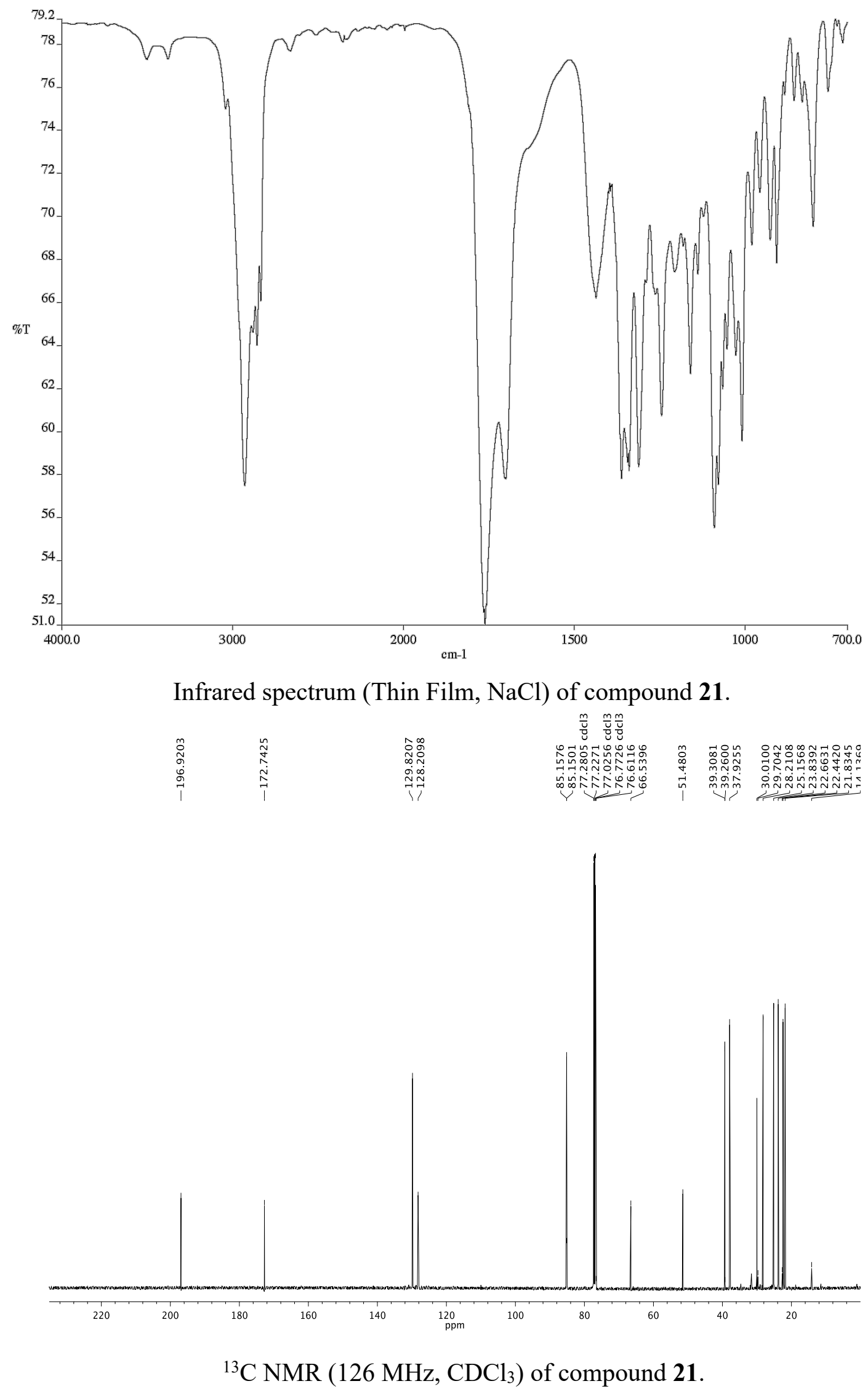


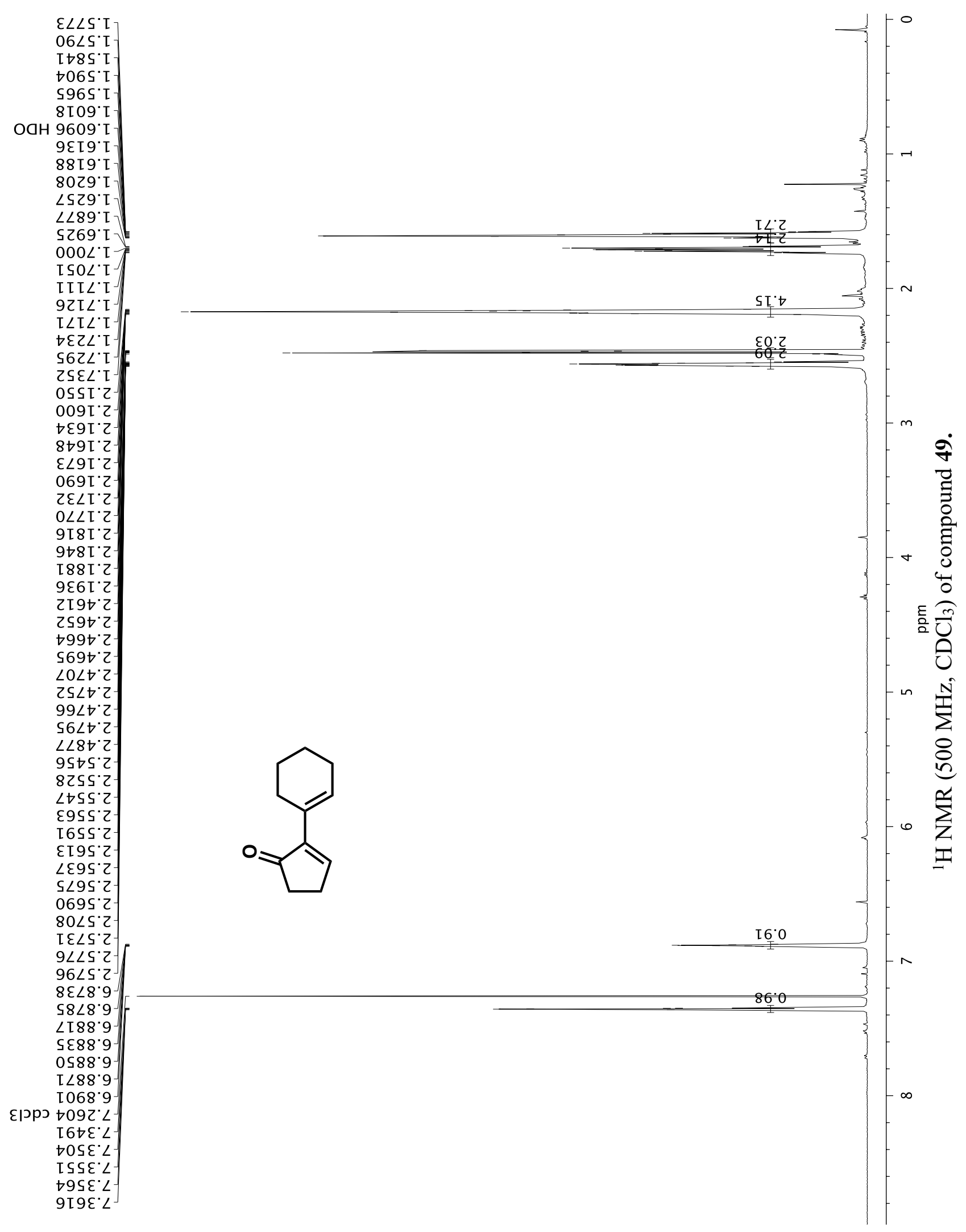



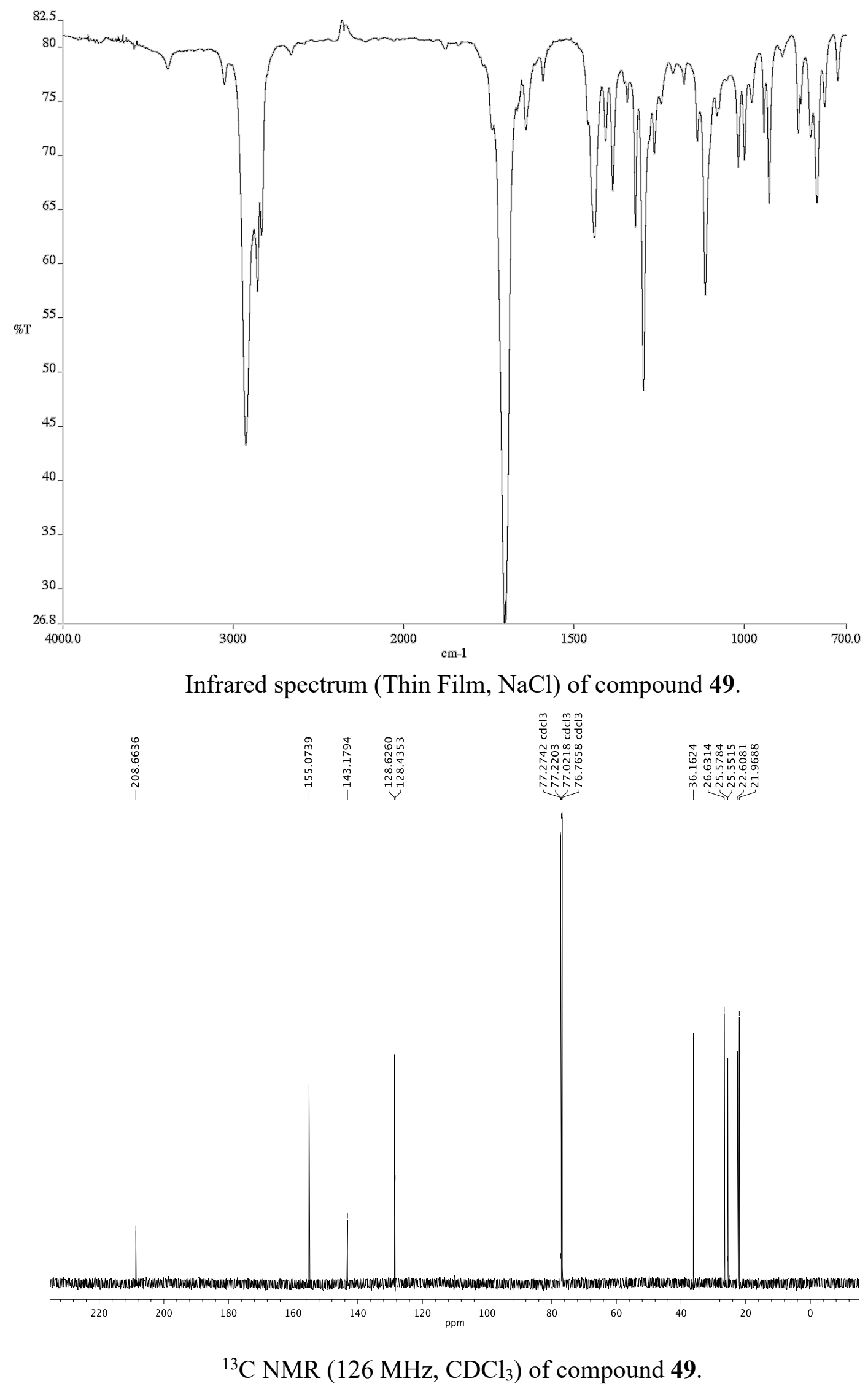


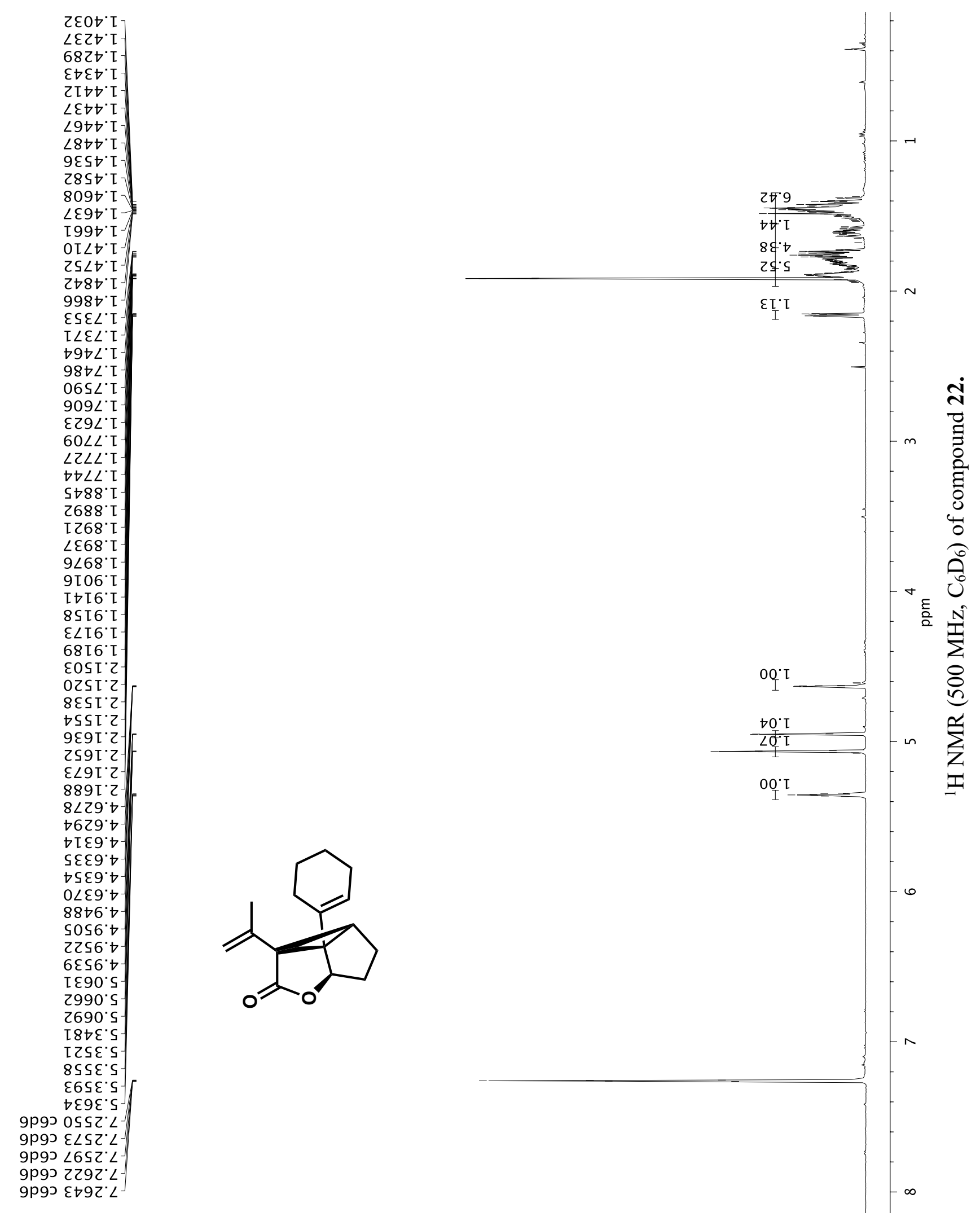




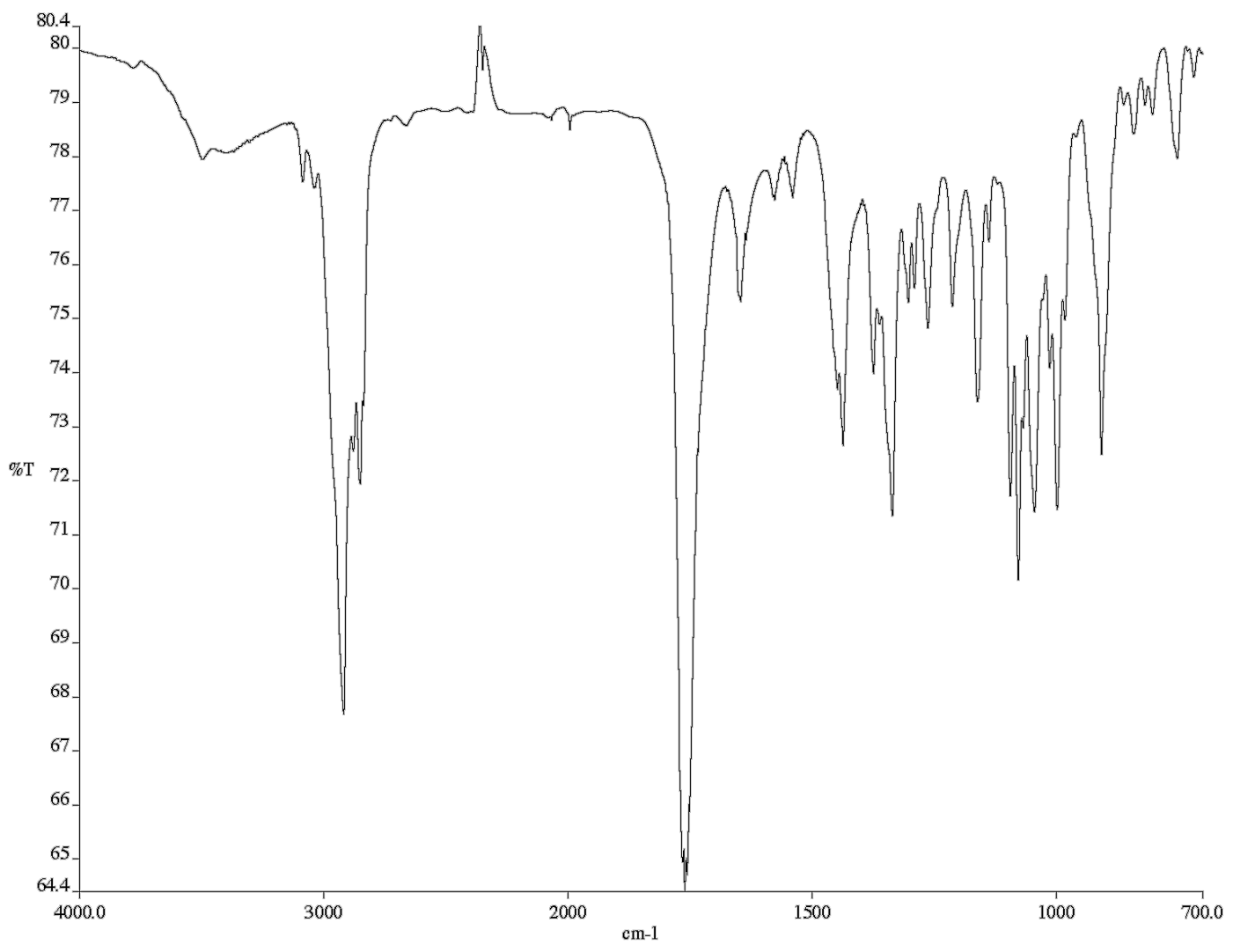

Infrared spectrum (Thin Film, $\mathrm{NaCl}$ ) of compound 22.

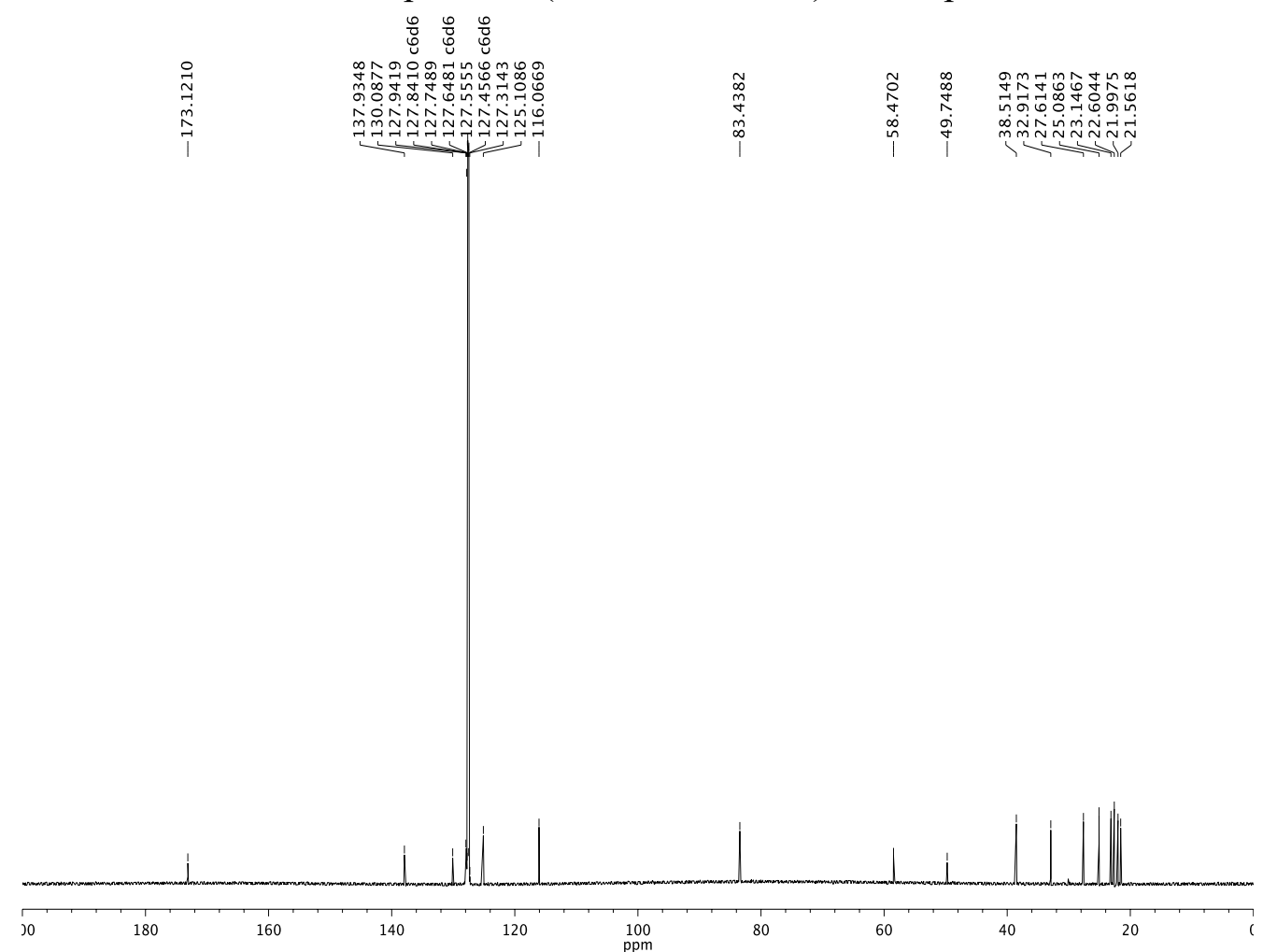

${ }^{13} \mathrm{C}$ NMR (126 MHz, $\left.\mathrm{C}_{6} \mathrm{Cl}_{6}\right)$ of compound 22. 

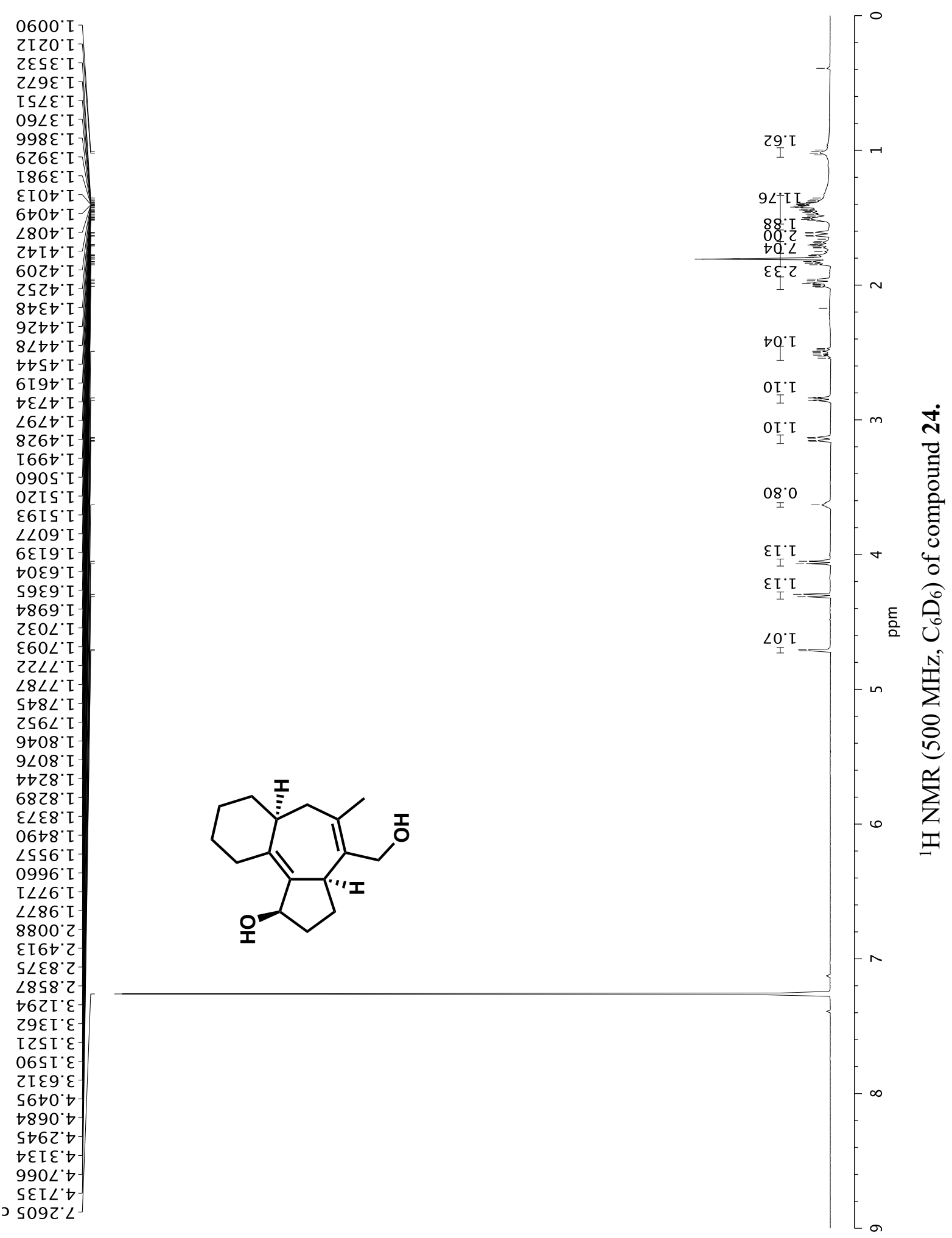

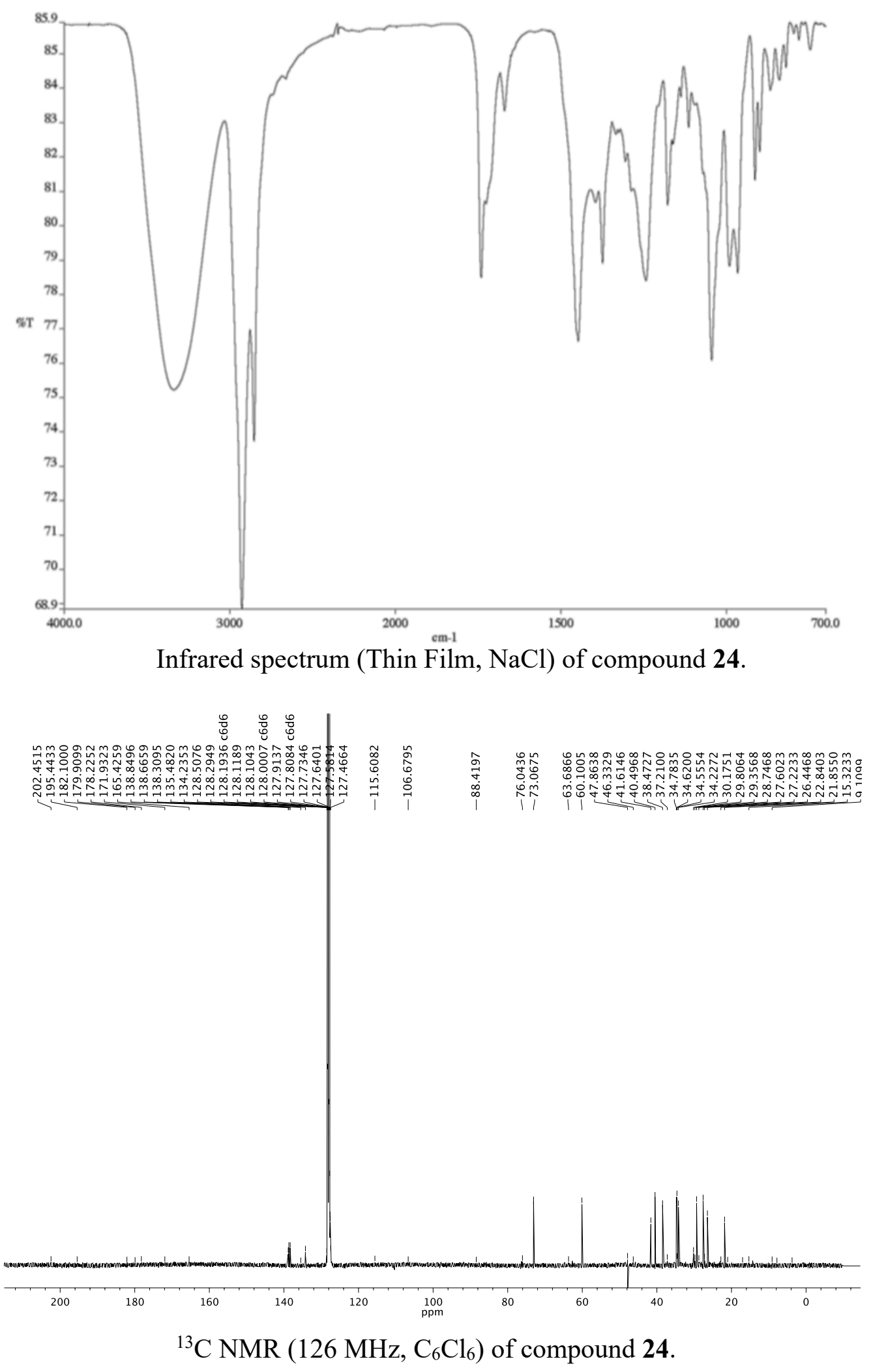


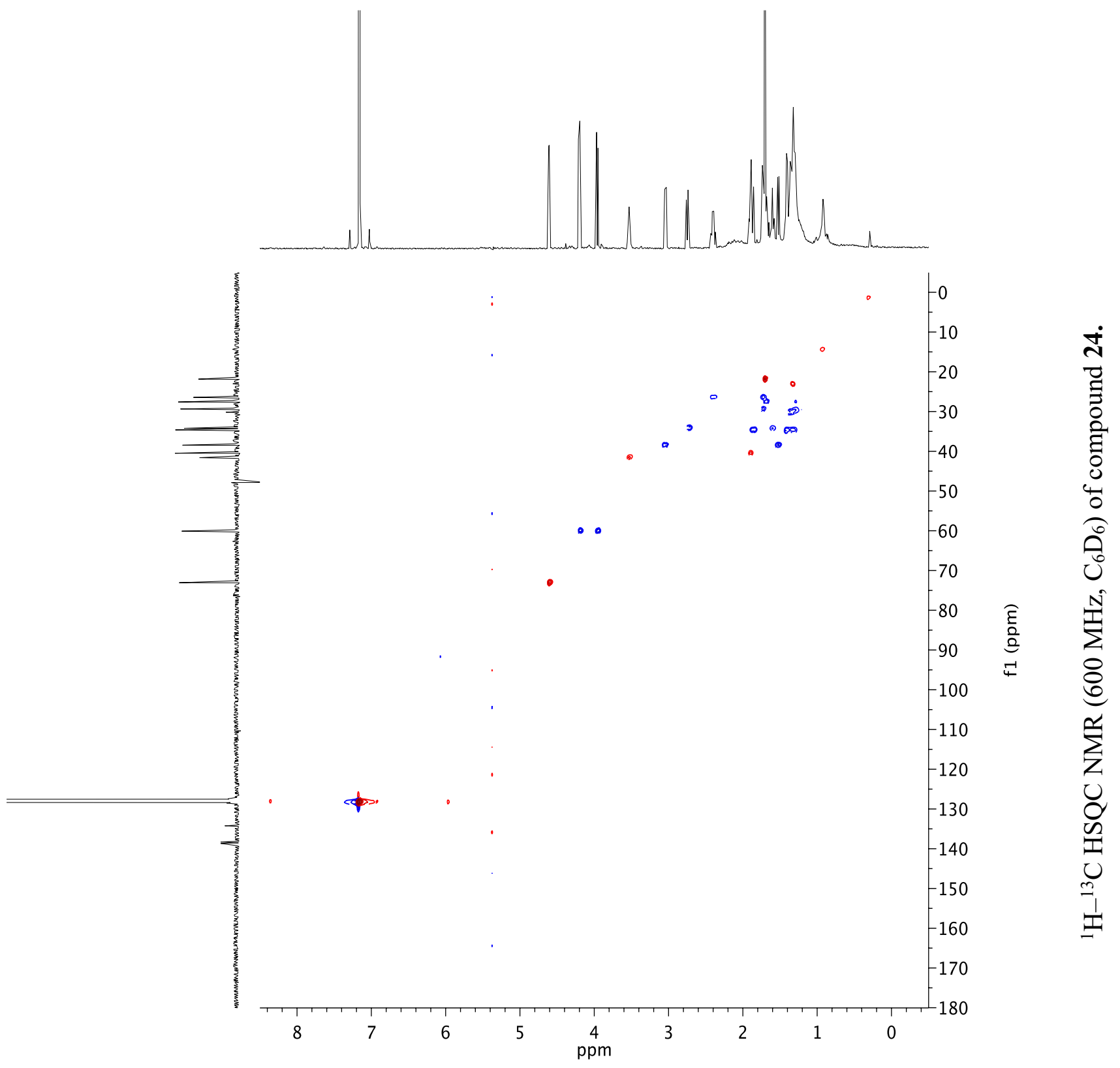




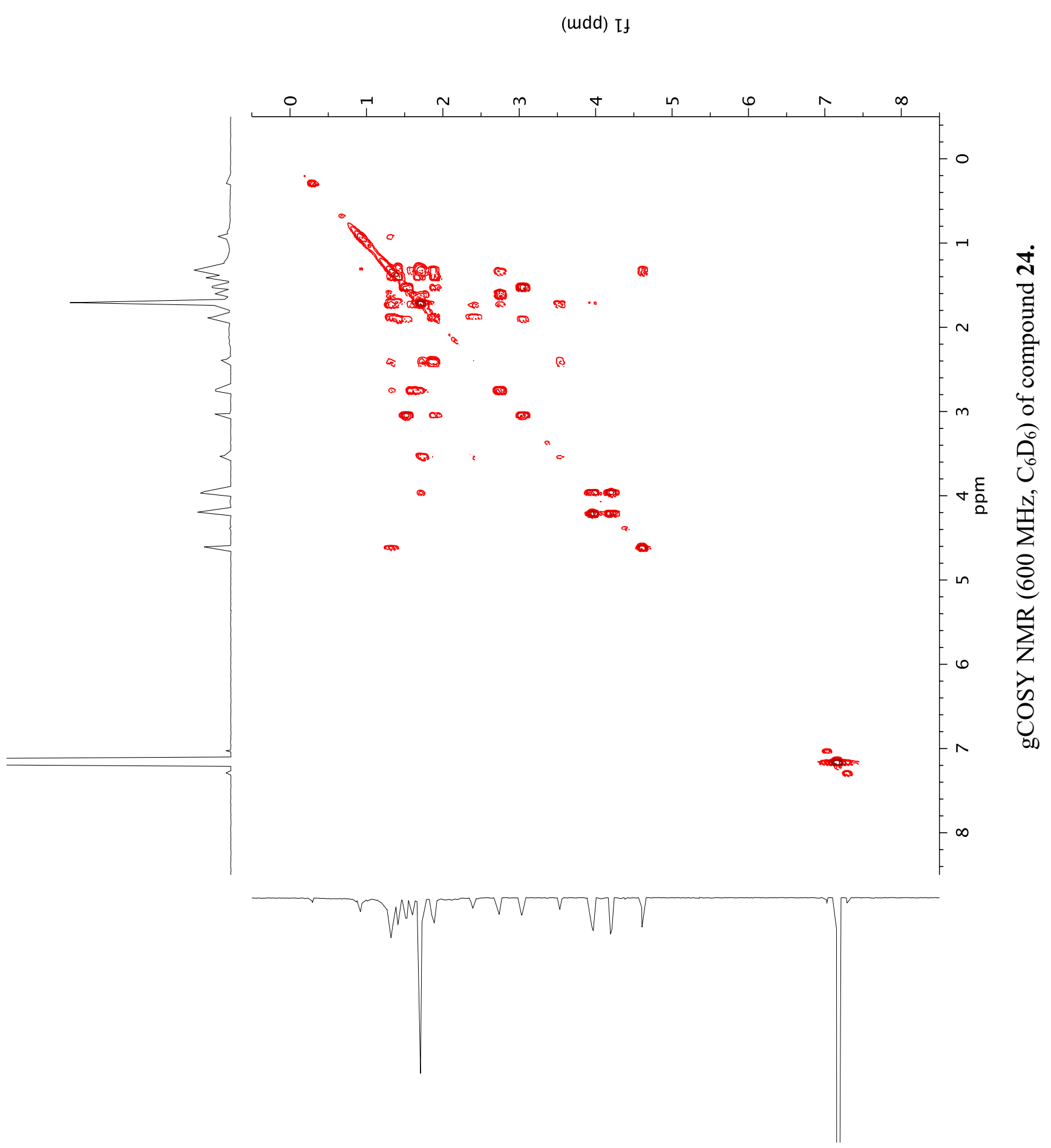




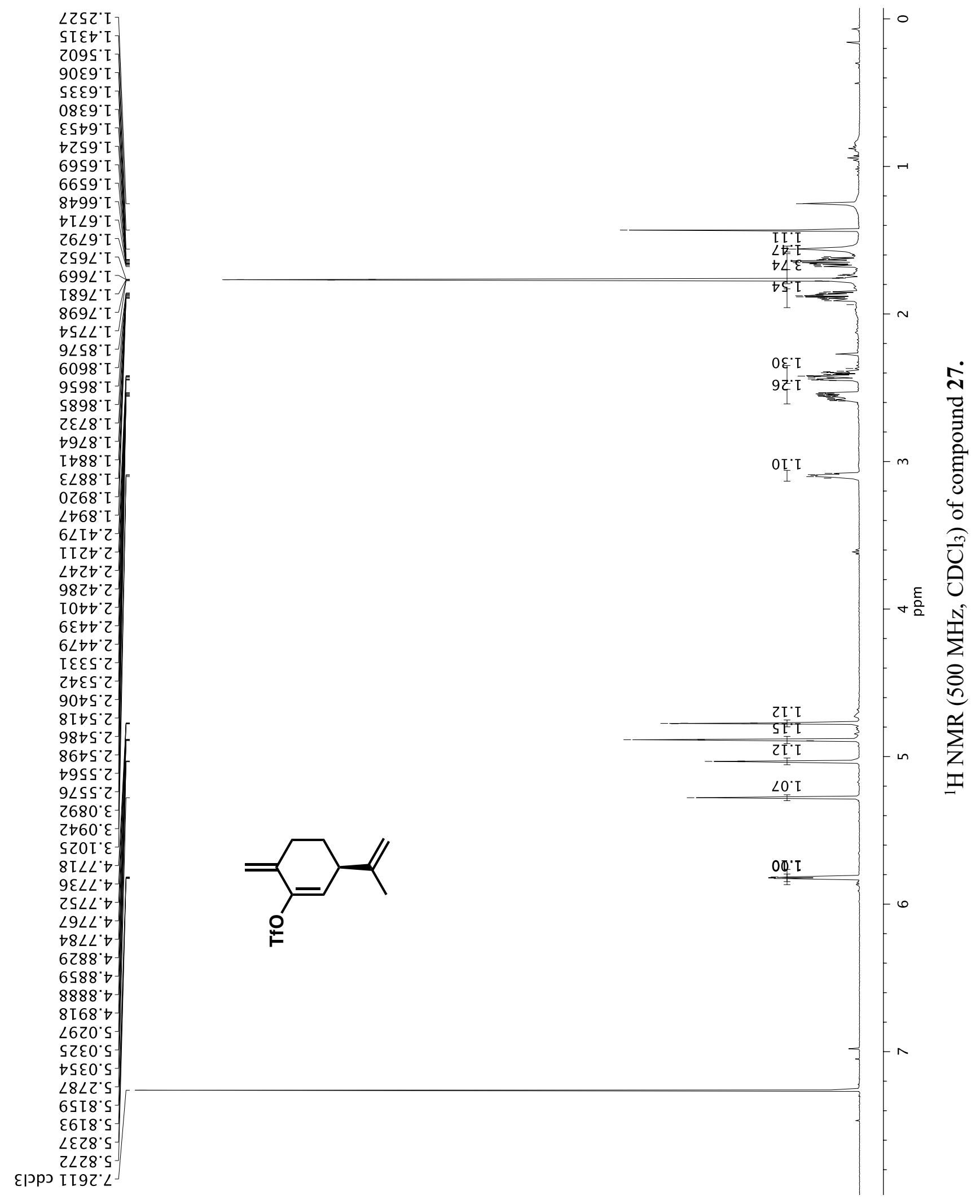




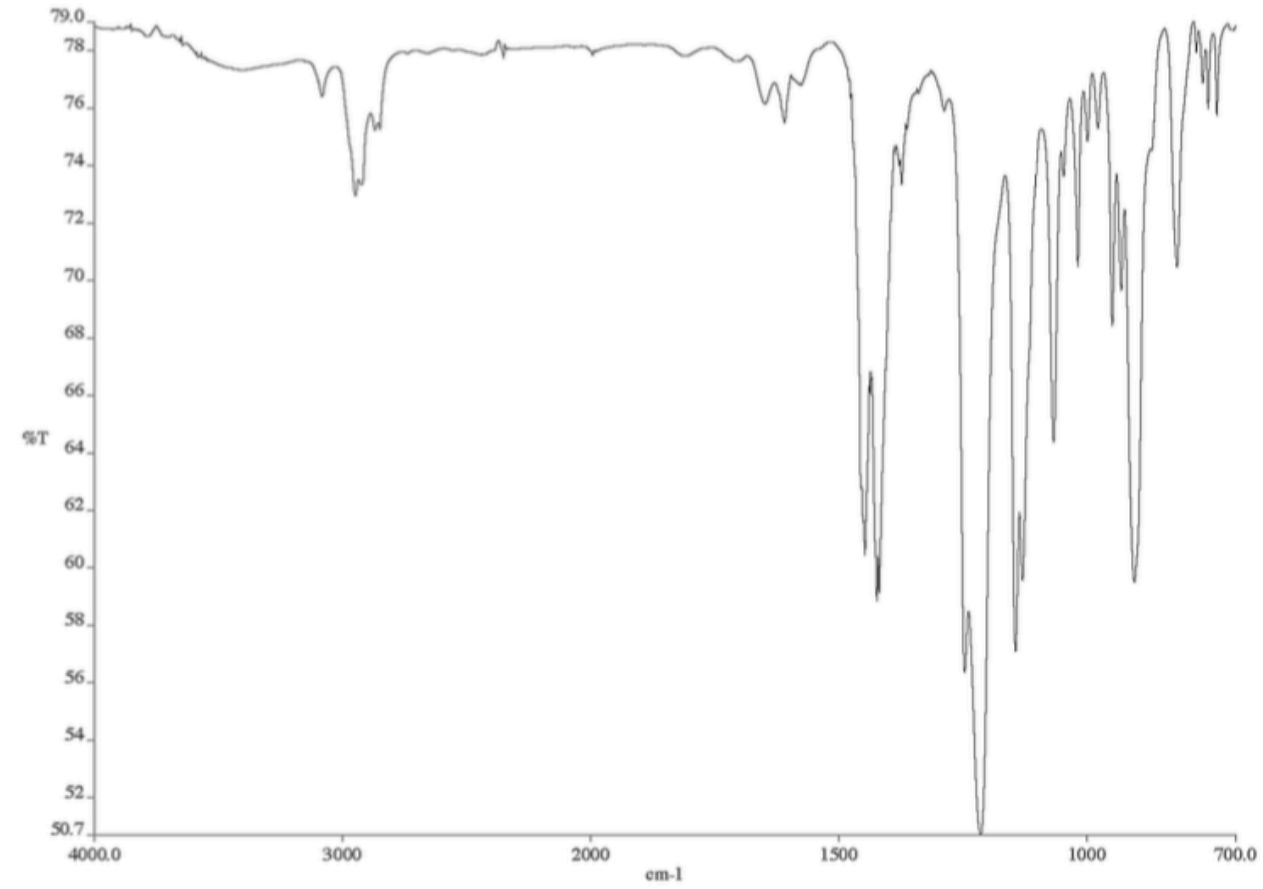

Infrared spectrum (Thin Film, $\mathrm{NaCl}$ ) of compound 27.

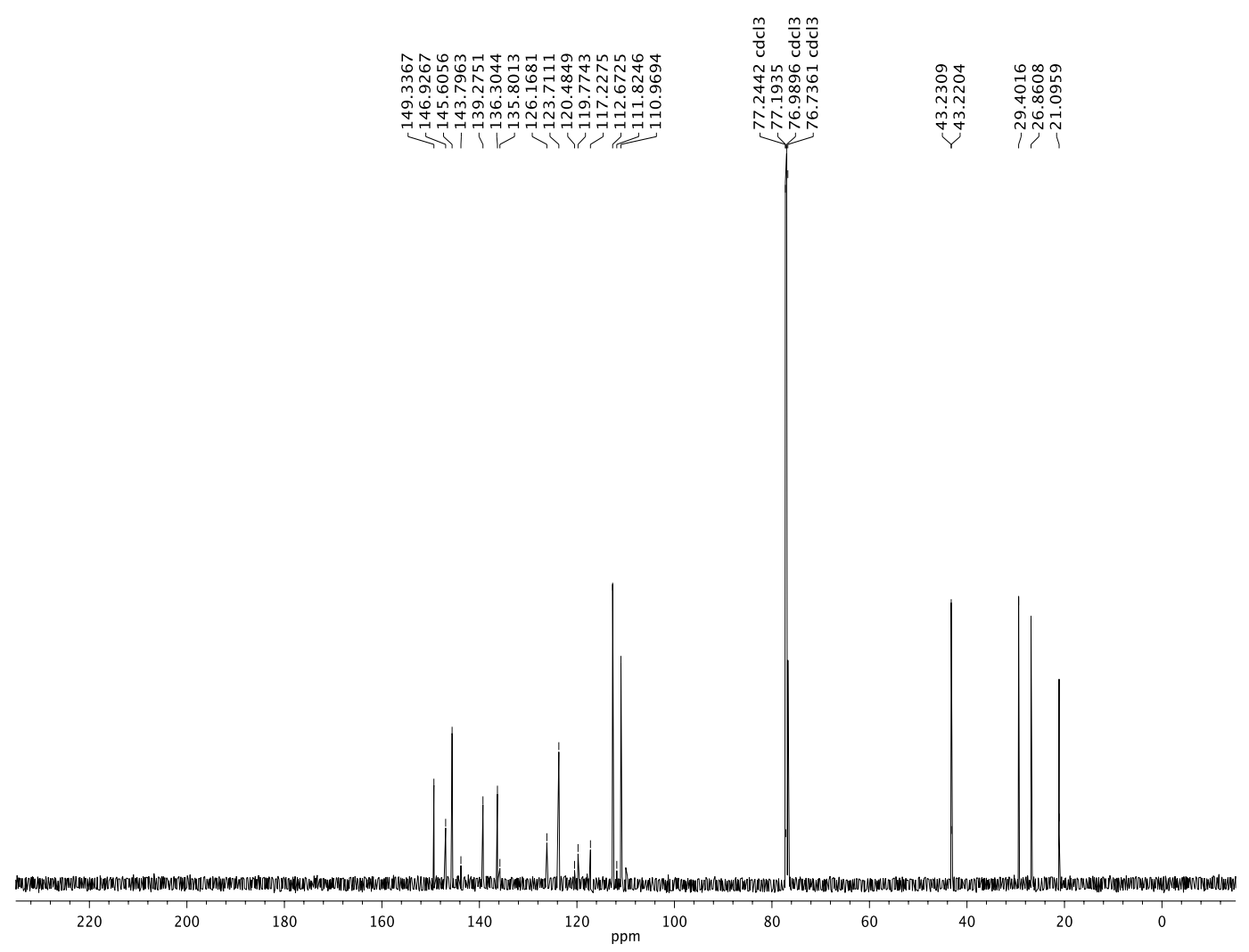

${ }^{13} \mathrm{C}$ NMR (126 MHz, $\mathrm{CDCl}_{3}$ ) of compound 27. 


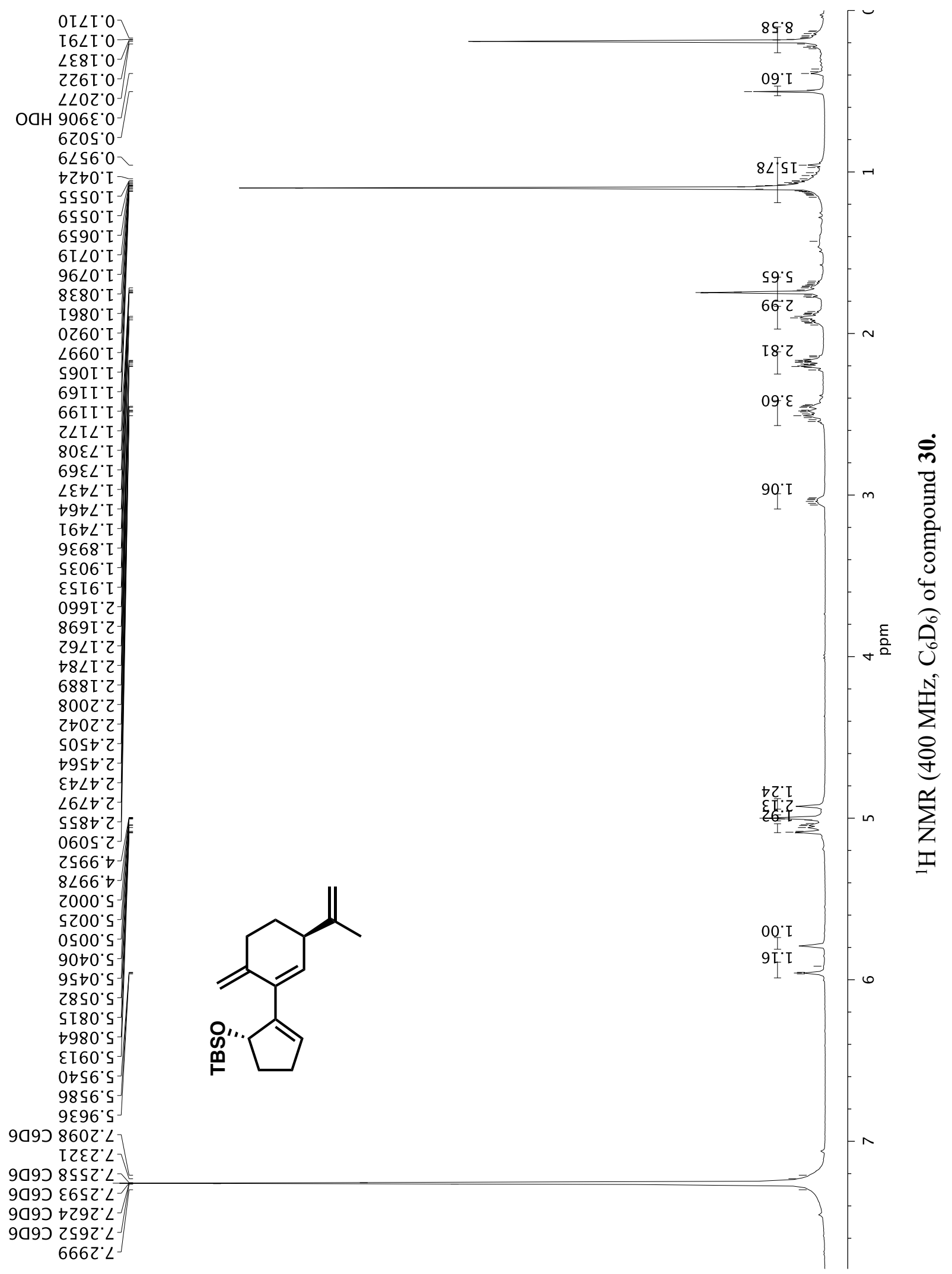



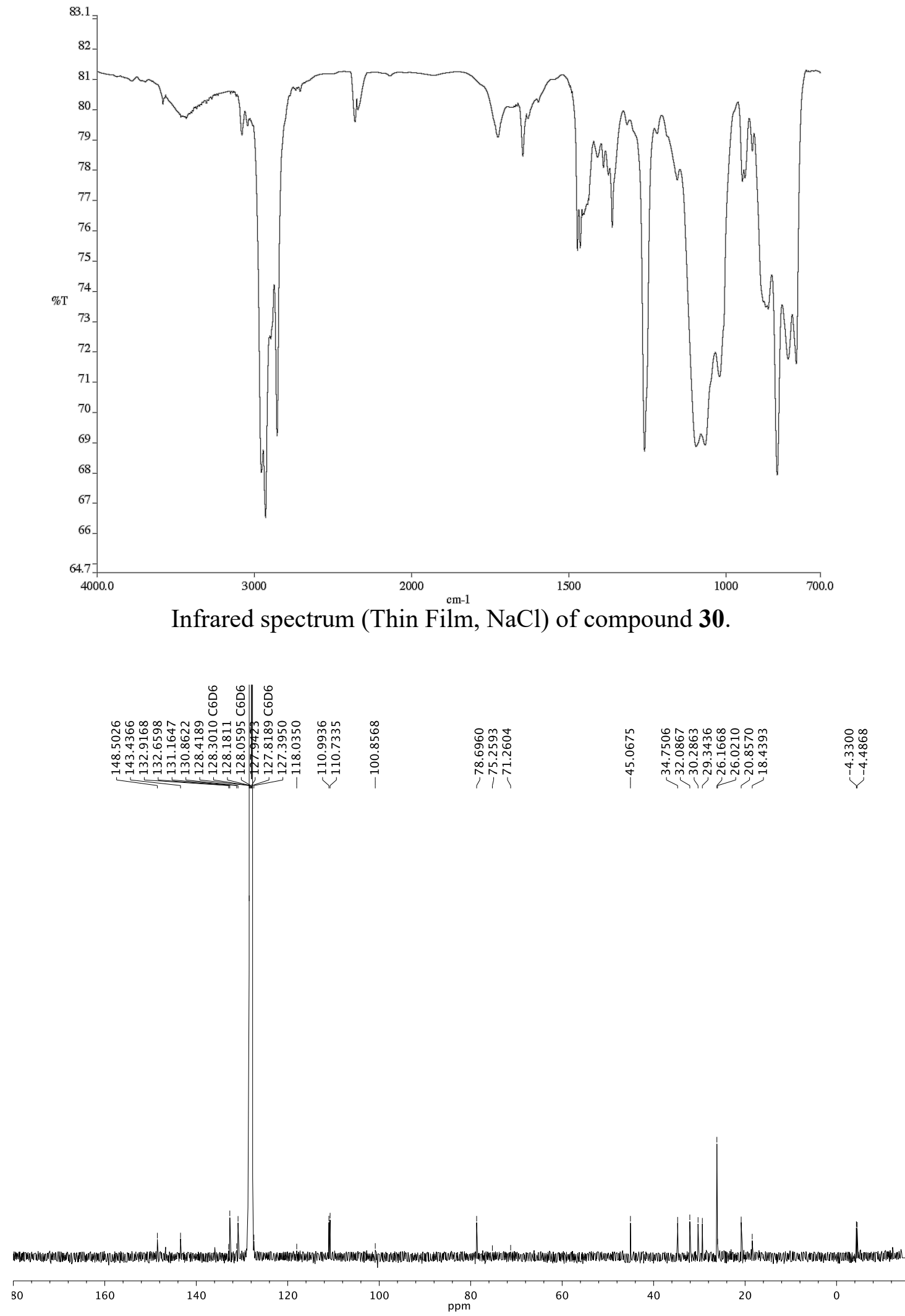

${ }^{13} \mathrm{C}$ NMR (101 MHz, $\left.\mathrm{C}_{6} \mathrm{D}_{6}\right)$ of compound 30. 

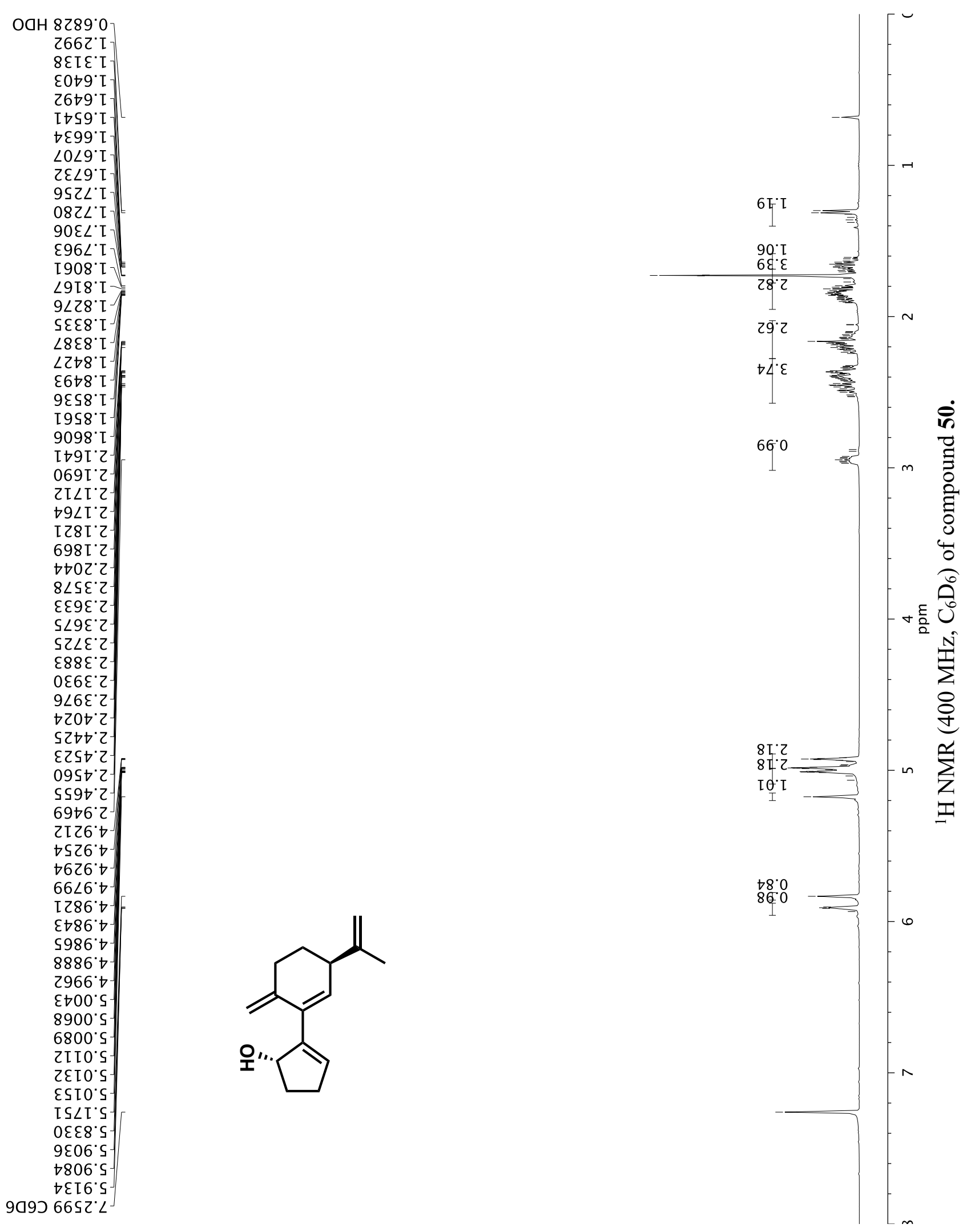

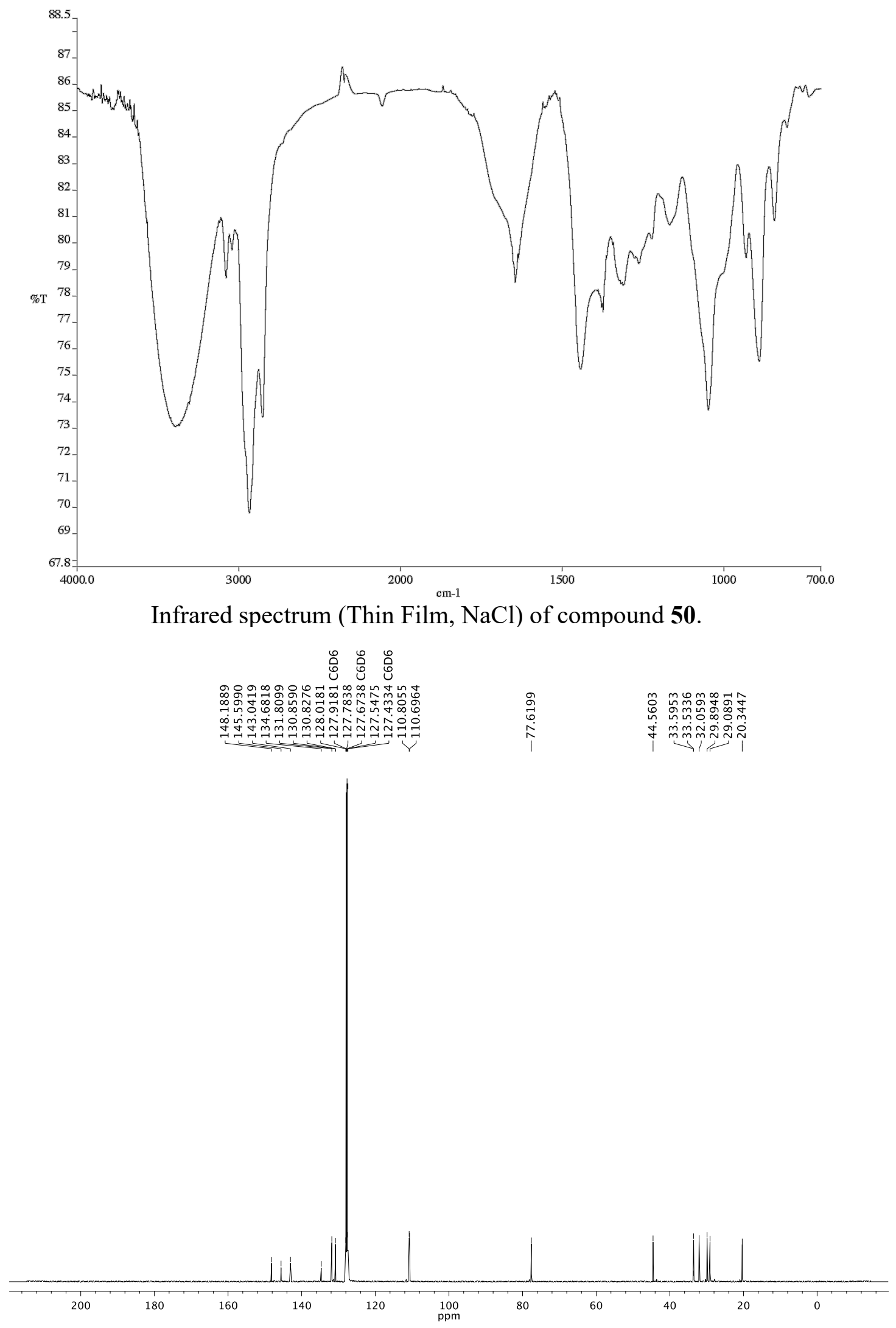

${ }^{13} \mathrm{C}$ NMR (101 MHz, $\mathrm{C}_{6} \mathrm{D}_{6}$ ) of compound $\mathbf{5 0}$. 


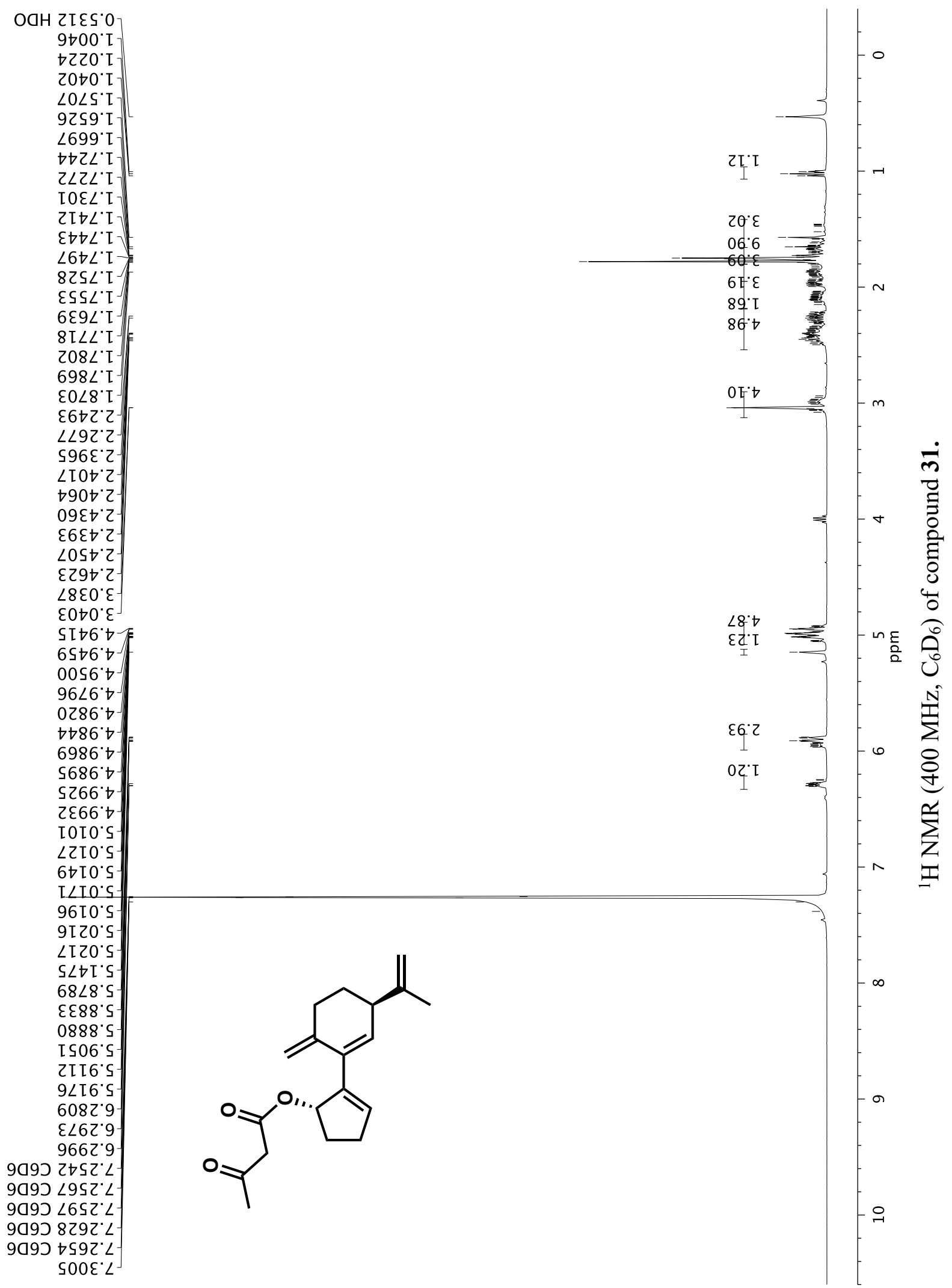




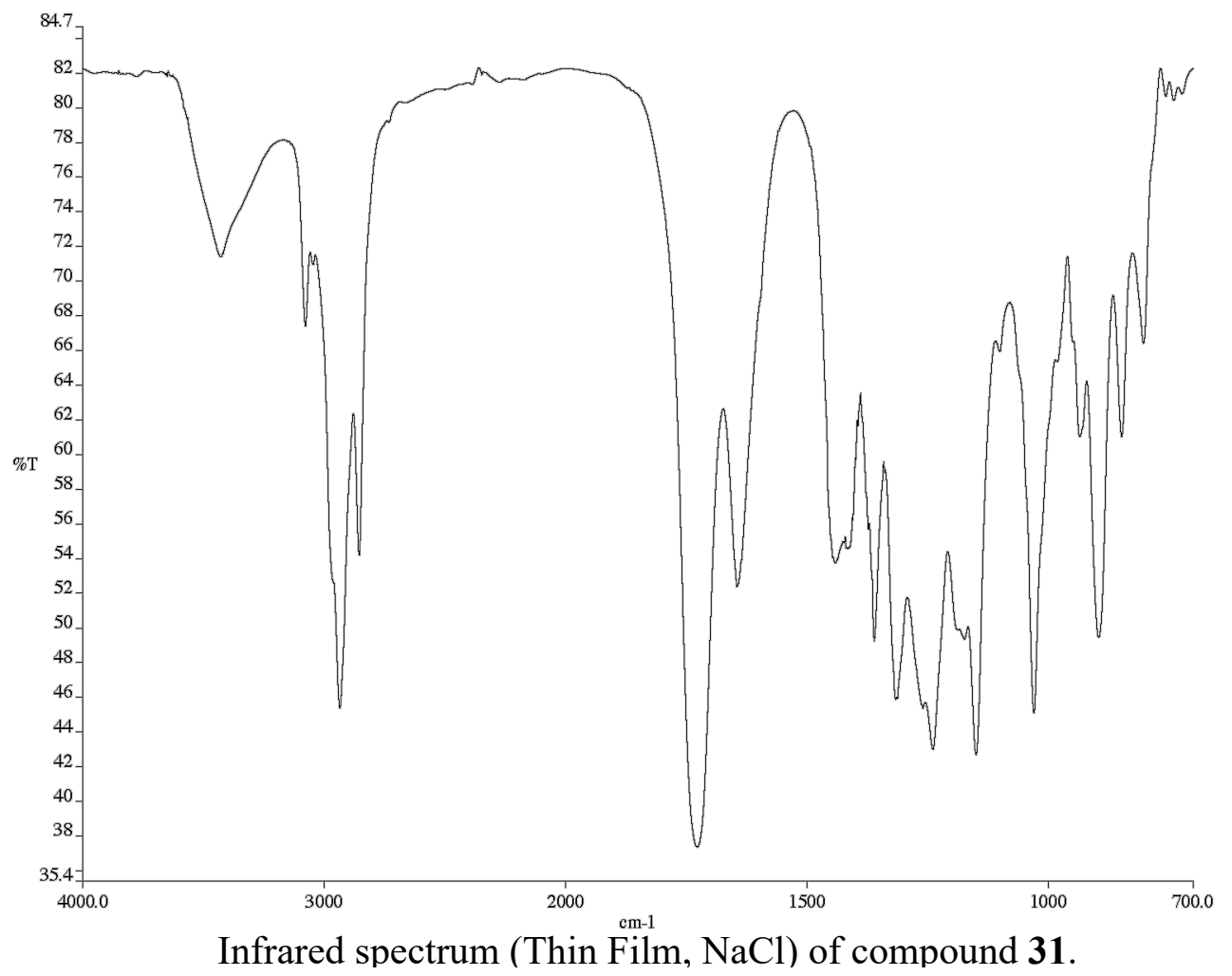

\begin{tabular}{|c|c|c|c|c|c|c|}
\hline 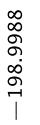 & 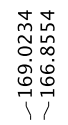 & 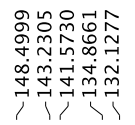 & $\begin{array}{l}\underset{n}{n} \\
\stackrel{-}{-1} \\
- \\
-1 \\
-1\end{array}$ & $\begin{array}{c}n \\
\stackrel{0}{0} \\
\stackrel{1}{i} \\
\infty \\
1\end{array}$ & 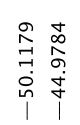 & 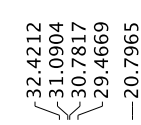 \\
\hline
\end{tabular}

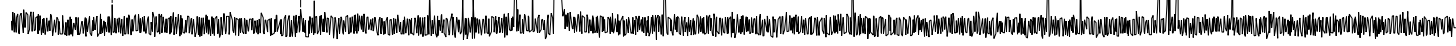

${ }^{13} \mathrm{C}$ NMR (101 MHz, $\mathrm{C}_{6} \mathrm{D}_{6}$ ) of compound 31. 


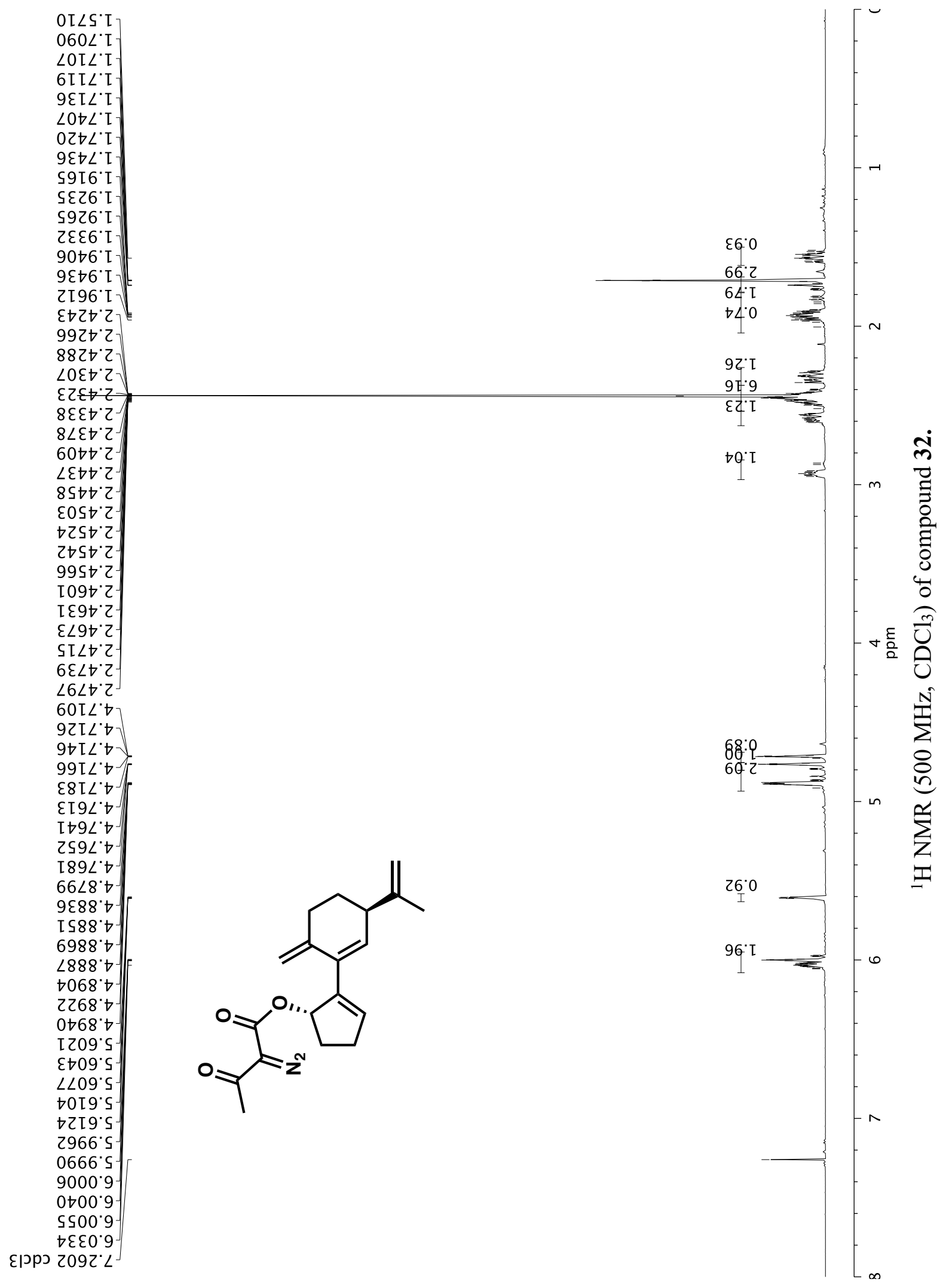



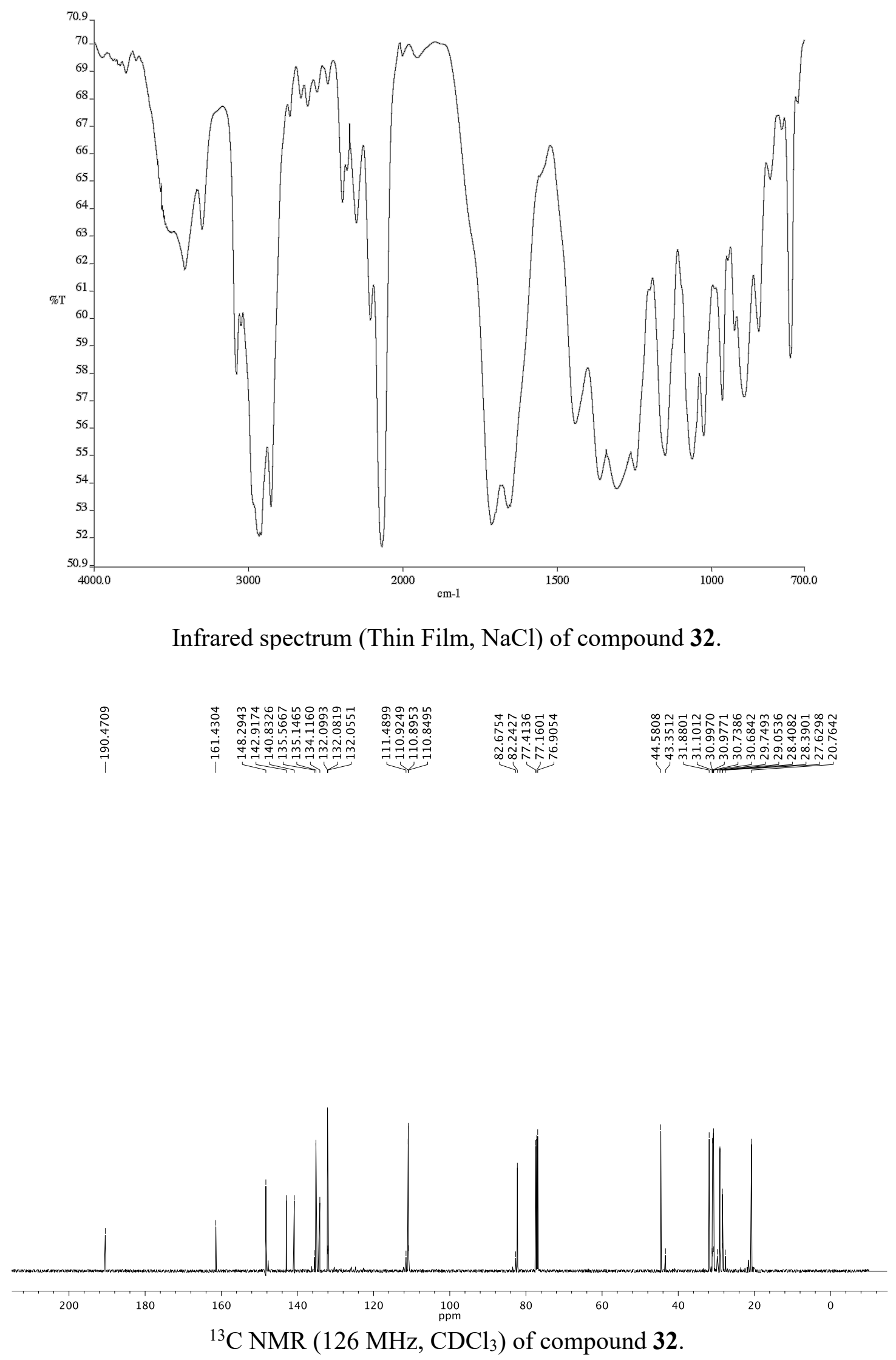


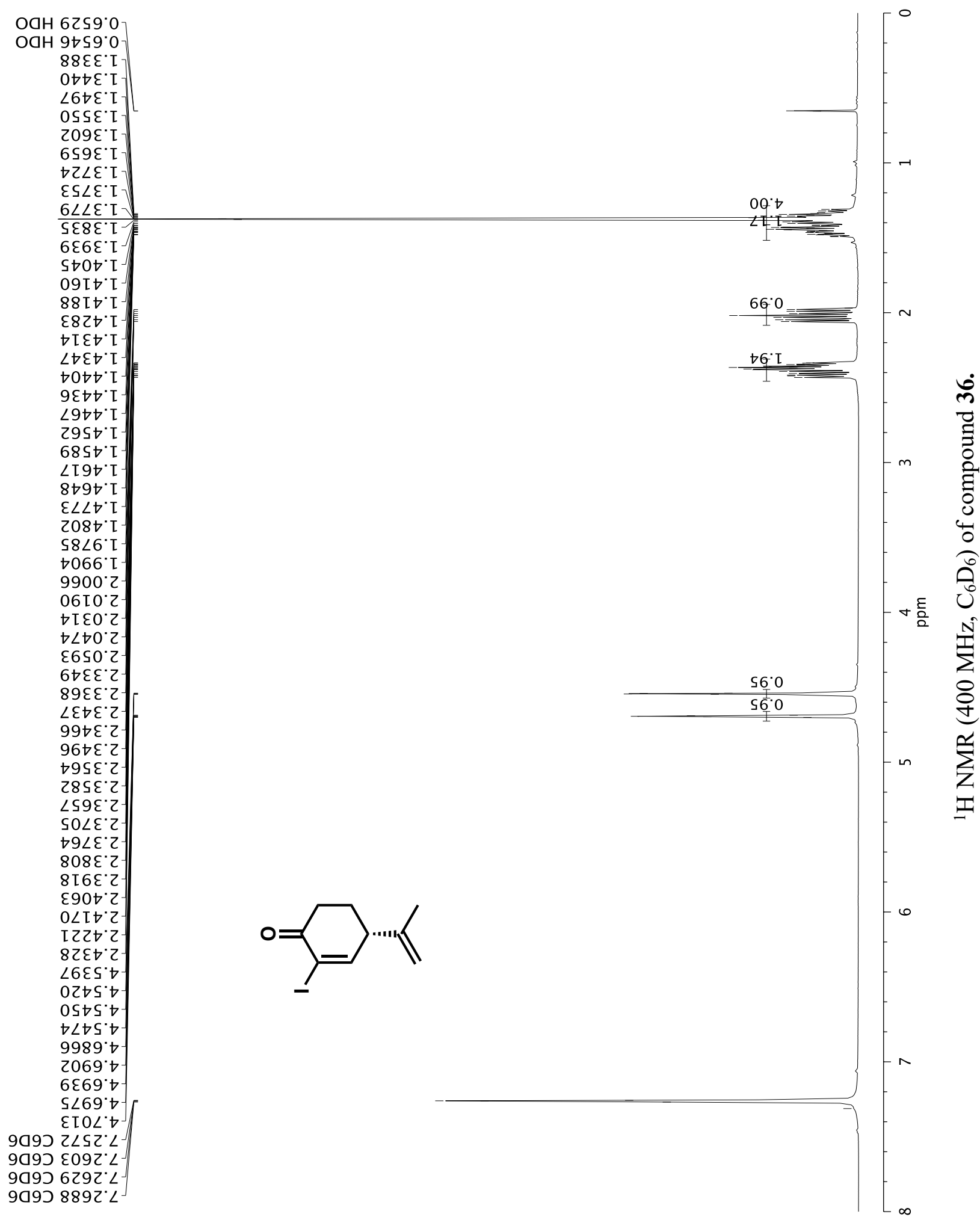



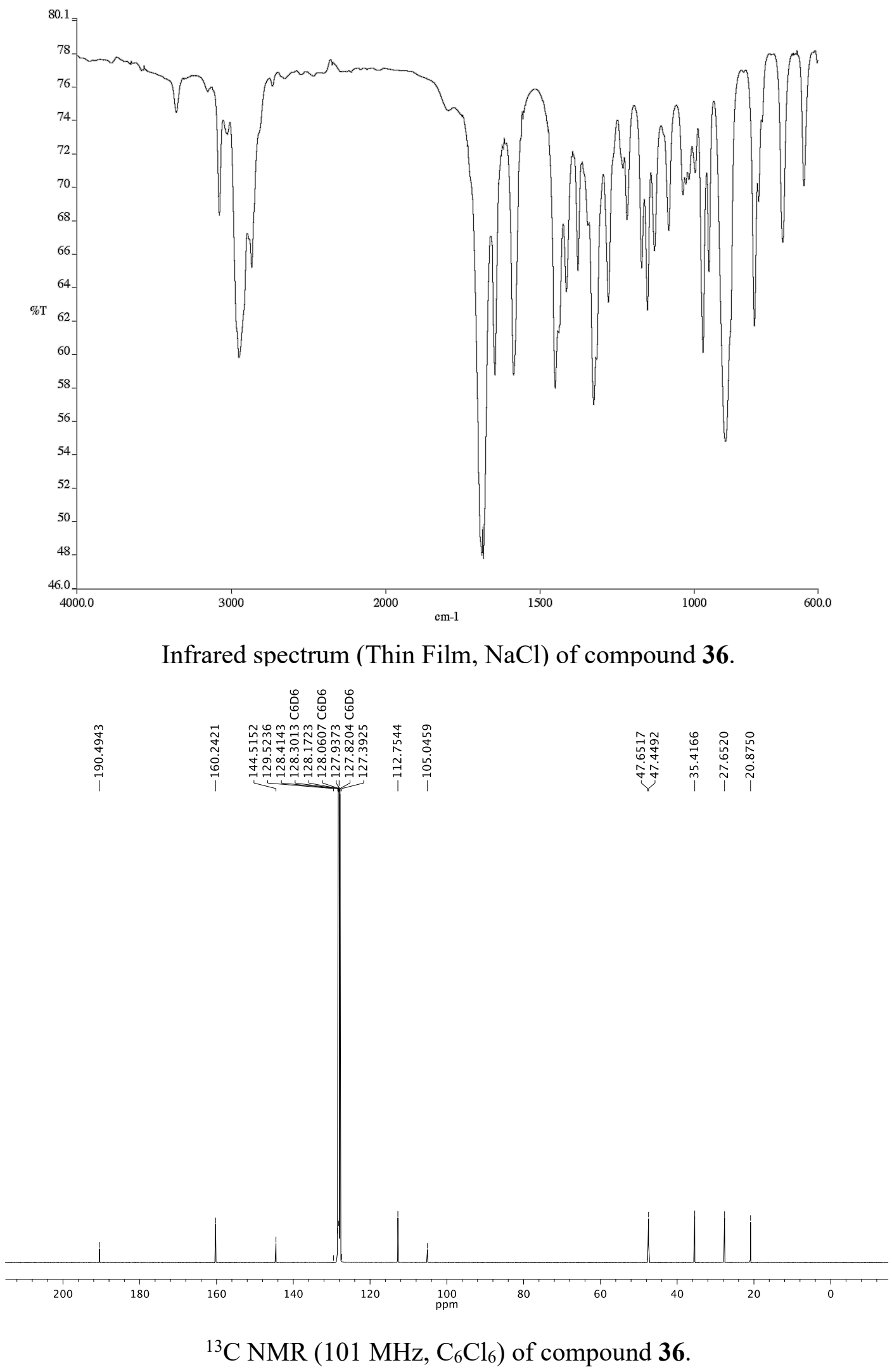


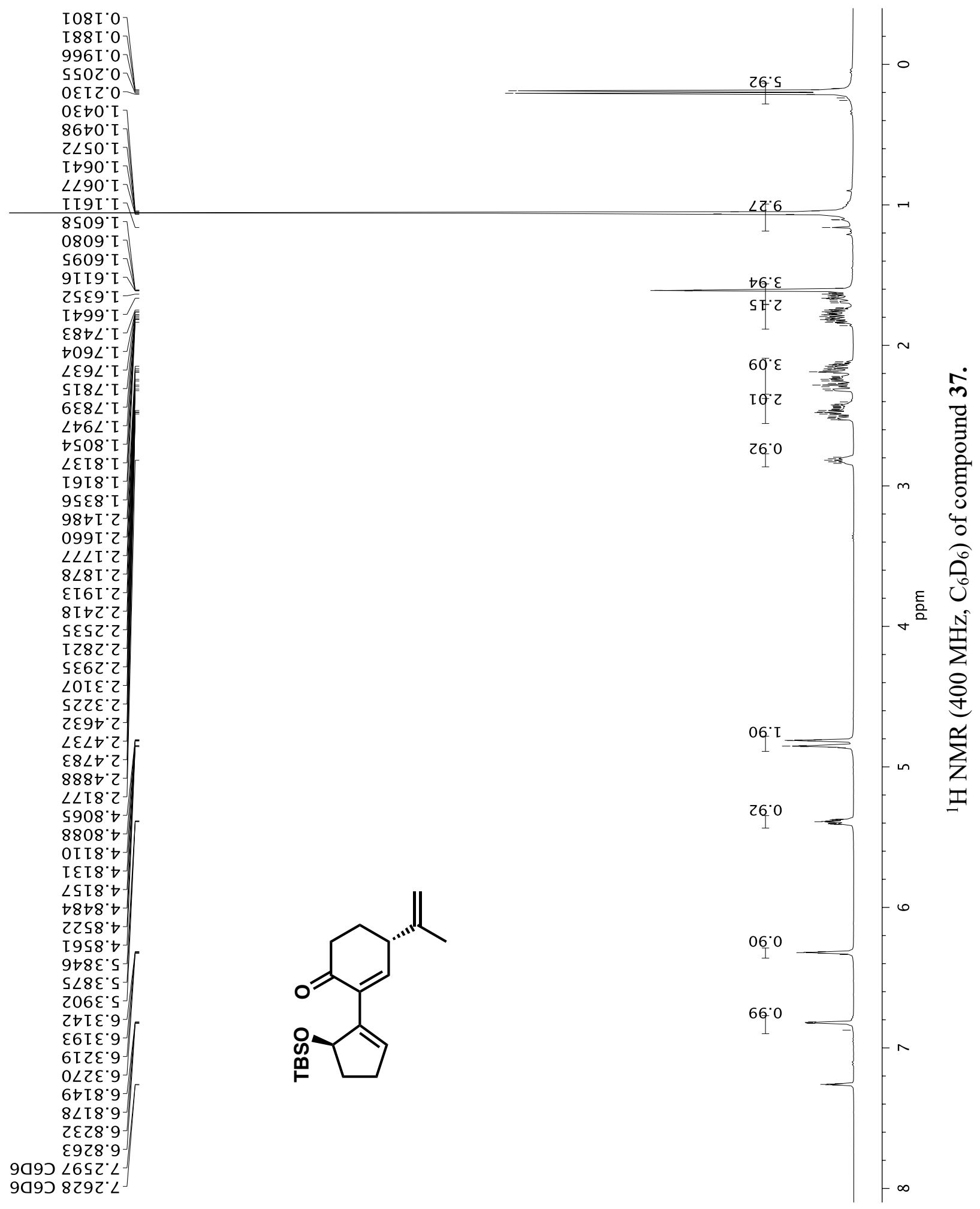



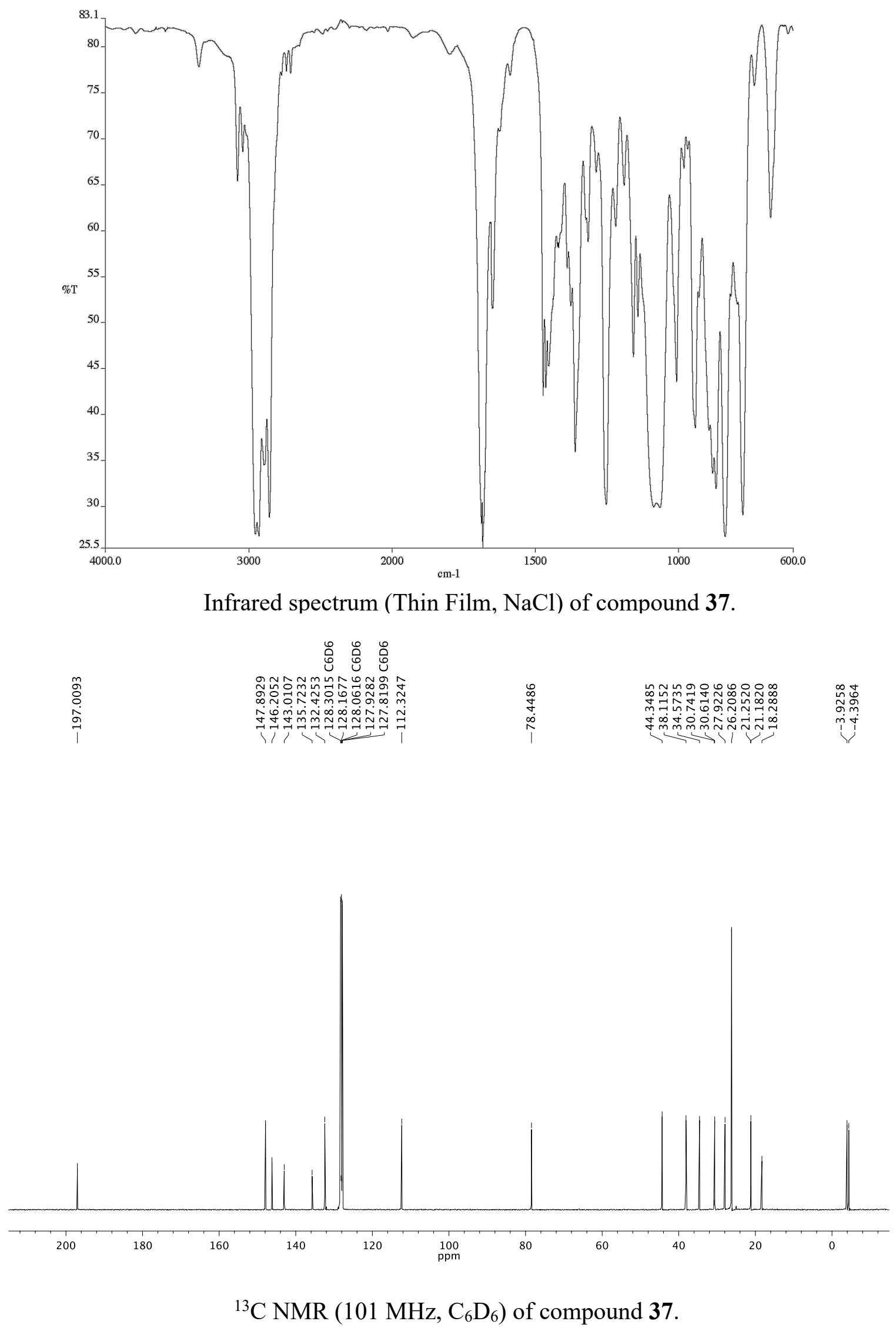


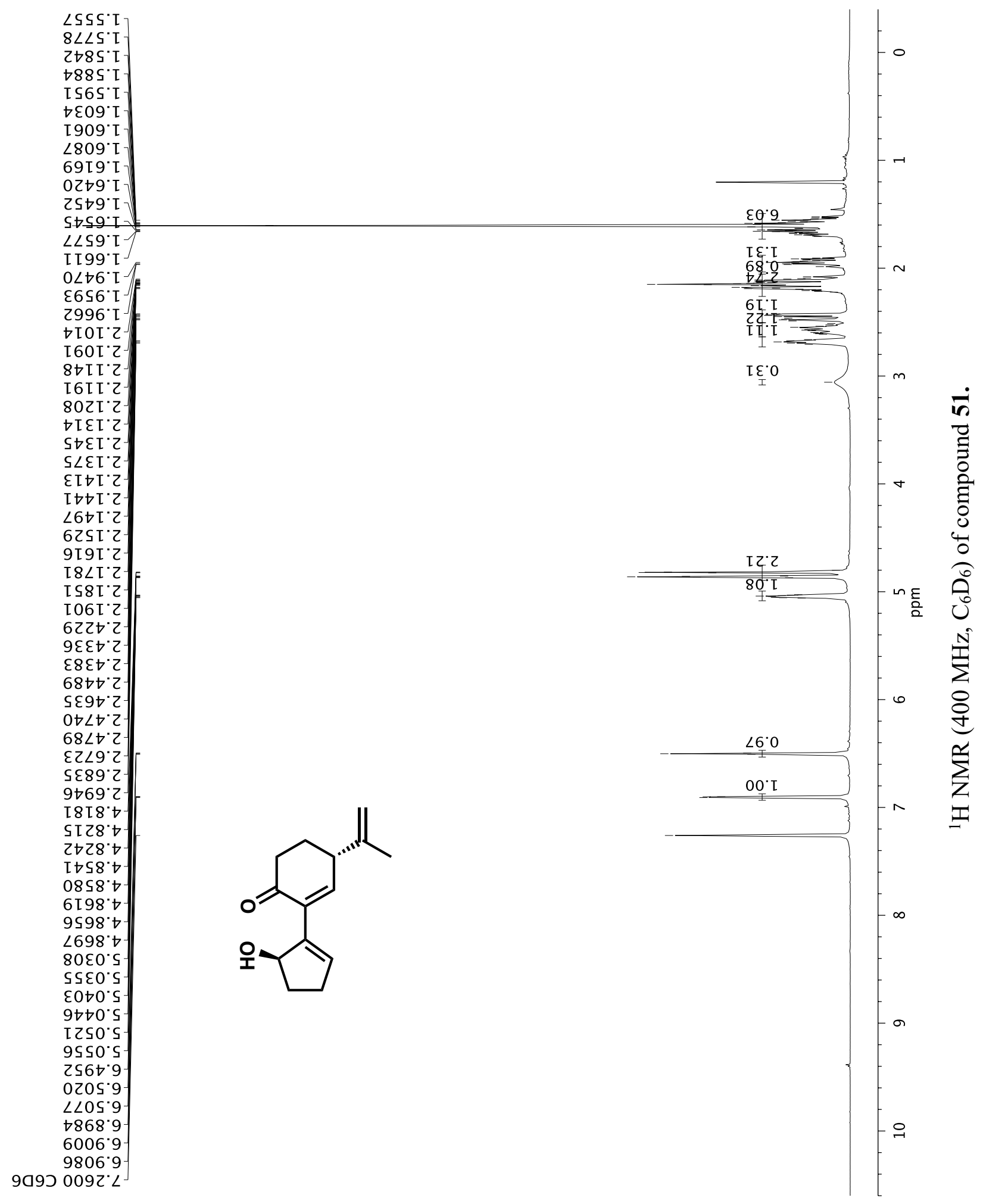




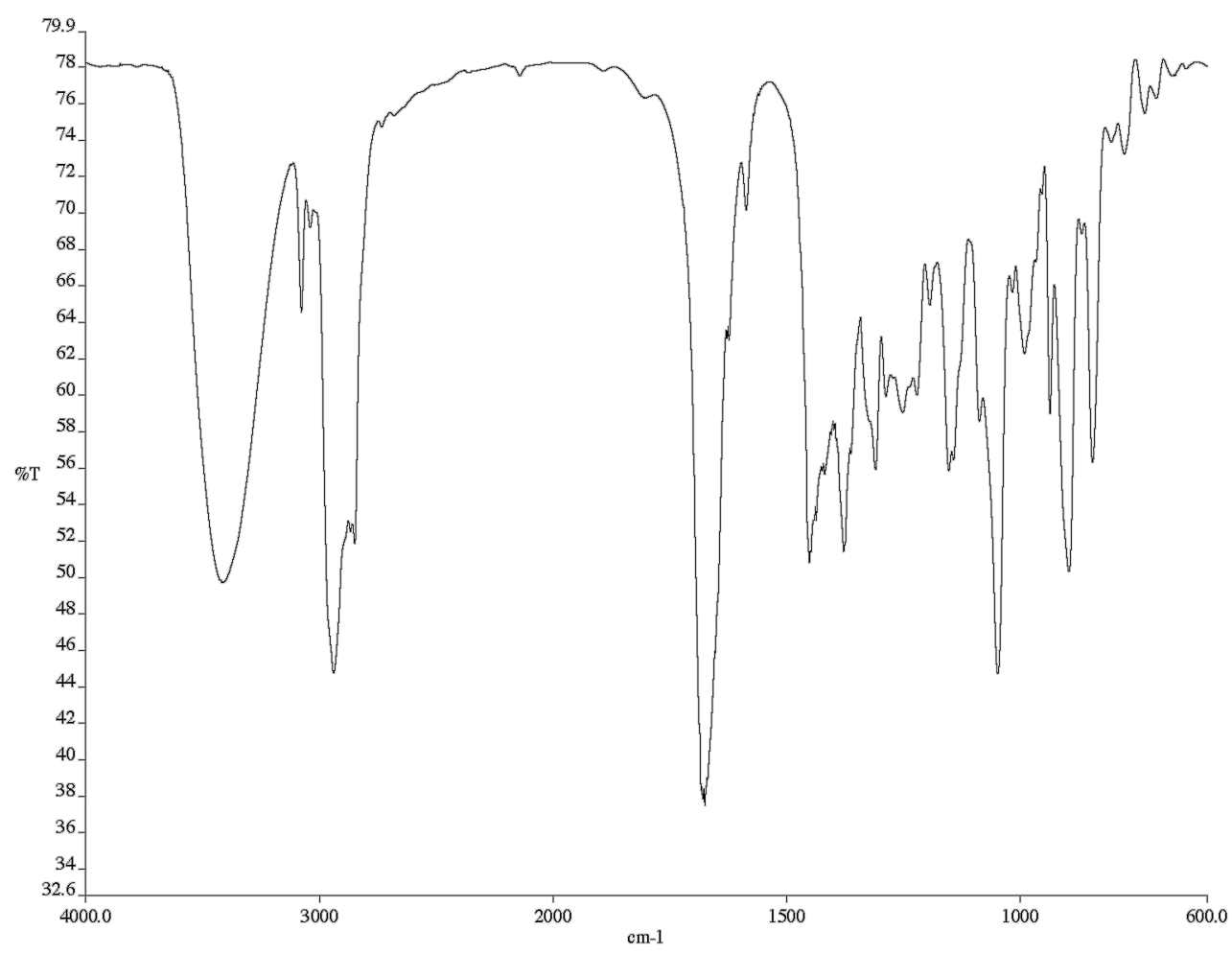

Infrared spectrum (Thin Film, $\mathrm{NaCl}$ ) of compound $\mathbf{5 1}$.

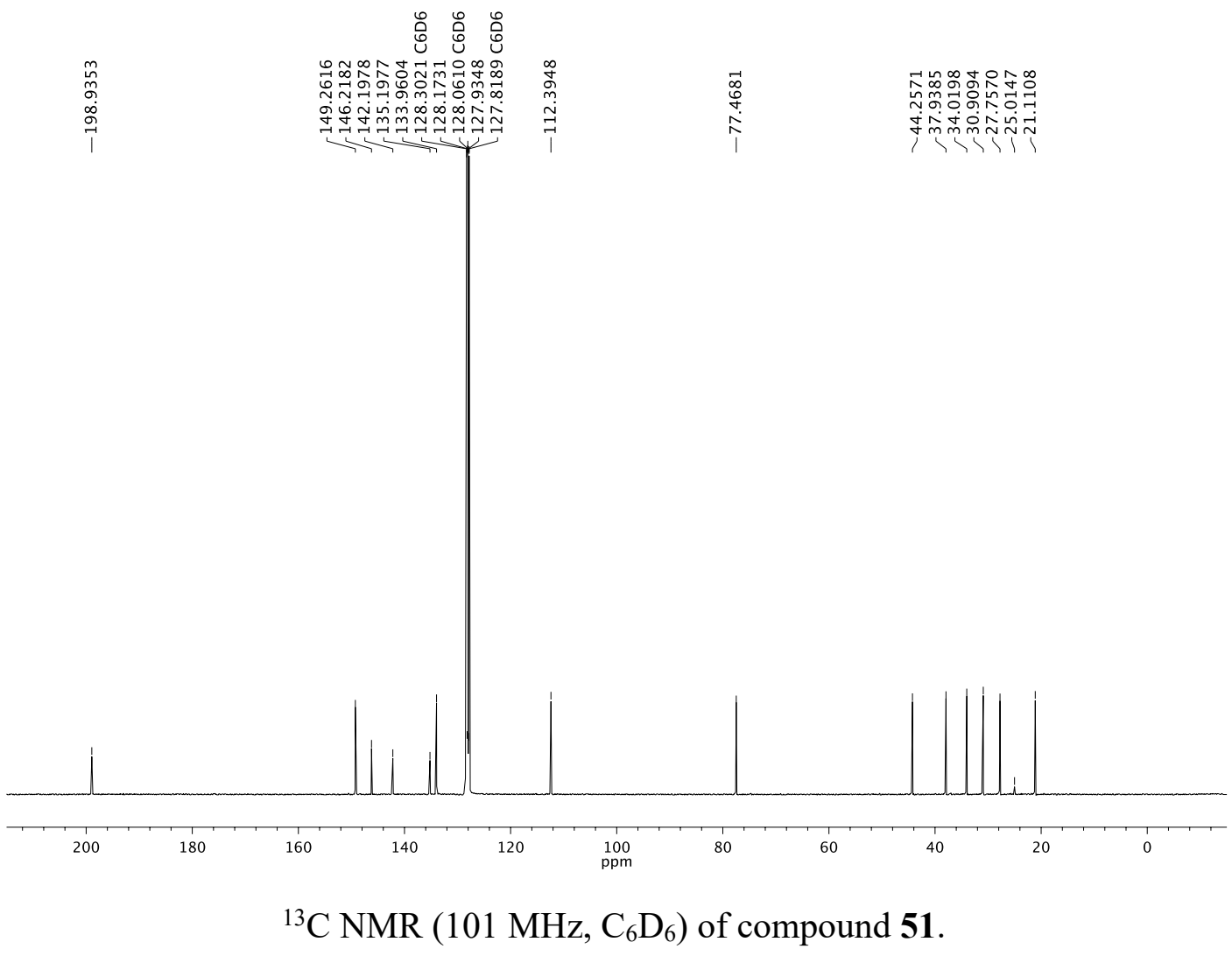




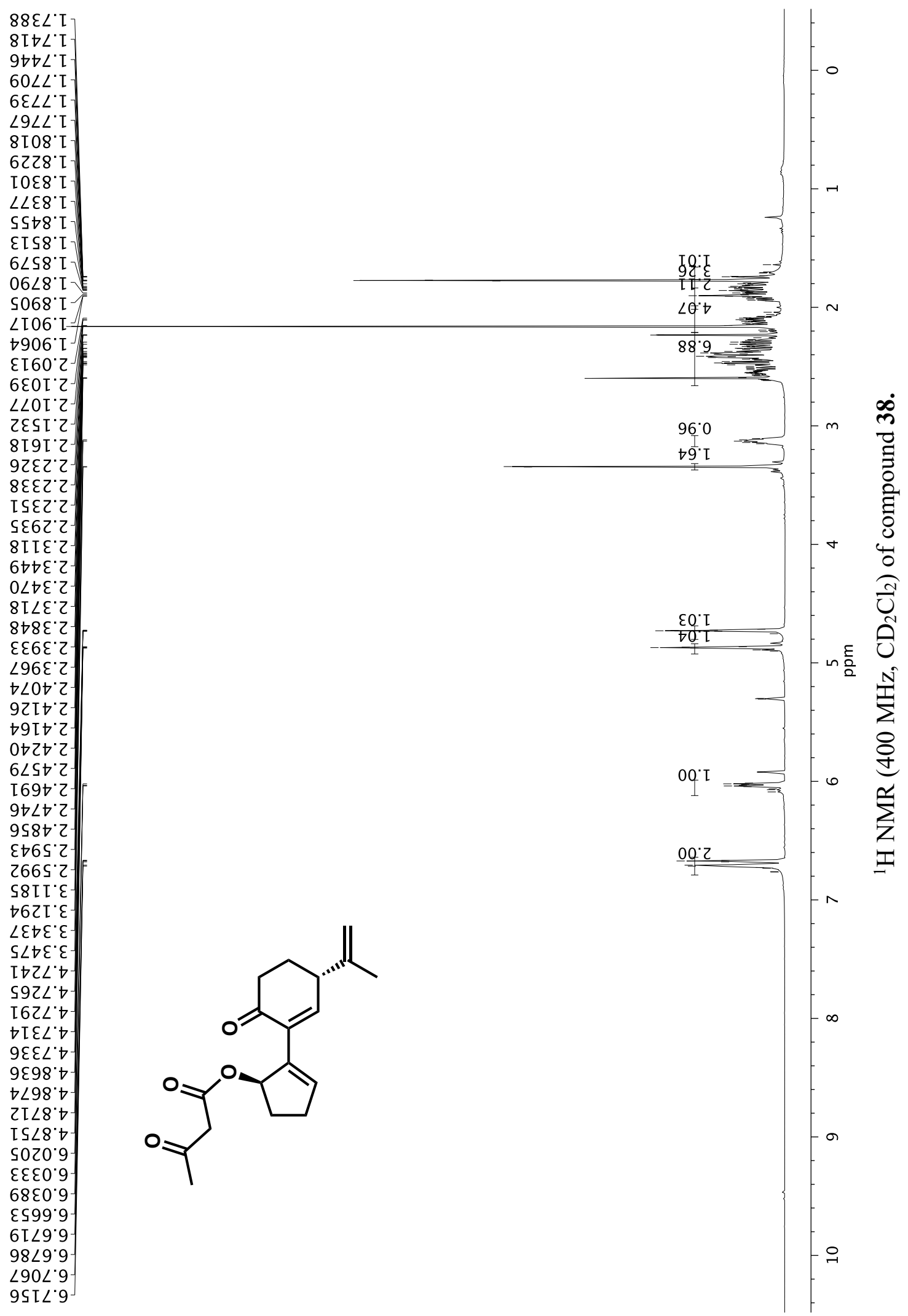



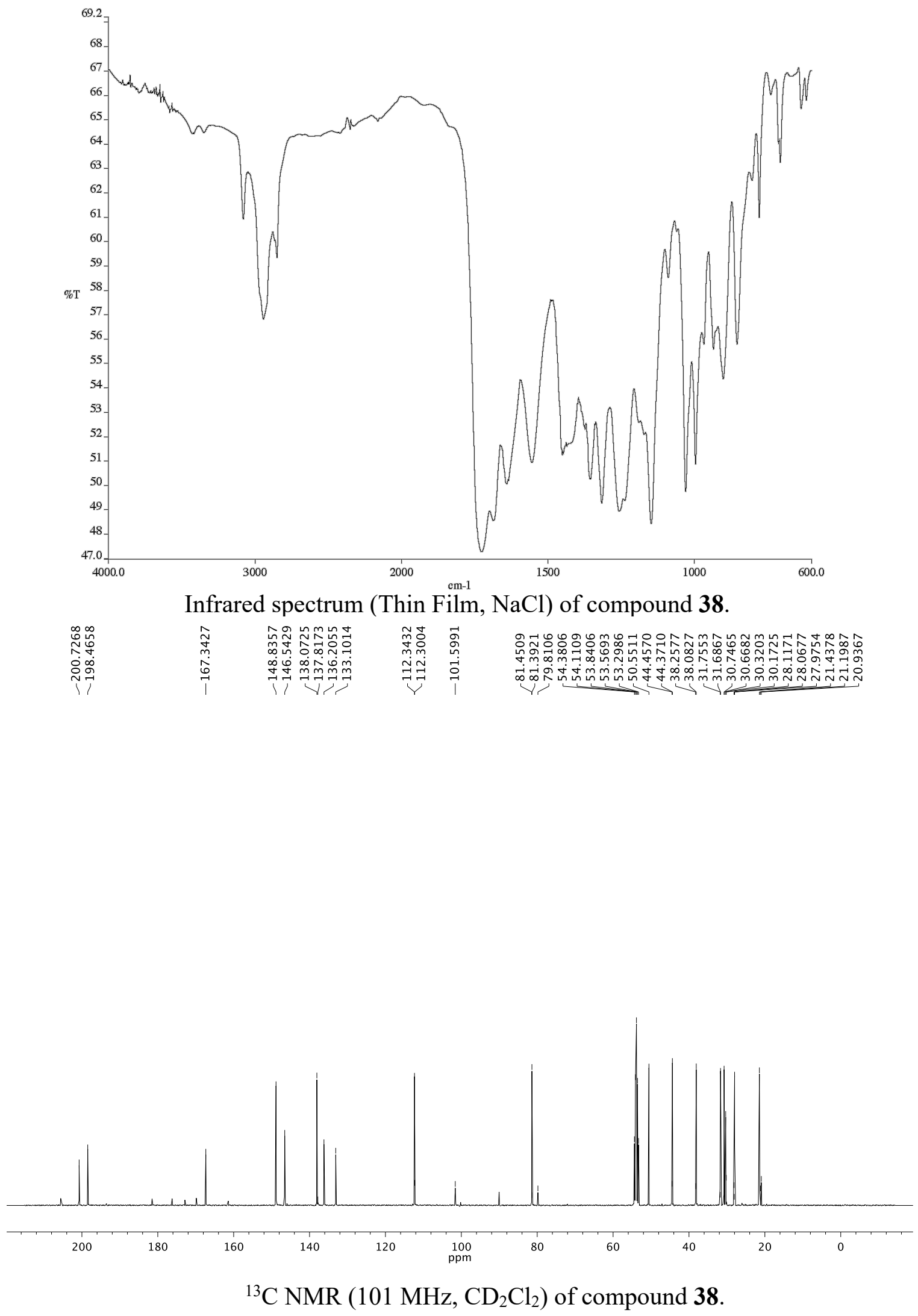


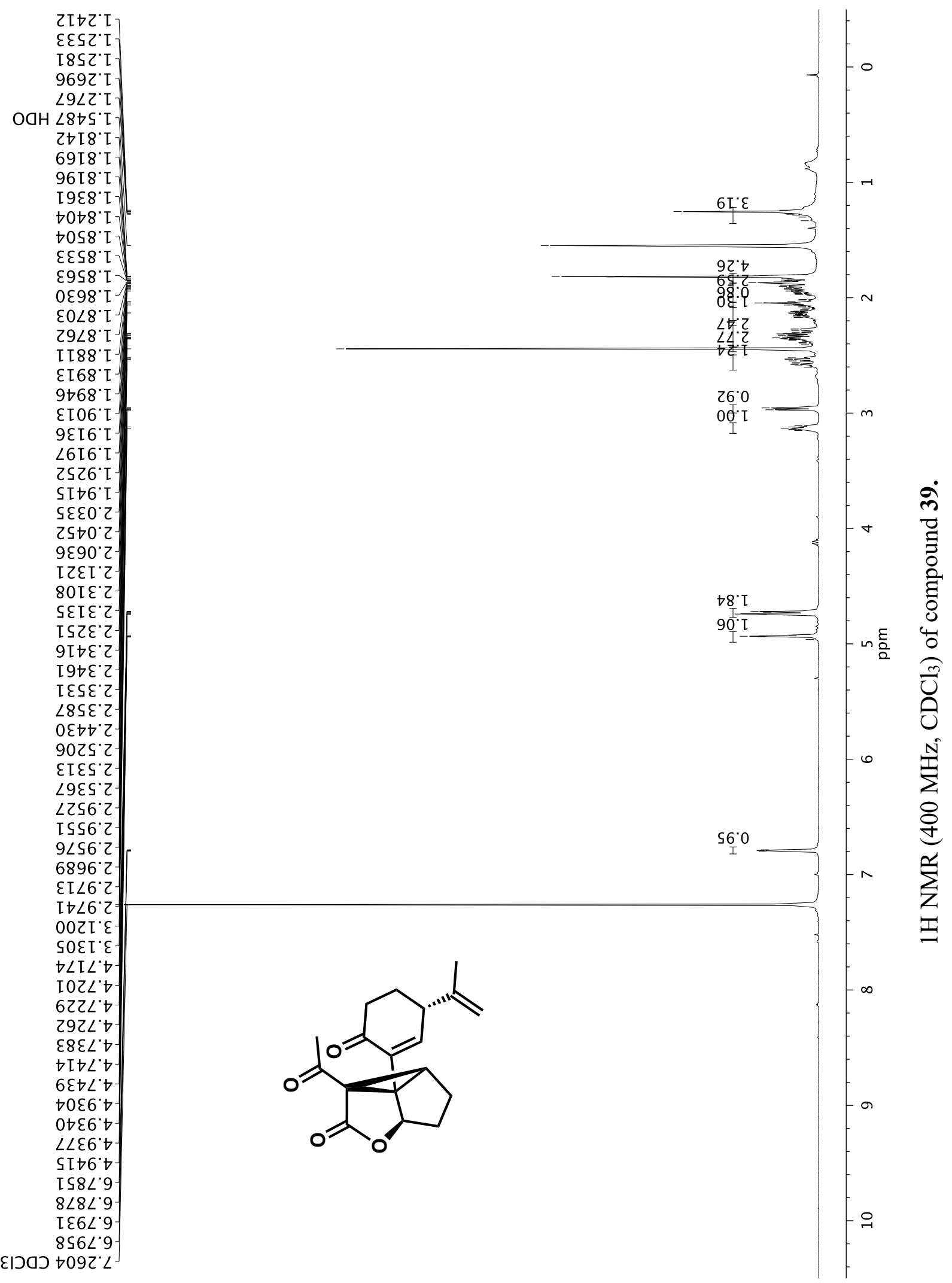




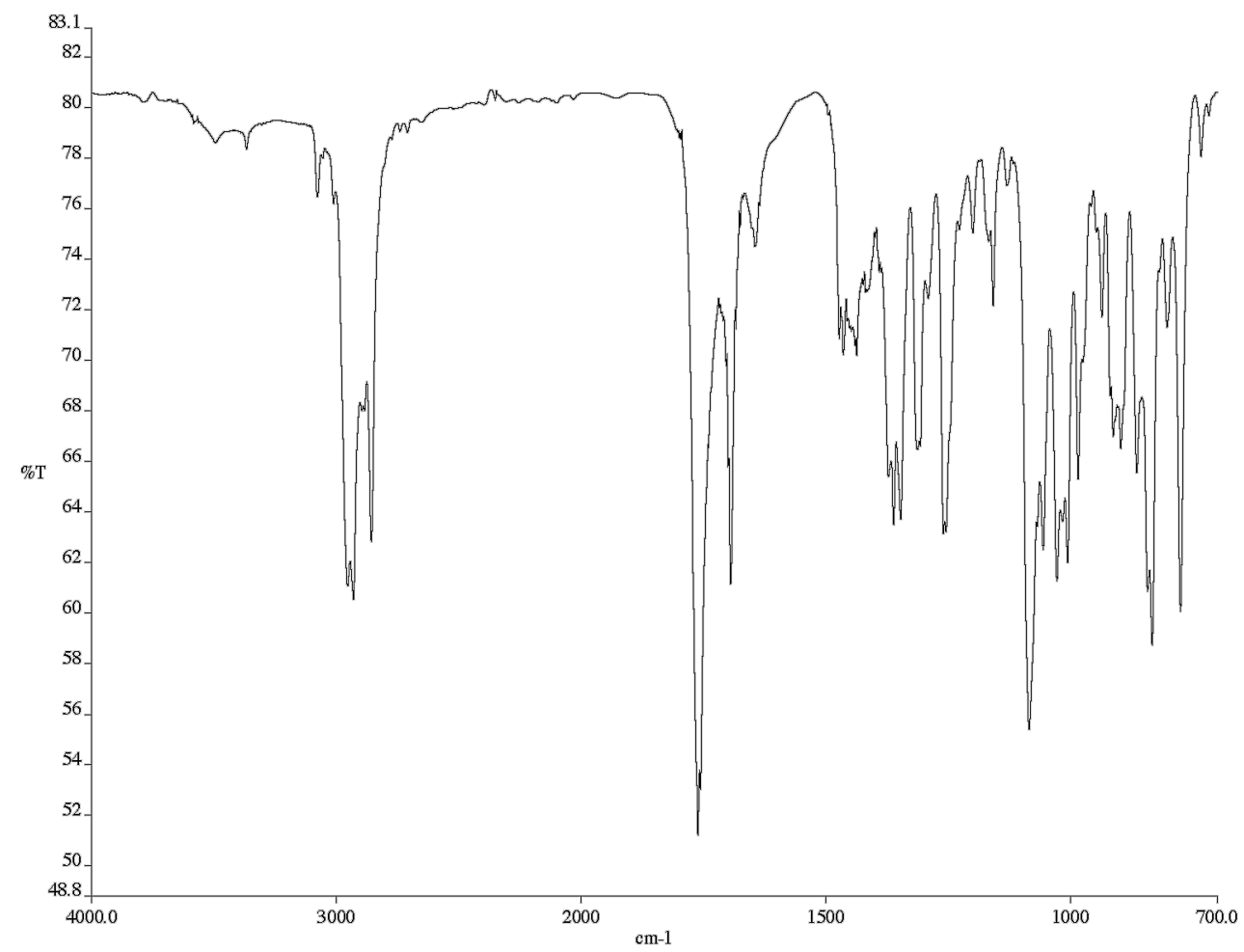

Infrared spectrum (Thin Film, $\mathrm{NaCl}$ ) of compound 39.

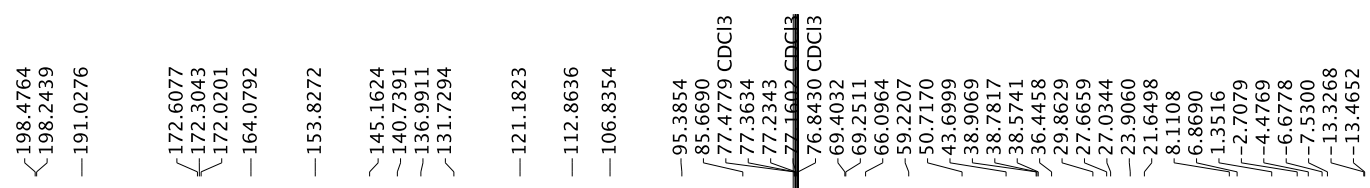

1.5. 160 140 120 100
$\mathrm{ppm}$ 60

${ }^{13} \mathrm{C}$ NMR (101 MHz, $\left.\mathrm{CDCl}_{3}\right)$ of compound 39. 

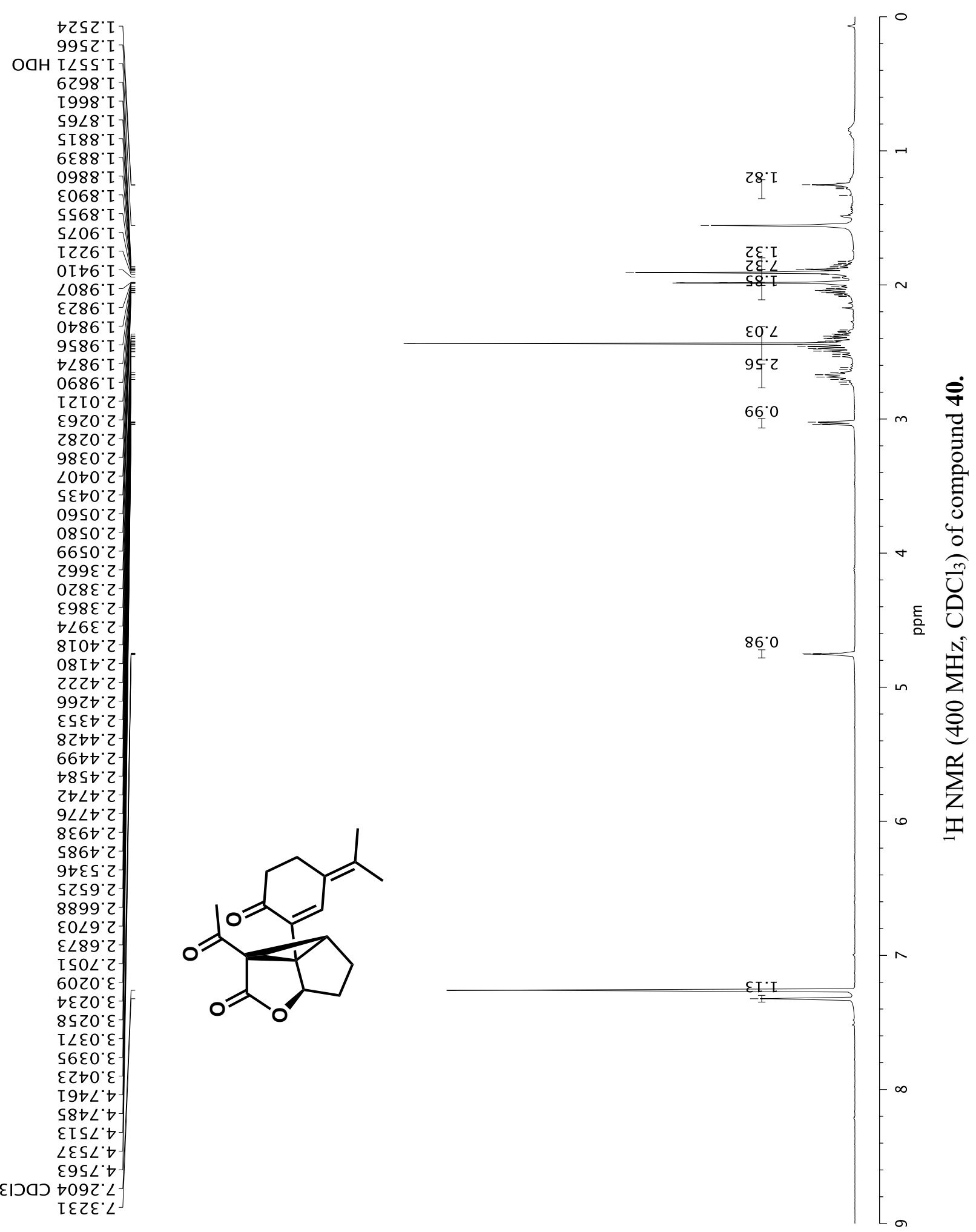


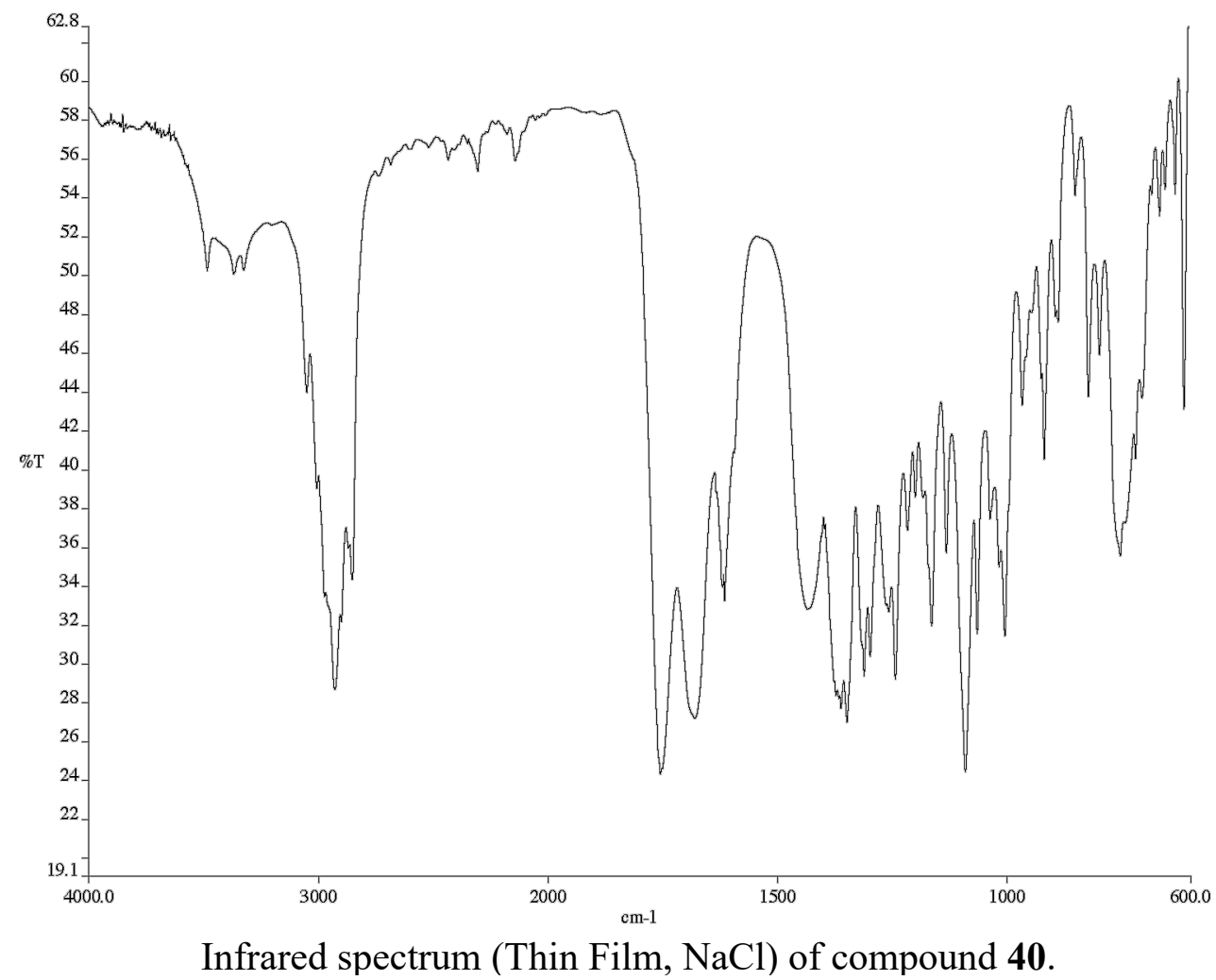

\begin{tabular}{|c|c|c|}
\hline 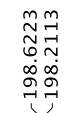 & 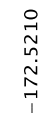 & 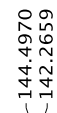 \\
\hline
\end{tabular}

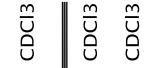

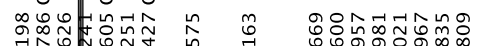

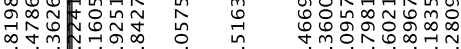

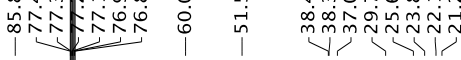

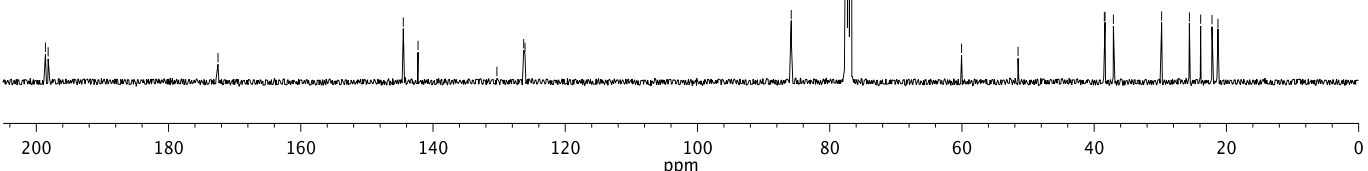

${ }^{13} \mathrm{C}$ NMR (101 MHz, $\left.\mathrm{CDCl}_{3}\right)$ of compound $4 \mathbf{0}$. 

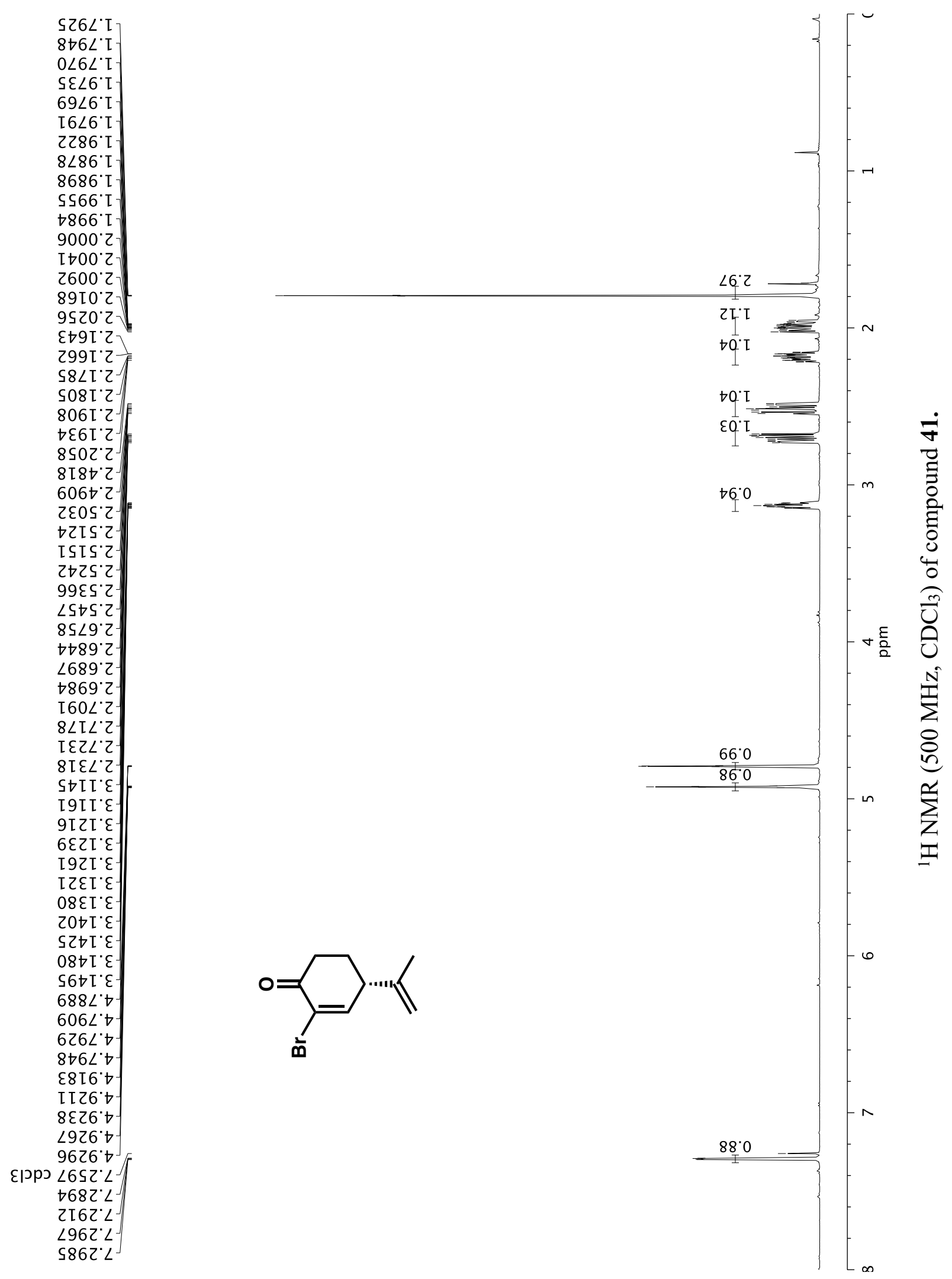

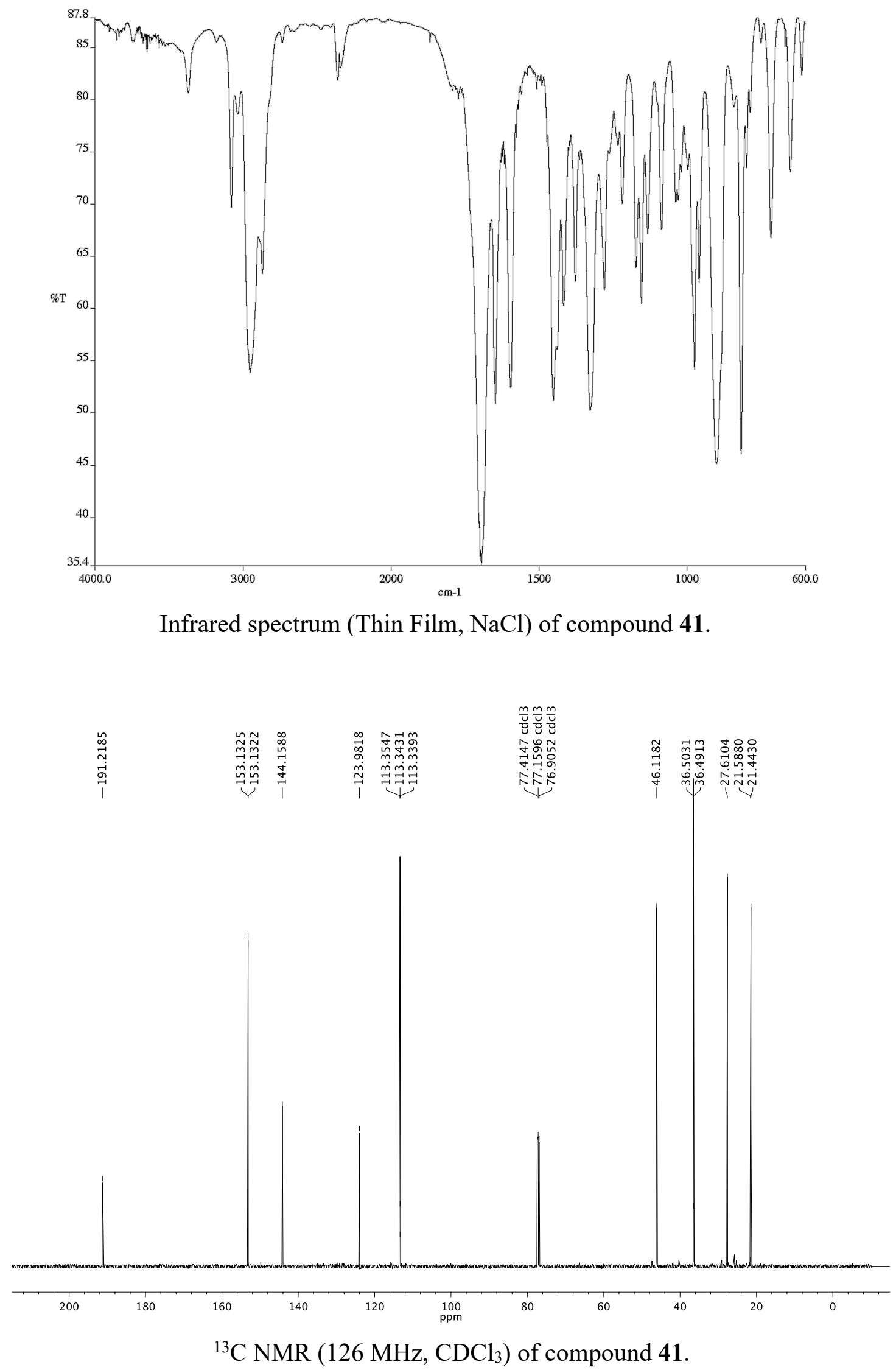


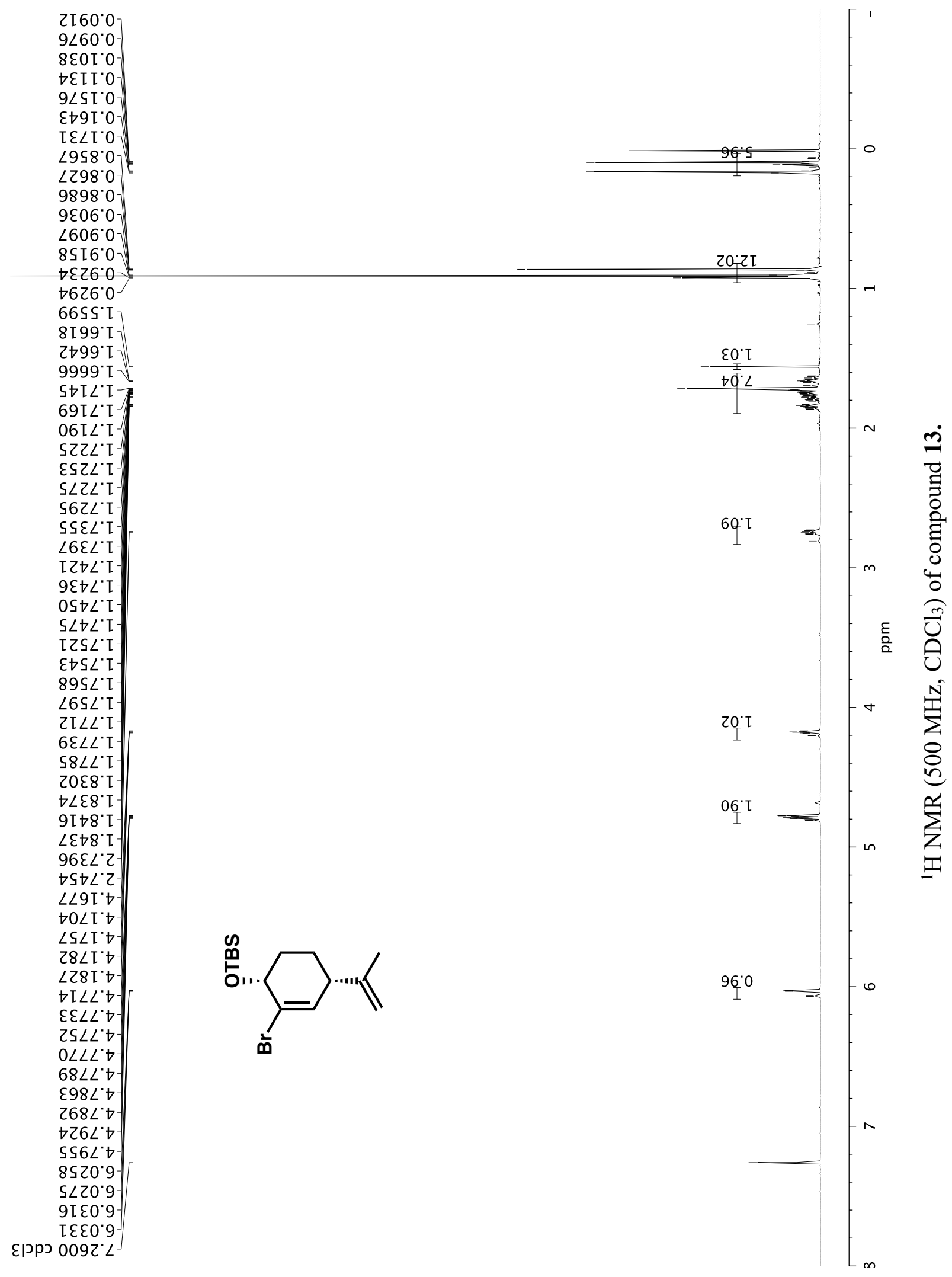




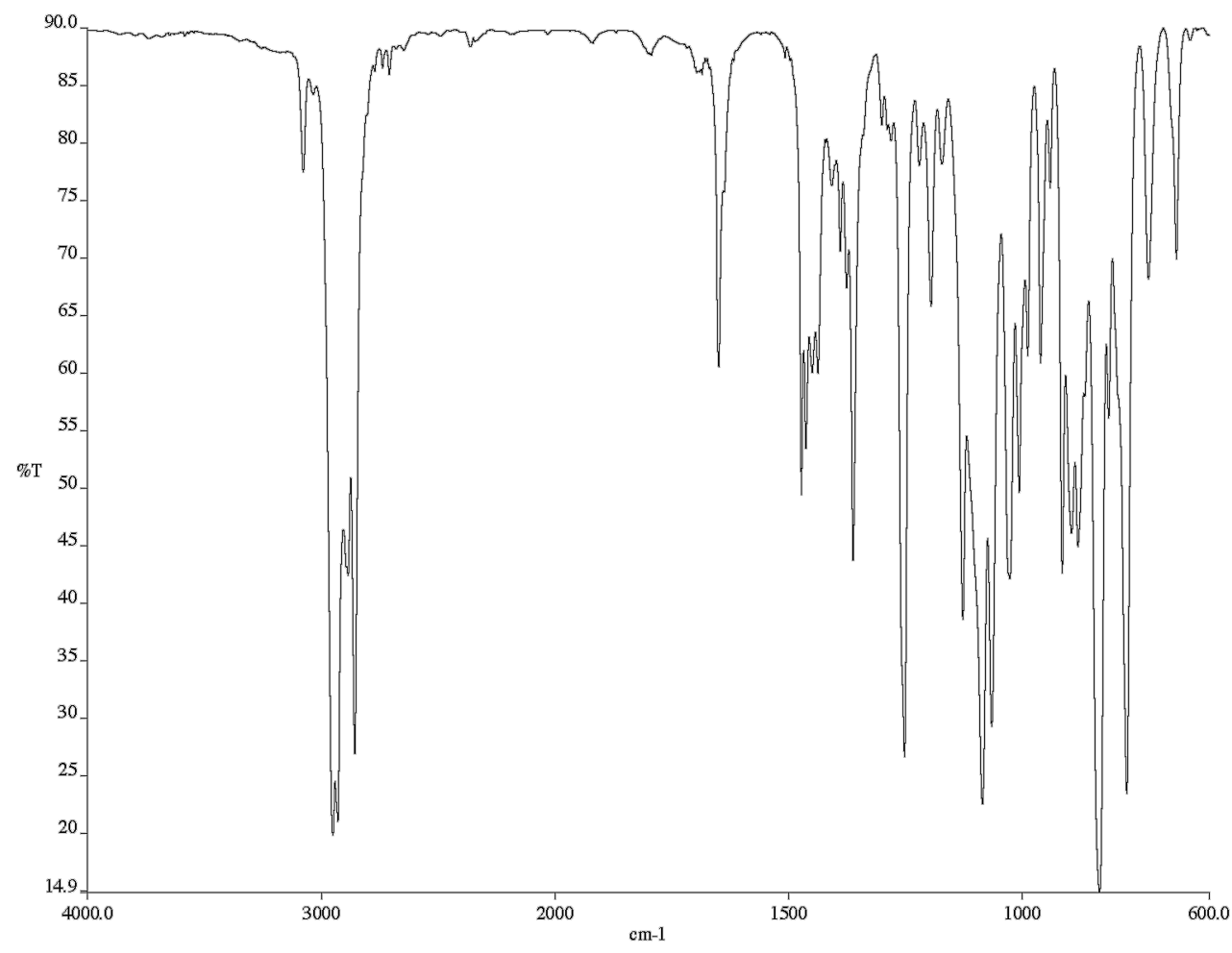

Infrared spectrum (Thin Film, $\mathrm{NaCl}$ ) of compound 13.
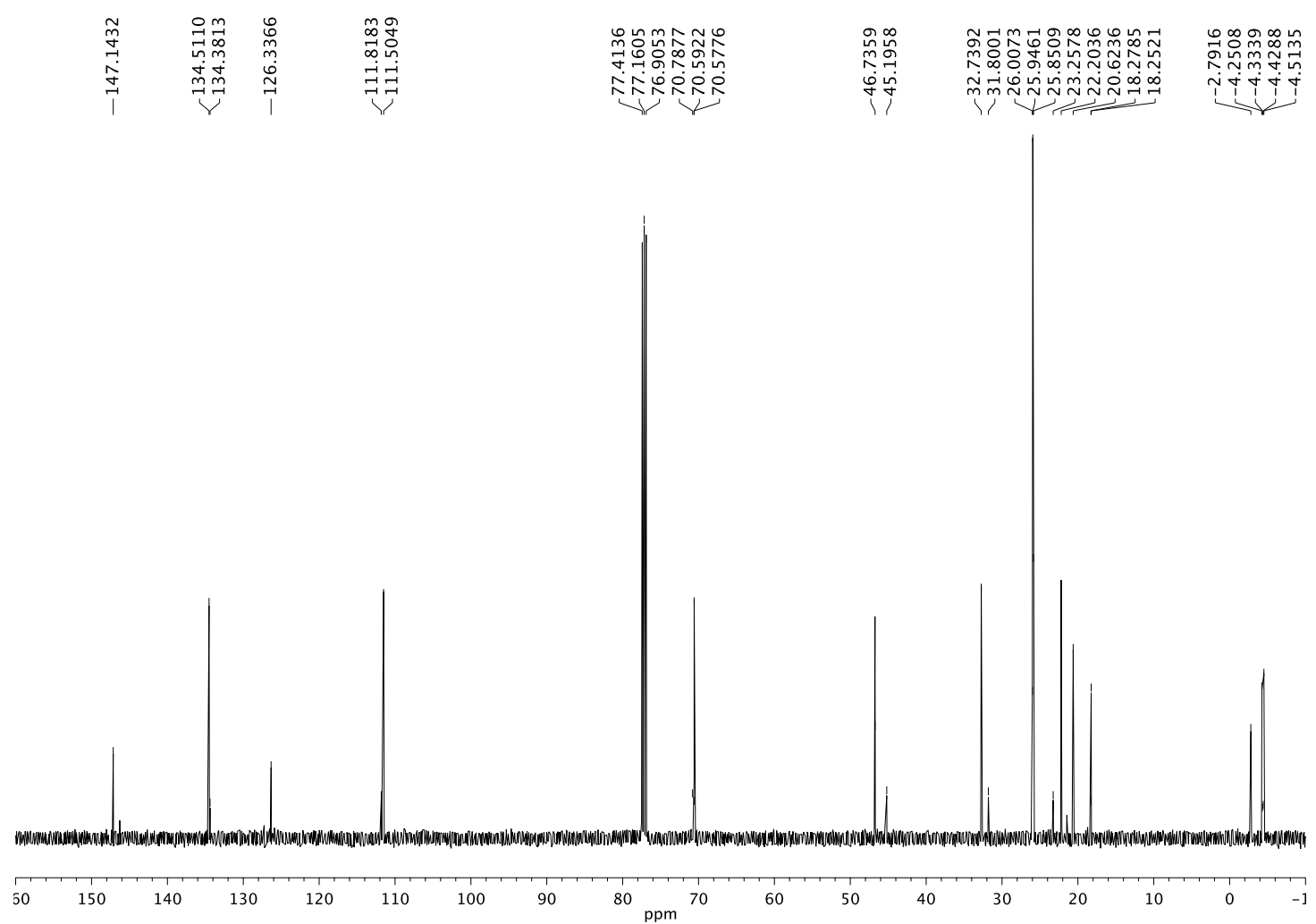

${ }^{13} \mathrm{C}$ NMR $\left(126 \mathrm{MHz}, \mathrm{CDCl}_{3}\right)$ of compound 13. 


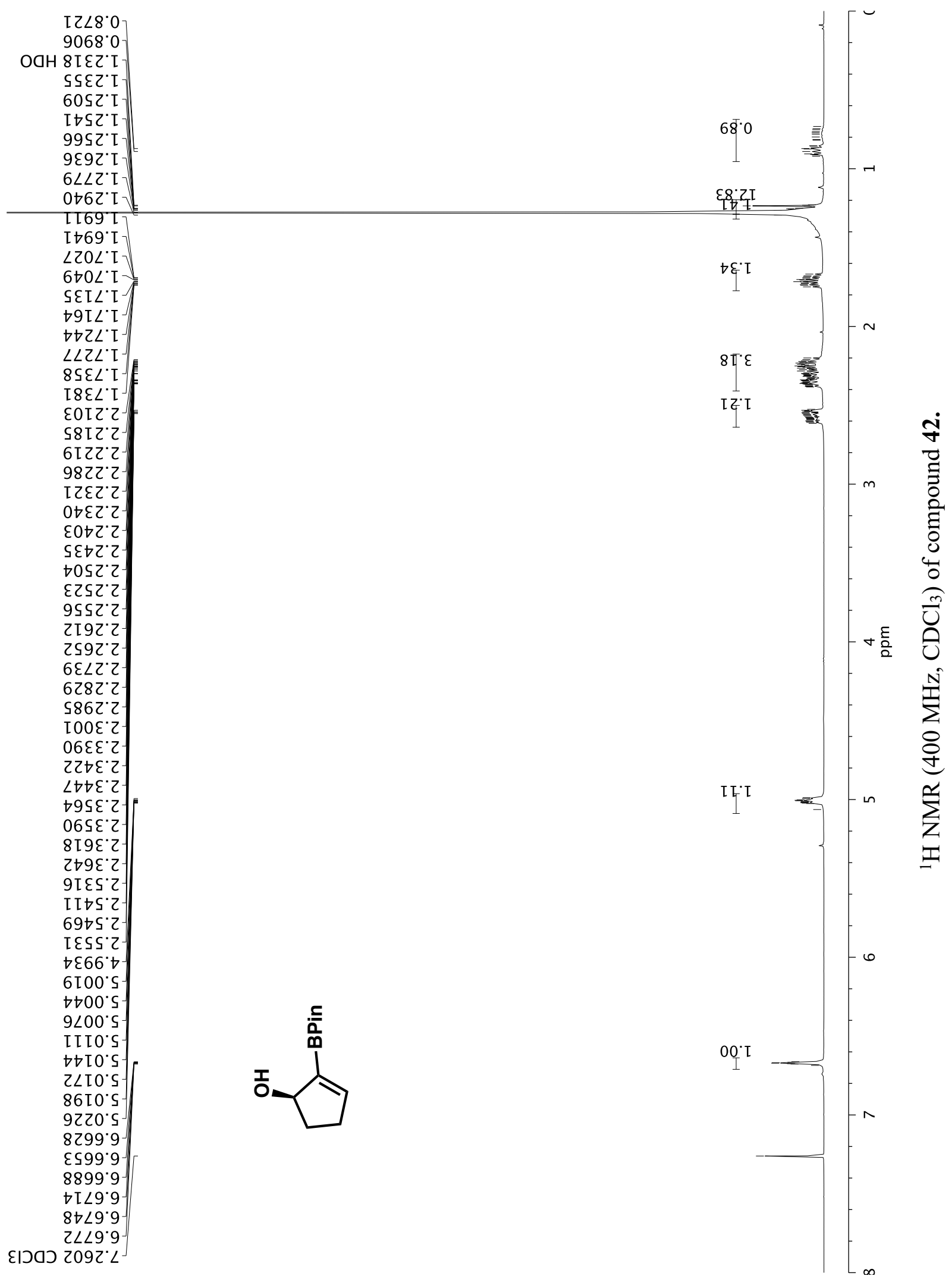




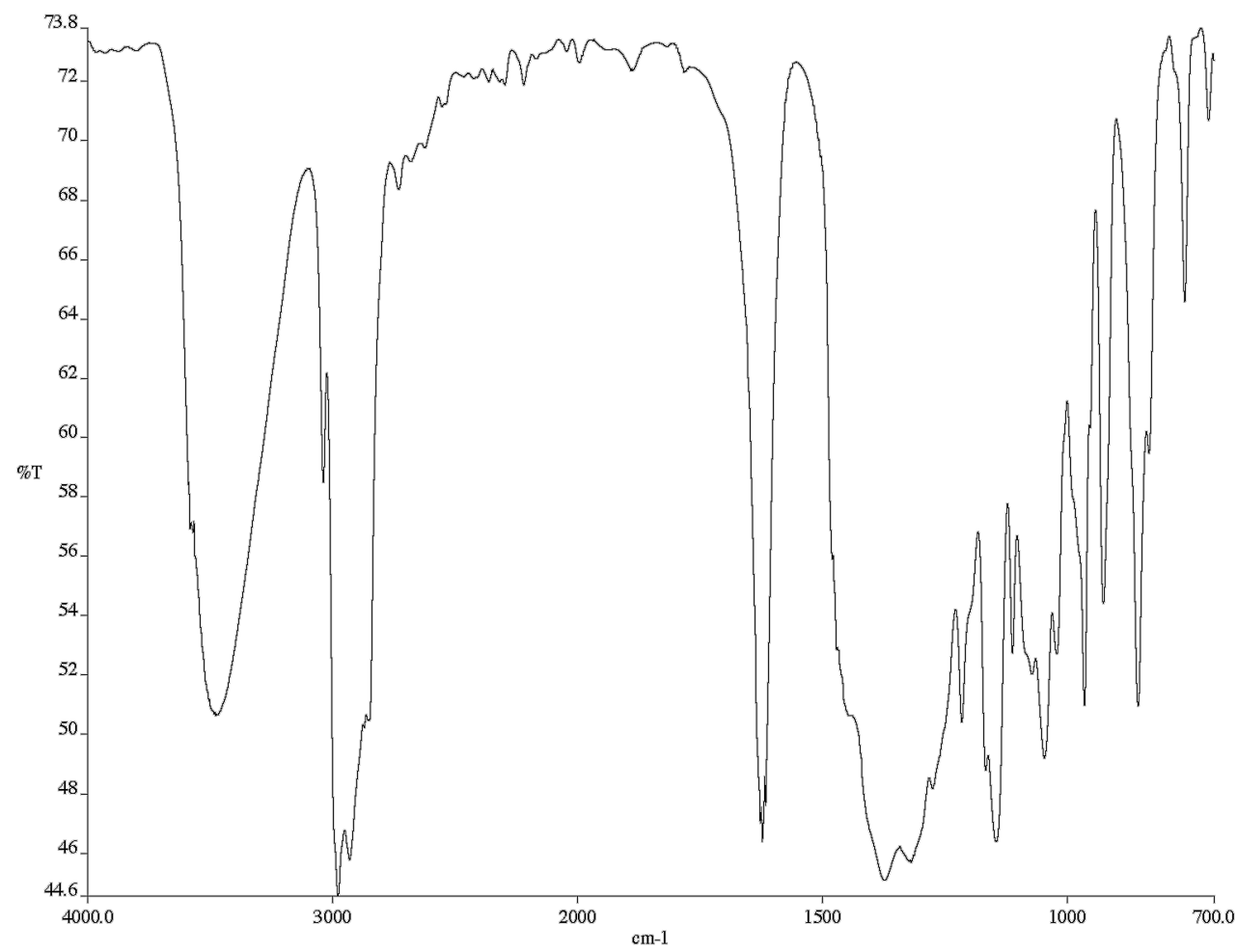

Infrared spectrum (Thin Film, NaCl) of compound 42.
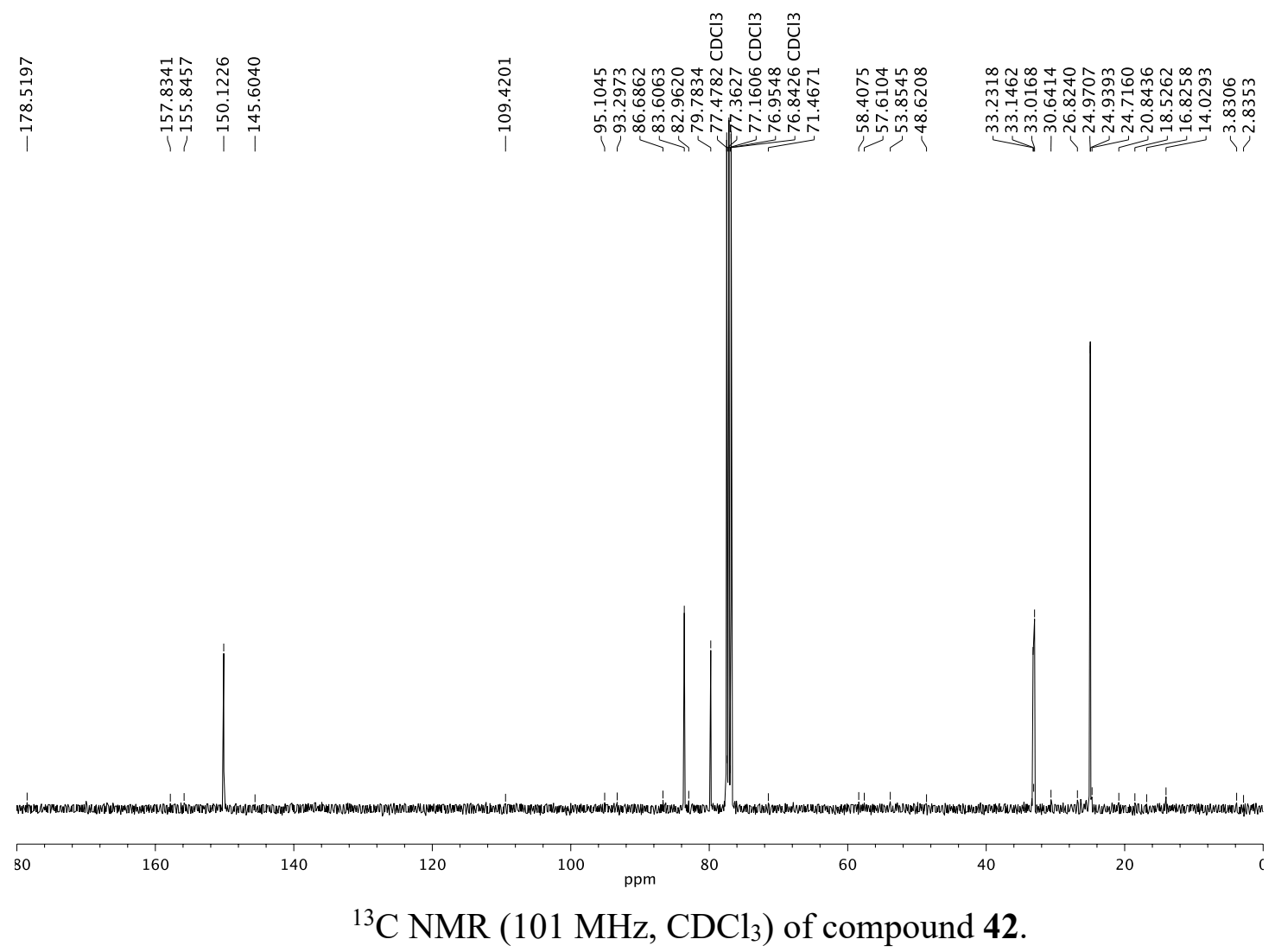


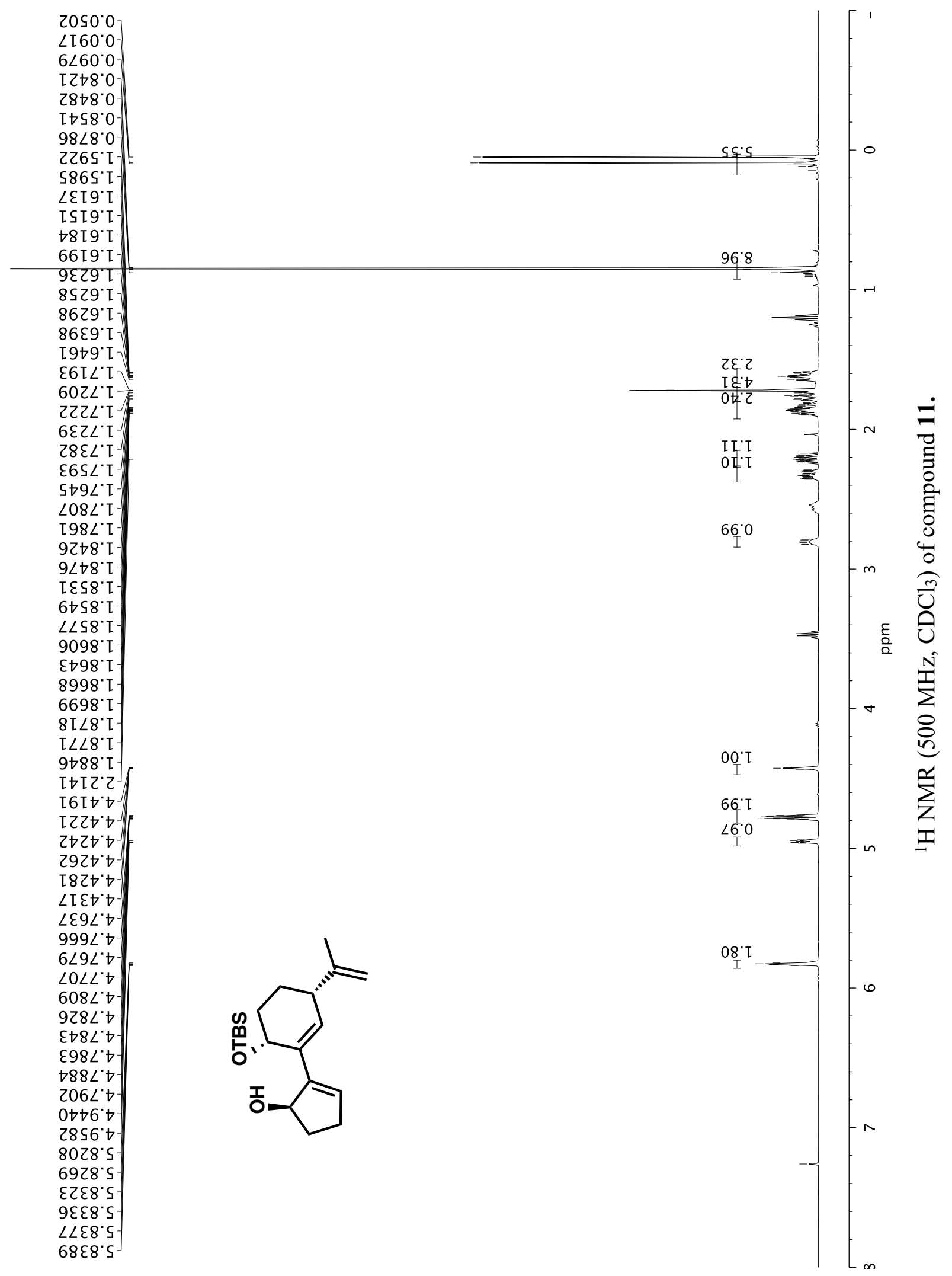




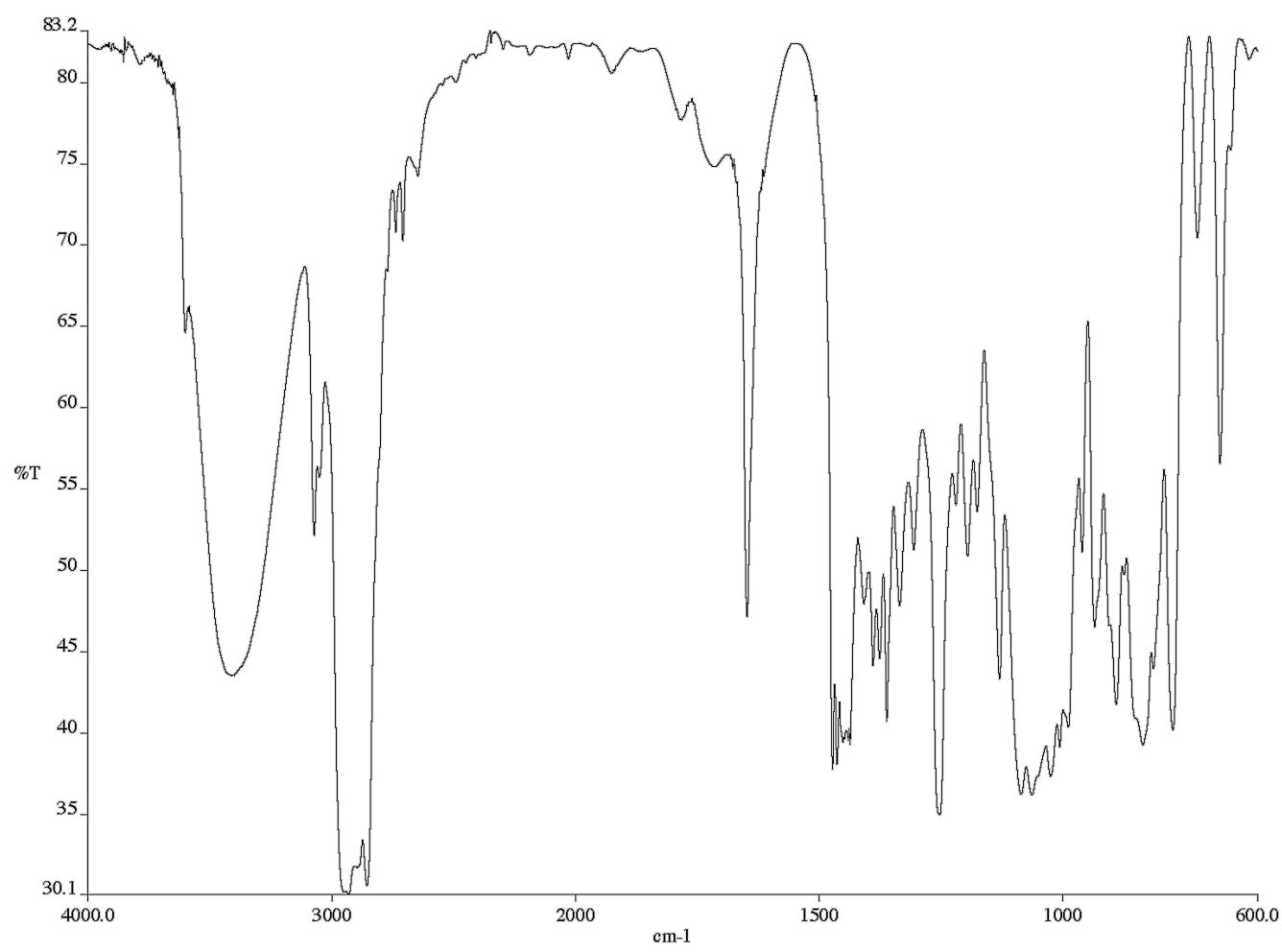

Infrared spectrum (Thin Film, NaCl) of compound 11.
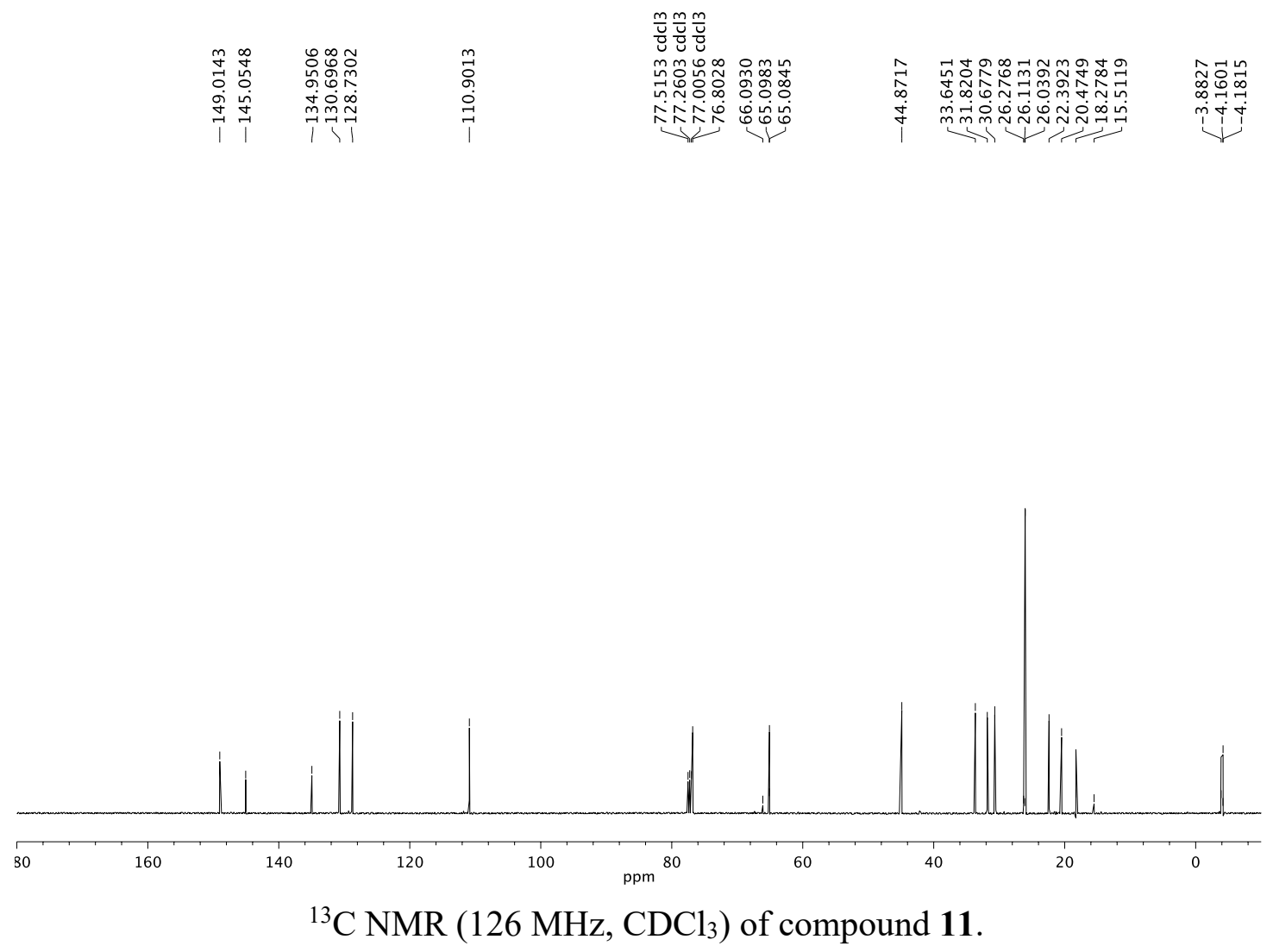


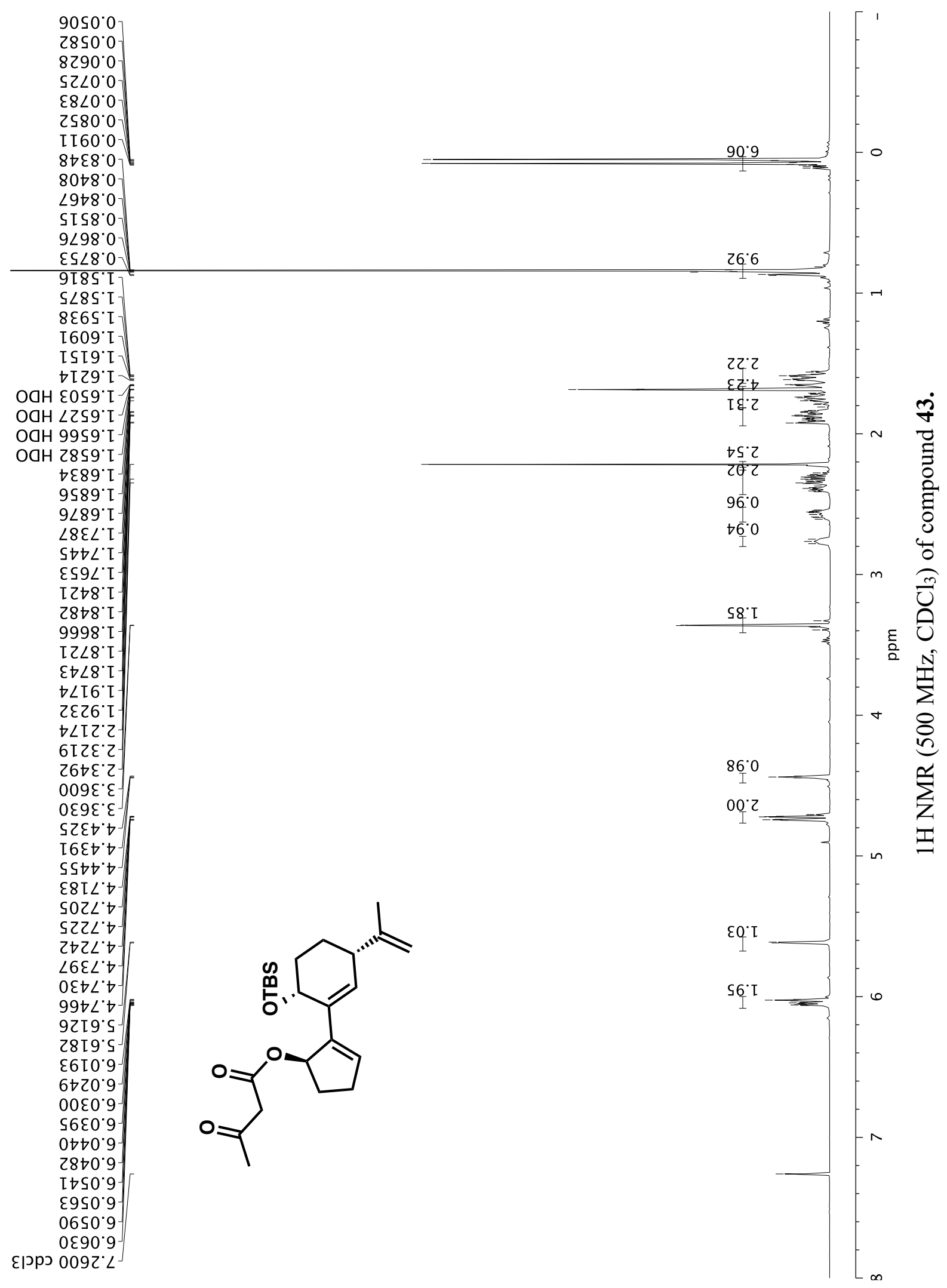




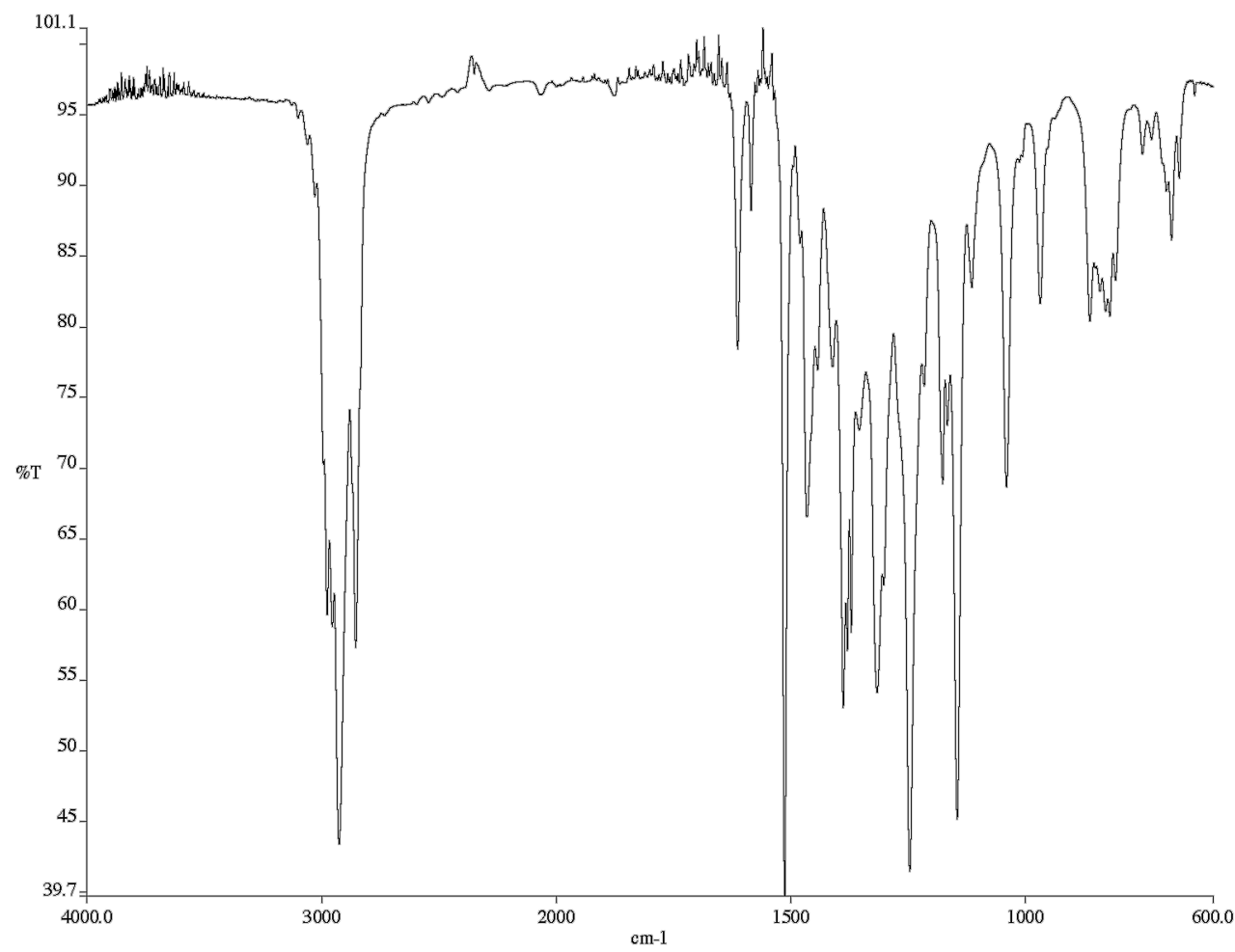

Infrared spectrum (Thin Film, $\mathrm{NaCl}$ ) of compound 43.
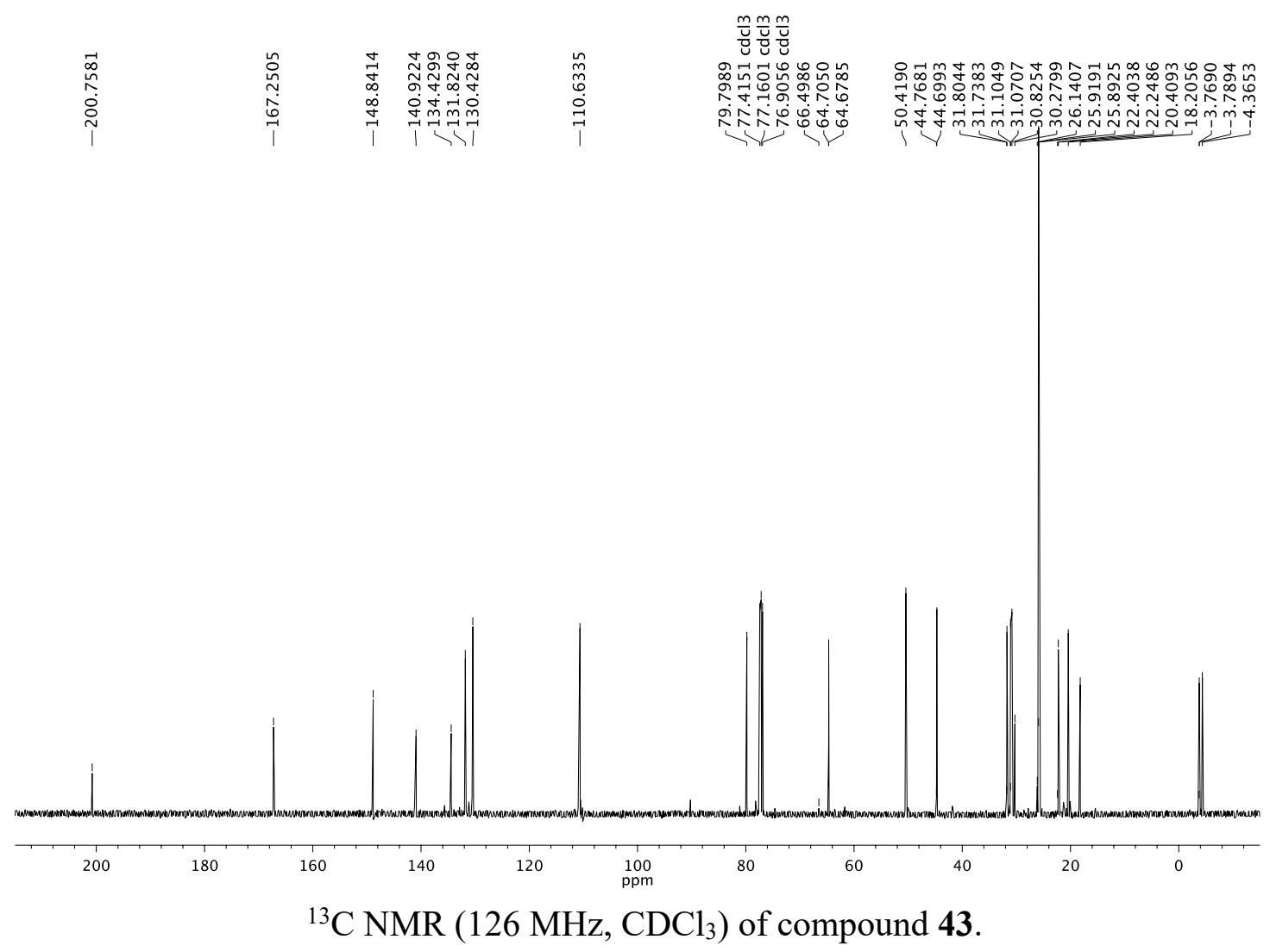


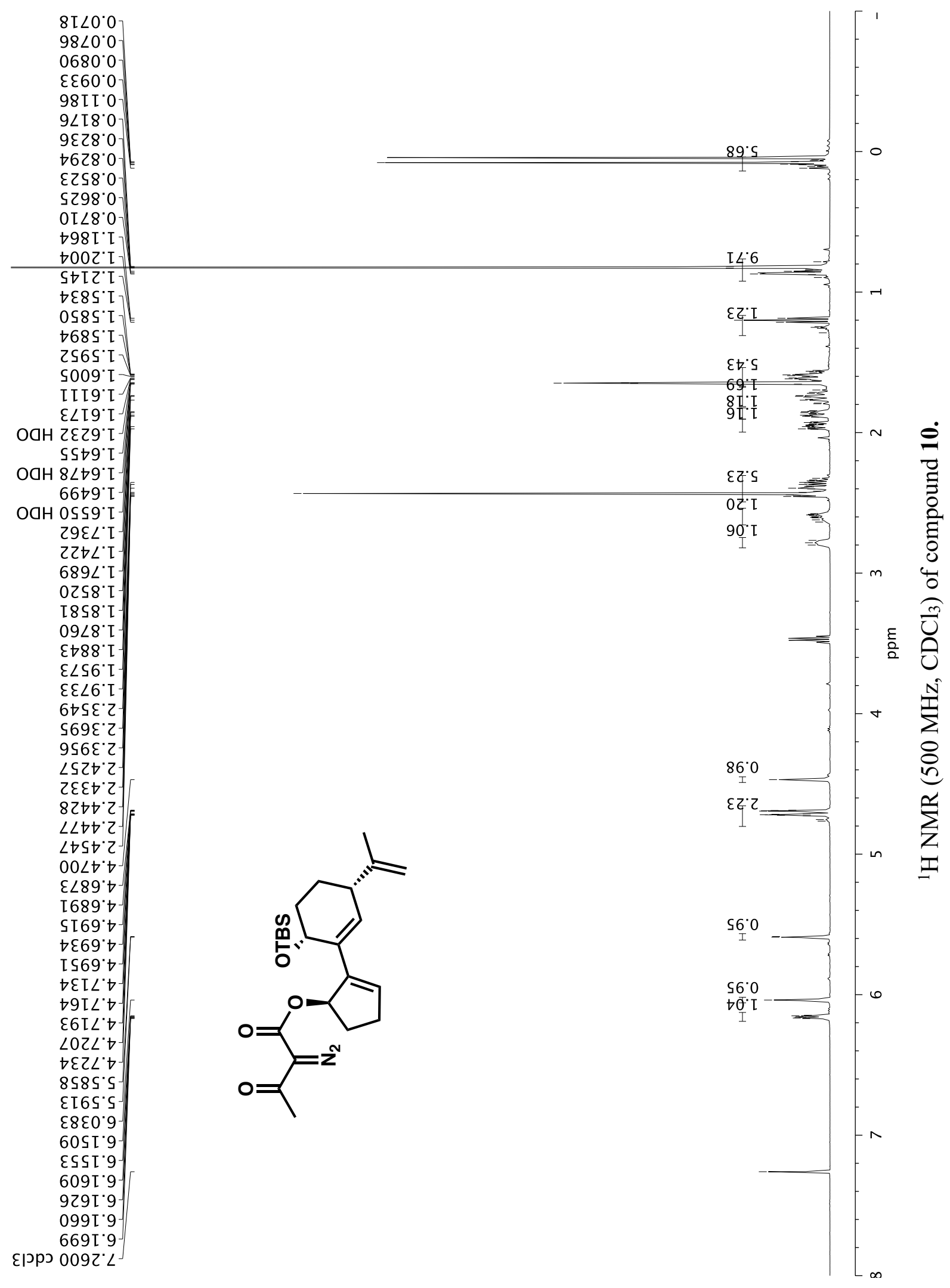



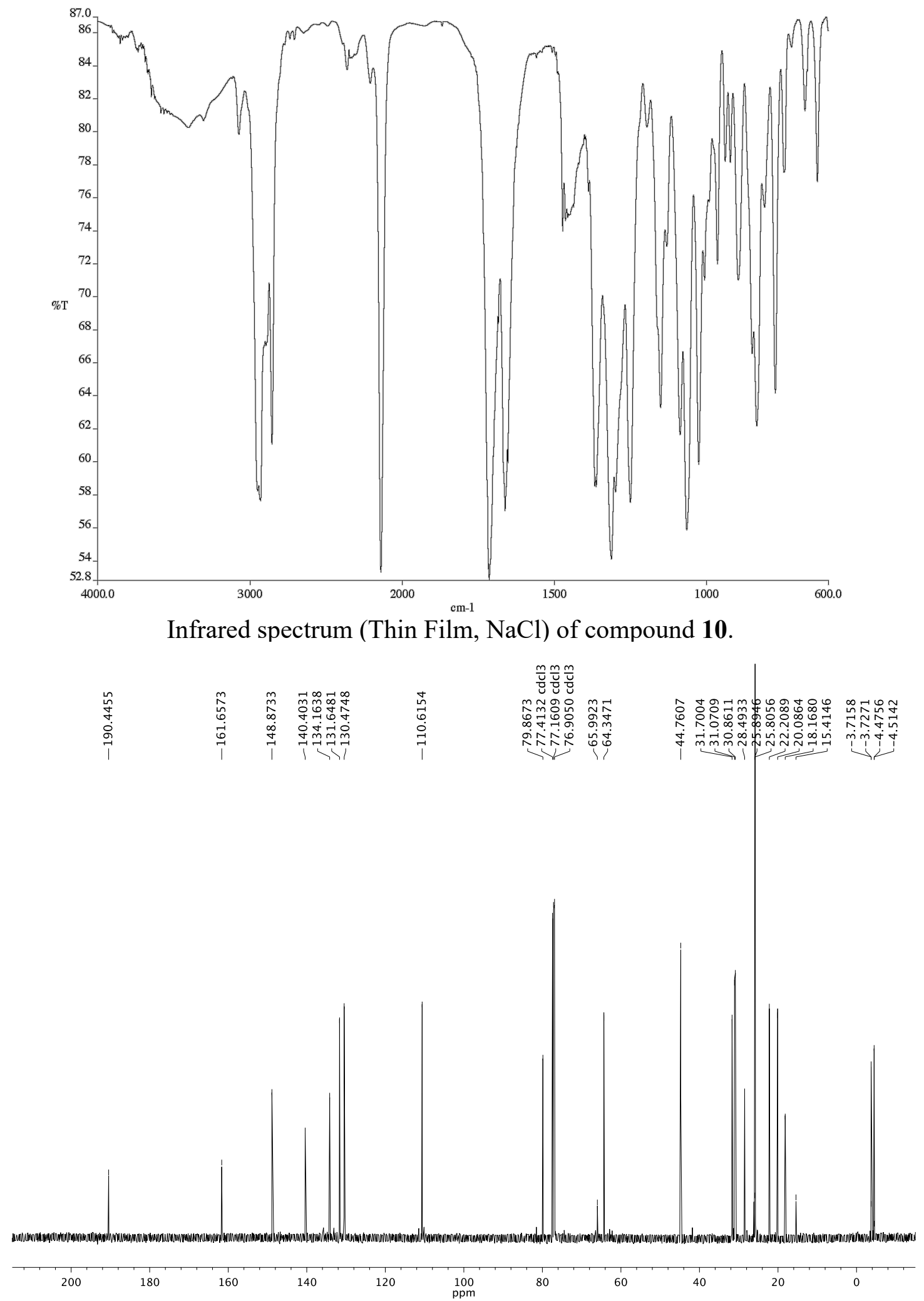

${ }^{13} \mathrm{C}$ NMR (126 MHz, $\left.\mathrm{CDCl}_{3}\right)$ of compound $\mathbf{1 0}$. 


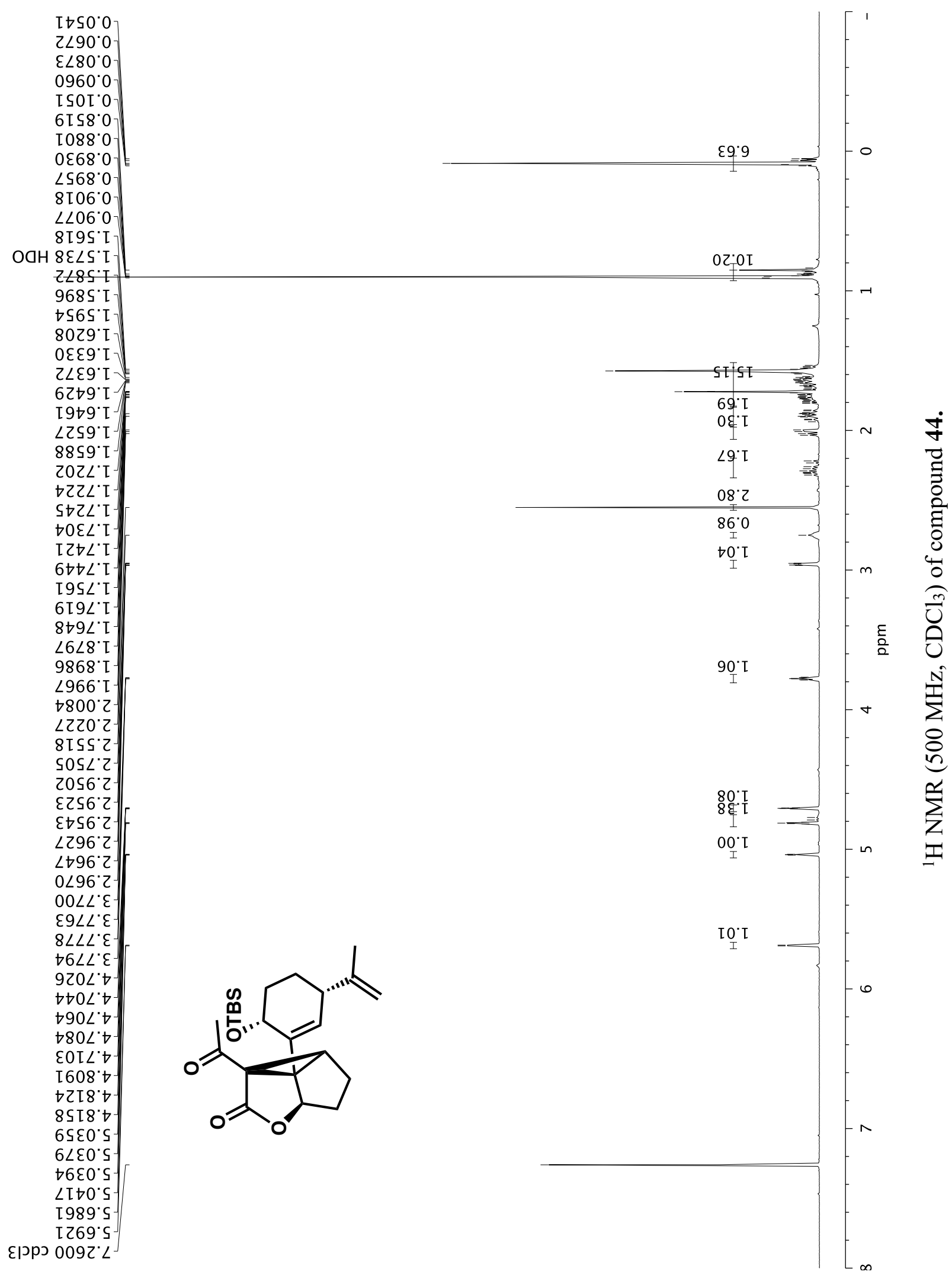



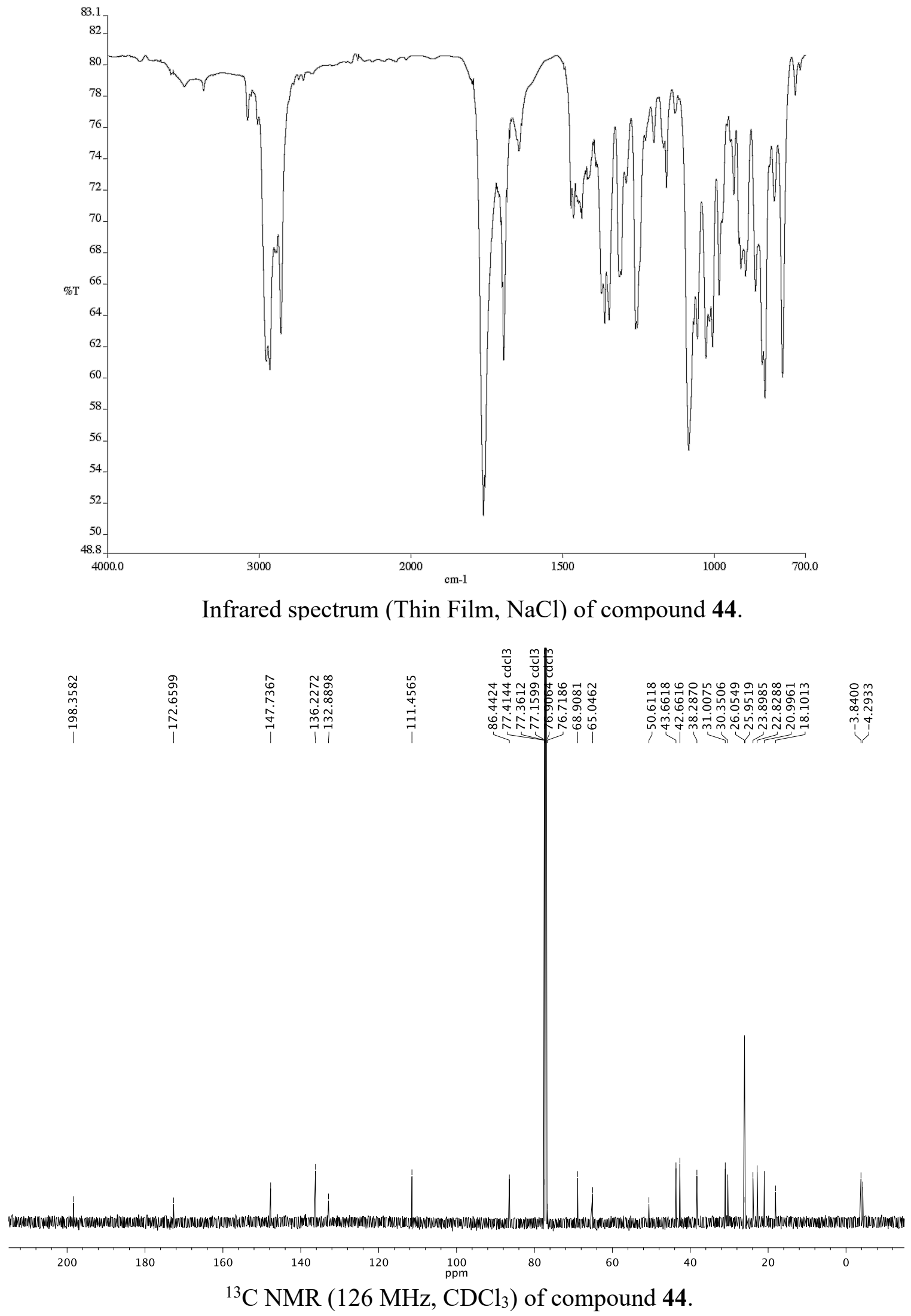


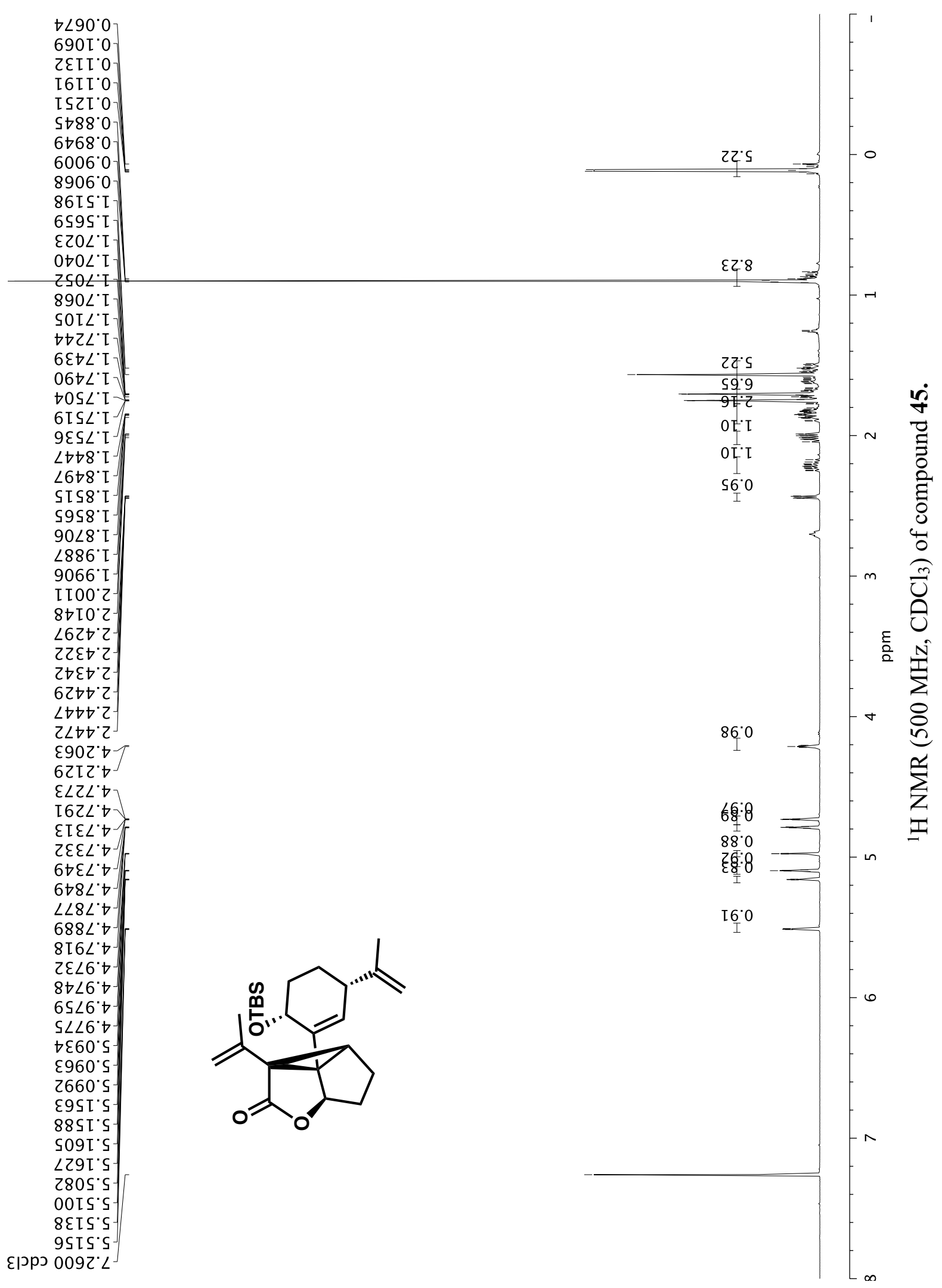




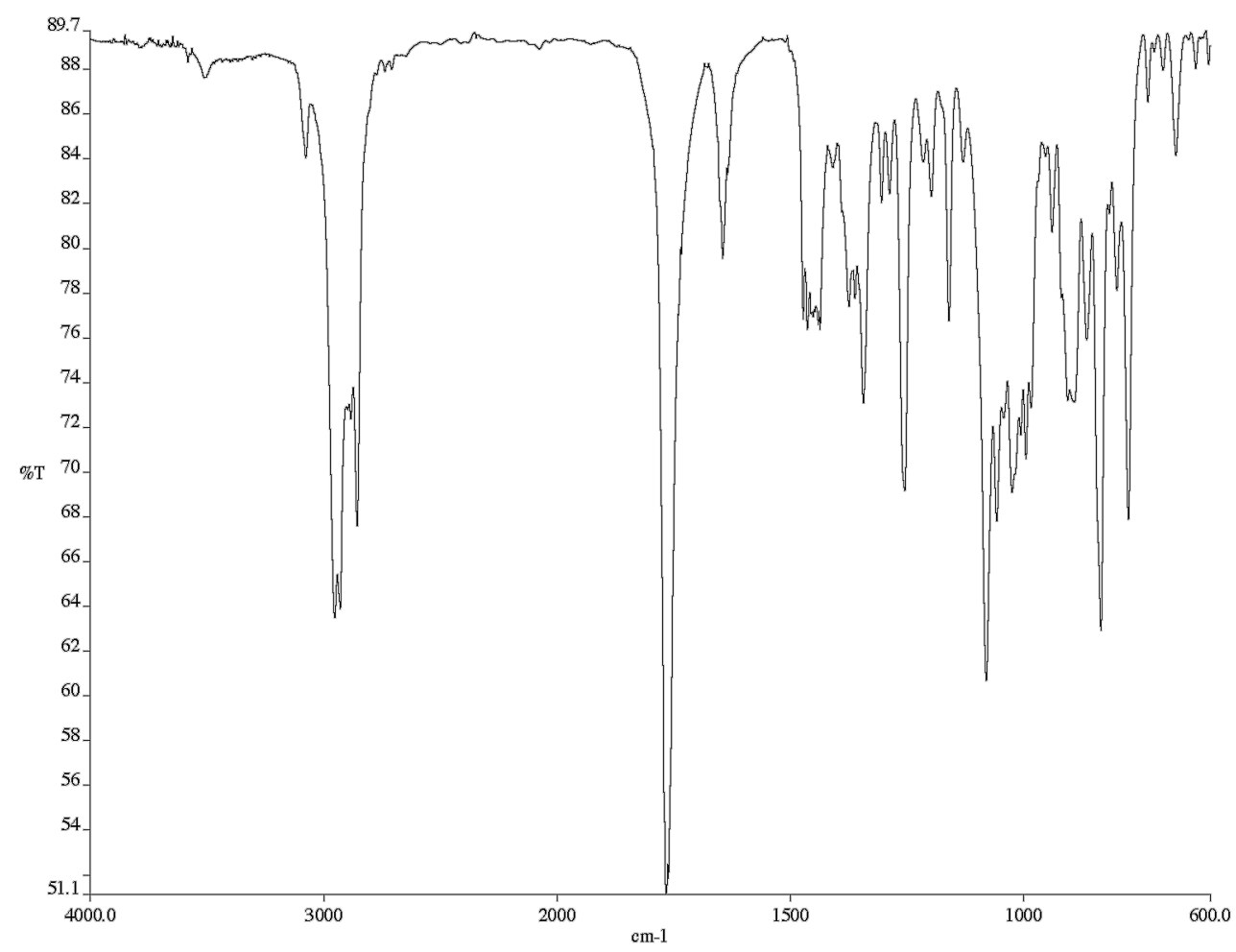

Infrared spectrum (Thin Film, NaCl) of compound 45.
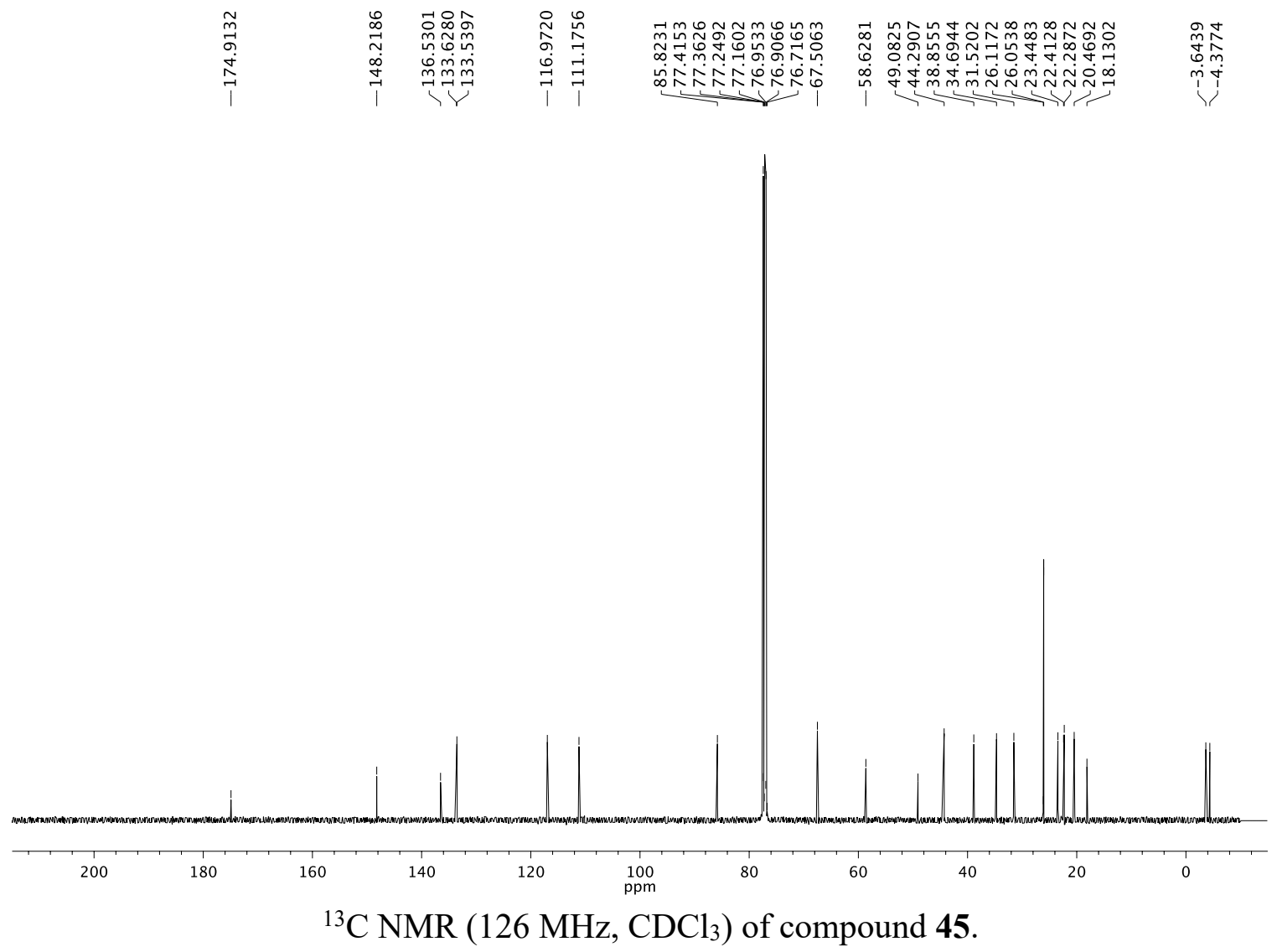


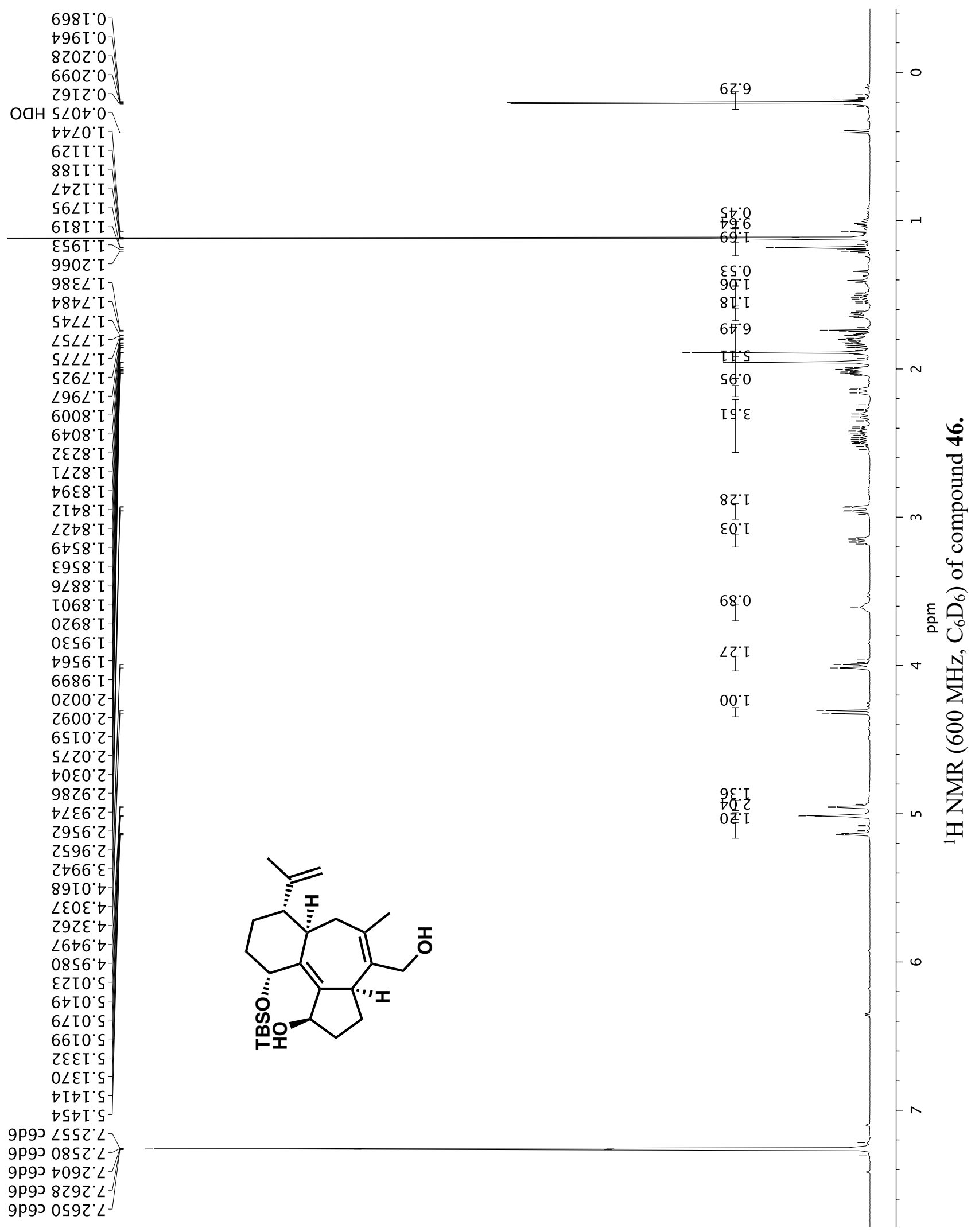



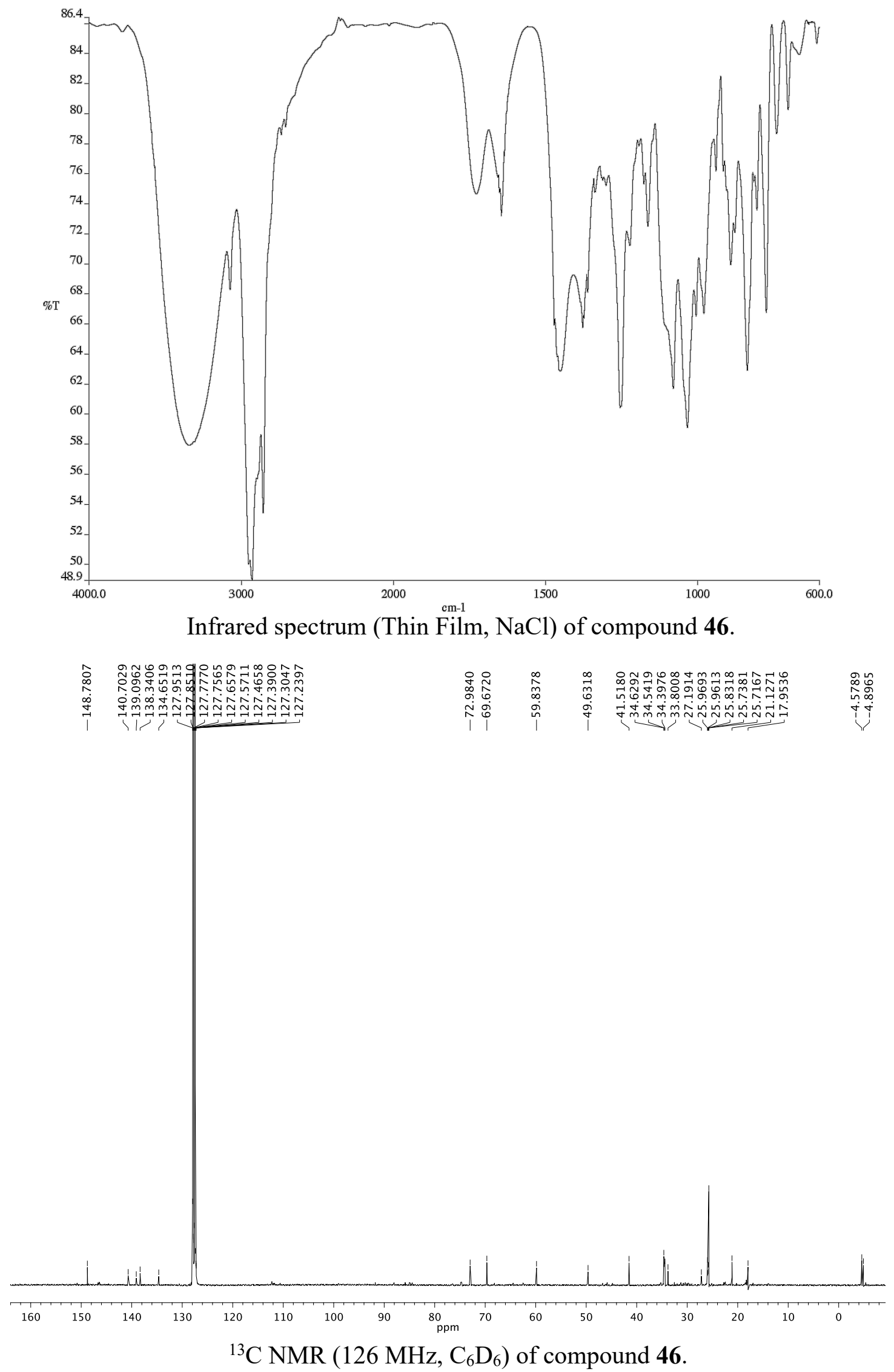


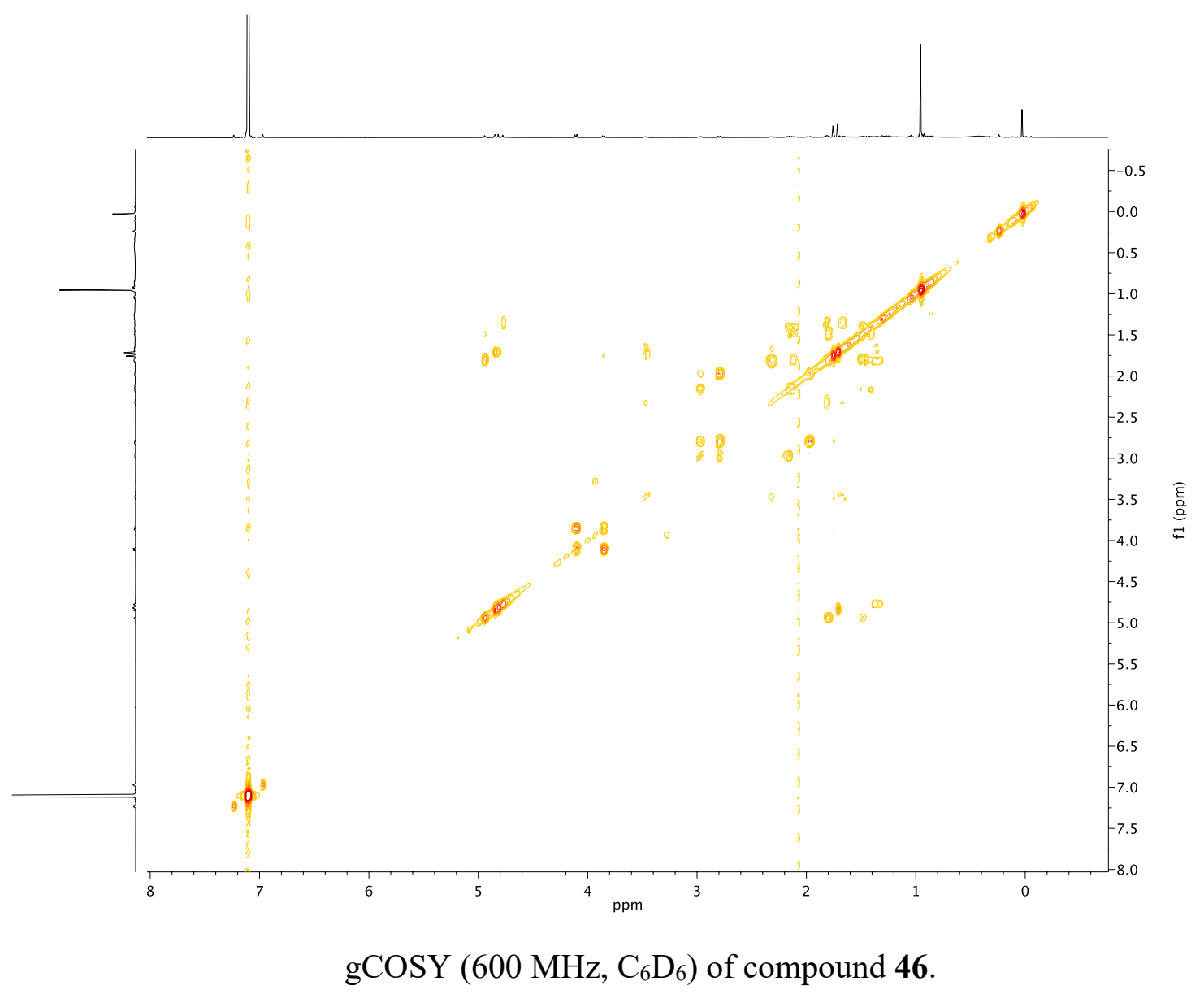




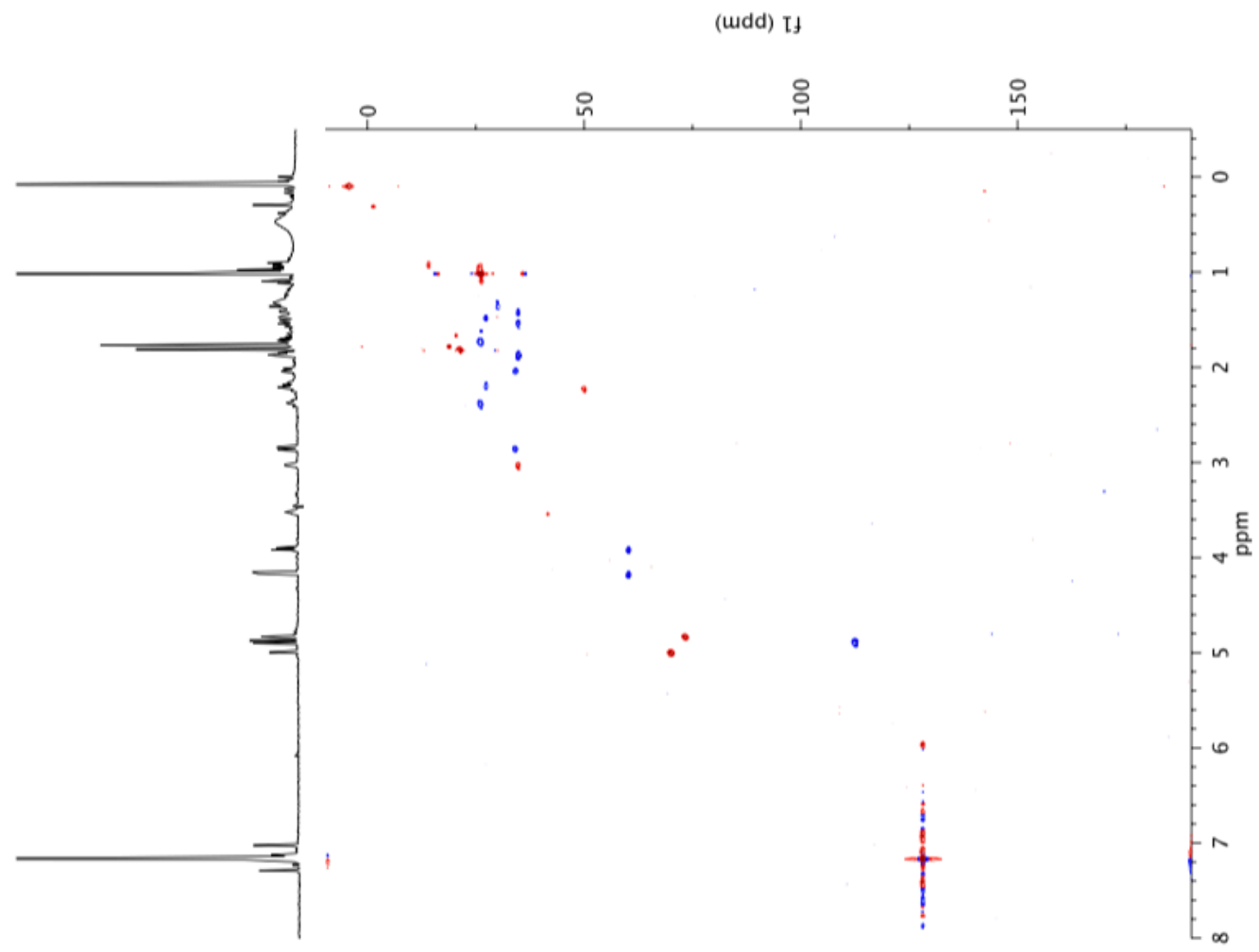

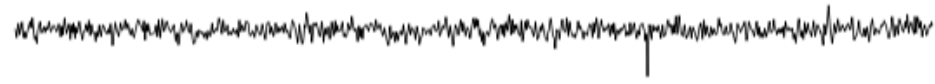

${ }^{1} \mathrm{H}-{ }^{13} \mathrm{C}$ HSQC (600 MHz, $\left.\mathrm{C}_{6} \mathrm{D}_{6}\right)$ of compound 46. 